\title{
Transmural care of terminal cancer patients : an evaluation study in the Eindhoven region
}

Citation for published version (APA):

Smeenk, F. J. W. M. (1998). Transmural care of terminal cancer patients : an evaluation study in the Eindhoven region. [Doctoral Thesis, Maastricht University]. Universiteit Maastricht. https://doi.org/10.26481/dis.19980416fs

Document status and date:

Published: 01/01/1998

DOI:

10.26481/dis.19980416fs

Document Version:

Publisher's PDF, also known as Version of record

\section{Please check the document version of this publication:}

- A submitted manuscript is the version of the article upon submission and before peer-review. There can be important differences between the submitted version and the official published version of record.

People interested in the research are advised to contact the author for the final version of the publication, or visit the DOI to the publisher's website.

- The final author version and the galley proof are versions of the publication after peer review.

- The final published version features the final layout of the paper including the volume, issue and page numbers.

Link to publication

\footnotetext{
General rights rights.

- You may freely distribute the URL identifying the publication in the public portal. please follow below link for the End User Agreement:

www.umlib.nl/taverne-license

Take down policy

If you believe that this document breaches copyright please contact us at:

repository@maastrichtuniversity.nl

providing details and we will investigate your claim.
}

Copyright and moral rights for the publications made accessible in the public portal are retained by the authors and/or other copyright owners and it is a condition of accessing publications that users recognise and abide by the legal requirements associated with these

- Users may download and print one copy of any publication from the public portal for the purpose of private study or research.

- You may not further distribute the material or use it for any profit-making activity or commercial gain

If the publication is distributed under the terms of Article $25 \mathrm{fa}$ of the Dutch Copyright Act, indicated by the "Taverne" license above, 


\section{Transmural Care of Terminal Cancer Patients}

An Evaluation Study in the Eindhoven Region 
The study presented in this thesis was conducted at the Research Institute HEALTH, which participates in The Netherlands School of Primary Care Research (CaRe), acknowledged in 1995 by the Royal Dutch Academy of Science (KNAW).

The study was supported by grants from The National Committee on Chronic Diseases (NCCZ), The Netherlands and The Scientific Fund of the Catharina Hospital, Eindhoven, The Netherlands.

TRANSMURAL CARE OF TERMINAL CANCER PATIENTS. An Evaluation Study in the Eindhoven Region /

Smeenk, Franciscus Wilhelmus Johannes Michaël. Thesis Maastricht. - With Ref. With summary in Dutch.

ISBN no. 90-9011503-X

Subject headings: Terminal Care / Home Care Services / Palliative Care / Neoplasms / Quality of Life / Shared Care / Integrated Care

(C) Elsevier/De Tijdstroom BV (parts of Chapter 1)

The BMJ Publishing Group (Chapter 2)

Elsevier Science Ltd. (Chapter 3)

Elsevier Scientific Publishers (Chapters 4 and 7)

Lippincott-Raven Publishers (Chapter 5)

Publication of this thesis was supported by ASTRA Pharmaceutica, The Netherlands

Book preparation: Unigraphic, Maastricht

Lay-out: Guy van Dael

Cover: 'De Ziekenoppas' (Carer of the Sick) by Jacob Vrel (1 $17^{\text {th }}$ Century Dutch painter) Printing: Unigraphic, Maastricht

Copyright (C) 1998 F.W.J.M. Smeenk

All rights reserved. No part of this publication may be reproduced, stored in a retrieval system, or transmitted in any form or by any means, mechanically, by photocopy, recording, or otherwise, without prior written permission of the author and the publishers of the published articles concerned. 


\title{
Transmural Care of Terminal Cancer Patients
}

\author{
An Evaluation Study in the Eindhoven Region
}

\section{PROEFSCHRIFT}

ter verkrijging van de graad van doctor aan de Universiteit Maastricht, op gezag van de Rector Magnificus, Prof.dr. A.C. Nieuwenhuijzen Kruseman, volgens het besluit van het College van Decanen, in het openbaar te verdedigen op donderdag 16 april 1998 om 16.00 uur

door

Franciscus Wilhelmus Johannes Michaël Smeenk 


\section{Promotor:}

Prof.dr. H.F.J.M. Crebolder

\section{Co-promotor:}

Dr. L.P. de Witte

\section{Beoordelingscommissie:}

Prof.dr. C. Spreeuwenberg (voorzitter)

Dr. J.P.M. Diederiks

Prof.dr. H.F.P. Hillen

Prof.dr. E. Schadé (Universiteit van Amsterdam)

Prof.dr. A.J.P. Schrijvers (Universiteit van Utrecht) 
'All the work of the professional team - the increasingly skilled symptom control, the supportive nursing, the social work, the home care, and the mobilization of community resources - are to enable the dying person to live until he dies, at his own maximum potential, performing to the limit of his physical activity and mental capacity with control and independence wherever possible. If he is recognized as the unique person he is and helped to live as part of his family and in other relationships, he can still reach out to his hopes and to what has deepest meaning for him and end his life with a sense of completion.'

\section{DAME CICELY SAUNDERS}

(In: Oxford Textbook of Palliative Medicine Doyle D. Hanks GWC, MacDonald N, Eds. Oxford: Oxford University Press, 1997;vii.)

Ter nagedachtenis aan mijn vader Voor Anneke, Michiel, Wouter en Anne Marije 


\section{Paranimfen:}

Dr.ir. Hein A. Fleuren, CFPIM

Drs. Harm Ch. Gooszen 


\section{CONTENTS}

CHAPTER 1

Terminal cancer: An introduction to the problem, study hypothesis

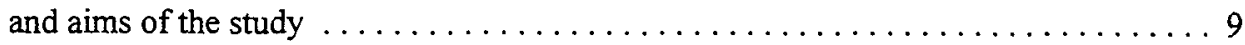

CHAPTER 2

The effectiveness of home care programmes for incurable cancer patients

on their quality of life and re-hospitalization. A systematic review $\ldots \ldots \ldots \ldots 33$

CHAPTER 3

Care process and satisfaction with care analysis of

a transmural home care programme $\ldots \ldots \ldots \ldots \ldots \ldots \ldots \ldots \ldots . \ldots . \ldots 49$

CHAPTER 4

Transmural care. A new approach to the care of terminal cancer patients:

Its effects on re-hospitalization and quality of life $\ldots \ldots \ldots \ldots \ldots \ldots \ldots \ldots$

CHAPTER 5

Transmural care of terminal cancer patients: Its effects on

the direct caregivers' quality of life $\ldots \ldots \ldots \ldots \ldots \ldots \ldots \ldots \ldots \ldots, \ldots \ldots$

CHAPTER 6

Does a transmural home care intervention programme improve

coordination and continuity of care? ........................... 103

CHAPTER 7

A cost analysis of transmural home care for terminal cancer patients

CHAPTER 8

Final considerations and future outlook

CHAPTER 9

Summary

CHAPTER 10

Samenvatting

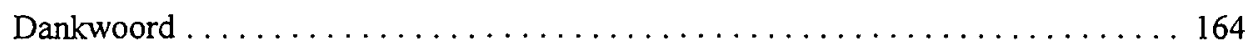

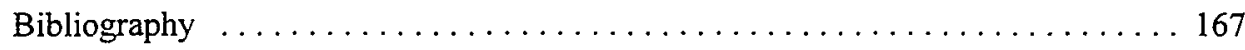

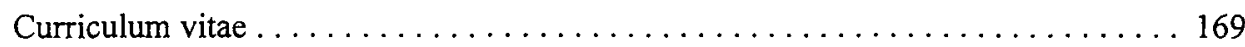




\section{CHAPTER}

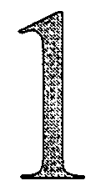

\section{Terminal cancer: An introduction to the problem, study hypothesis and aims of the study}

Parts of this chapter were published as:

Smeenk FWJM, van Haastregt JCM, Gubbels EMAC, Crebolder HFJM. Transmuraal zorgproject voor kankerpatiënten in Eindhoven. In: Handboek Thuiszorg. Schrijvers AJP, Stevens PGJJ, van Adrichem JAM, Crebolder HFJM, Duijnstee MSH, Sales HEM, Wiersema MI, eds. Utrecht; Elsevier/De Tijdstroom, 1997: D 7.1-1 - D 7.1-23. 


\section{Introduction}

The number of cancer patients is gradually increasing. ${ }^{1.2}$ Despite all the new treatment modalities, cancer remains incurable for more than $50 \%$ of cases. ${ }^{2,3}$ These incurable patients will inevitably enter a terminal phase of their disease. In this phase the patient's quality of life will diminish. ${ }^{4.5}$ Although most patients today wish to stay at home for as long as possible, ${ }^{6-8}$ the re-hospitalization rate of these patients remains high. ${ }^{9.10}$ This partially explains the high health care costs incurred by the terminally ill. ${ }^{11-13}$

If cancer patients cannot be cured, the main goal of any intervention used should be to maintain an optimal quality of life for as long as possible, rather than prolonging life regardless of its quality. ${ }^{14,15}$ Today, as a consequence of this accepted goal, quality of life is widely seen as one of the main outcome measures for intervention studies on this category of patients. ${ }^{15.18}$ Ideally, this care goal would be achieved by tailoring care to meet the individual needs of these patients.

This thesis describes the effects of a transmural home care intervention programme for terminal cancer patients, designed to improve coordination of care and thereby continuity of care as well, on:

- re-hospitalization of patients and place of death

- the patient's and their direct caregiver's* quality of life

- coordination of care, continuity of care and satisfaction with care

- health care costs

The study was performed in Eindhoven, The Netherlands in 1994. The intervention programme was based at Catharina Hospital Eindhoven.

In this introductory chapter we will give an overview of the problematic issues surrounding (terminal) cancer care, the study hypothesis, its design and aims.

\section{Epidemiology}

In 1993 , the crude incidence of cancer in The Netherlands was 417 per 100,000 for males and 376 for females. In total 60,500 newly diagnosed invasive tumours were registered, of which 5,000 had developed a second primary. During this period 36,500 patients died from cancer. After cardiac and vascular disease, cancer forms the second major cause of death. In The Netherlands 1 in 3 people will develop cancer at some stage in their life. ${ }^{19.20}$ The incidence of cancer is expected to gradually rise in the near future, mainly due to demographic changes in the population. ${ }^{2}$

In the South-eastern region of The Netherlands (i.e. where the Catharina Hospital is located) the incidence of cancer in the period from 1988 till 1992 was 454 per 100,000 person years in males and 306 in females (European Standardized Rate values; the Dutch population resembles the European Standardized population). ${ }^{21}$ Mortality in the same region during this period was 296 and 149 per 100,000 per year for males and females respectively. When one considers that the Catharina Hospital has an 'adherent' 
population of approximately 160,000 people, an estimated 356 terminal cancer patients can be expected each year in this 'adherent' population.

\section{Phases in cancer}

Dependent on the goal of treatment, and medical options available, several phases during the course of a patient's illness may be distinguished. ${ }^{22-24}$

The first is the diagnostic phase. In this phase a problem is identified, usually by the patient himself, who experiences complaints for which he seeks help. Sometimes, the disease is discovered coincidently or during routine 'screening'. The second phase is one of clarification and gathering information. In this phase a definite diagnosis is established. Next follows the phase of curative or palliative treatment.

When aiming for a cure it is sometimes necessary to give treatments that induce serious side-effects. This may have severe physical and psycho-social consequences for patients. ${ }^{25.28}$

When cure of the disease is, or has become impossible (sometimes after a symptom free interval), the patient will enter the palliative phase of the disease. In this phase, sooner or later, medical treatment is usually started to improve the patient's quality of life. Attempts are made to maintain the best quality of life possible, and for as long as possible. Treatment modalities in this phase are mainly symptom relief and complication reduction/treatment. Furthermore, (psychological) support in dealing with the disease is often necessary.

As the disease progresses complaints and symptoms often increase, quality of life declines and the need for care increases. In many cases deterioration suddenly accelerates, on average 3 months before death. ${ }^{4,529}$ This phase is named the terminal phase.

\section{The terminal phase}

In the terminal phase many problems may arise for cancer patients, which may substantially affect their quality of life, as well as that of their direct caregivers. ${ }^{40-33}$ Clinically, this phase will follow a more or less identical course for all the various categories of cancer (categorised according to the primary site). It has therefore also been named 'the common clinical terminal pathway', 3.5 During this phase, the clinical picture will mainly be determined by metastases, general physical symptoms as well as the patient's functional and psychological status.

Problems encountered by terminally ill cancer patients during their illness can affect their physical, psychological, and/or social quality of life. Many physical symptoms may arise. Addington-Hall and McCarthy found that in the last year of life $88 \%$ of all cancer patients reported being in pain; of these patients $66 \%$ were said to have found this very distressing. Other frequently reported symptoms were loss of appetite, constipation, dry 
mouth or thirst, vomiting and breathlessness. ${ }^{32}$ Others have also found a high prevalence of these symptoms in such patients. ${ }^{34,35}$

Psychologically, terminally ill cancer patients may experience a range of responses to their illness including denial, distress, anxiety, worry, anger, and depression. Feelings of helplessness, uncertainty and fear are also common. ${ }^{36,37}$ The prevalence of depressive symptoms is twice as high as those found in a general medical patient population (20$74 \%$ vs. $12-30 \%)^{37}$

Socially, this disease may disrupt normal daily routines. Tasks of everyday life such as cooking, shopping or even routine personal care may become impossible. ${ }^{38.39} \mathrm{Com}-$ munity social services may need to be employed in order to care for the patient at home. Social activities may diminish as a result of the disease, resulting in a loss of social contacts. ${ }^{40-42}$ The disease may also have a negative effect on family life. ${ }^{43}$

In a Dutch investigation, $75 \%$ of terminally ill patients had physical -, $88 \%$ psychological -, $13 \%$ relational -, and $25 \%$ financial problems. Thirteen percent also had problems with the care they received. The standard care available from agencies in The Netherlands failed to solve a high percentage (45\%) of these problems. ${ }^{44}$

Despite such problems, terminally ill patients generally still prefer to stay at home in their own intimate environment for as long as possible, and in most cases also prefer to die there. ${ }^{7.8,45}$ In contrast to this general wish by patients, a high re-hospitalization rate is observed. Furthermore, most of these patients continue to die in hospitals or hospices, and not at home. ${ }^{45-47}$ This partly explains the high health care costs incurred by these patients in their last year of life. ${ }^{11,13}$ The main reasons for this high re-hospitalization rate are: the inability to palliate disease symptoms at home, overburdened direct caregivers which may result in 'social re-admissions' to hospital, and badly coordinated care leading to poor continuity of care..$^{35.48-51}$

At this stage of the disease the main goal for all participating caregivers must be the same as that in the palliative phase i.e. maintaining the best possible quality of life for as long as possible, rather than aiming to prolong survival regardless of its quality. ${ }^{14.15} \mathrm{Care}$ must be tailored to meet the patient's individual needs. Independency of patients must be maintained for as long as possible. Ideally, and wherever possible, this care should be given to patients within their own homes.

During this phase the patient's problems are mainly multiple and vary in origin requiring the involvement of many caregivers from various specialties. It has been shown that the coordination of care is often poor, especially when more caregivers from differing echelons become involved. ${ }^{52.59}$ This may have a negative affect on the continuity of care, hereby leading to inadequate care, needless re-hospitalizations and a negative effect on the patient's quality of life. ${ }^{31,49,50,60,61}$ Continuity of care is defined by most investigators as 'the degree to which professional and informal care is provided as a coordinated and uninterrupted sequence of activities, in accordance with the actual needs of the patient during the disease trajectory' ${ }^{54.61-63}$ Continuity of care can thus be seen as a dimension of the care process itself. The strategy which caregivers apply to enhance continuity of care, is regarded as the coordination of care. ${ }^{52,54,64-66}$ Examples of 
coordination of care are the installation of a case manager/coordinator, the development of multidisciplinary care protocols and the installation of regular team meetings.

To overcome the problems in caring for terminally ill cancer patients, interventions have been set up to improve the care provided to patients. It was hoped that these intervention programmes would lead to less re-hospitalizations and a better quality of life for these patients. By lowering the re-hospitalization rate, it is feasible that health care costs will also be lowered.

\section{Interventions intending to solve the problems of caring for termi- nally ill cancer patients}

\section{Hospice care}

One approach to overcome these problems is that of hospices. They were founded in the United Kingdom and the United States of America. They became prominent as a result of Cicely Saunders' work. After working at St Joseph's Hospice in Hackney, she started her own free-standing 'St. Christopher's Hospice' in Sydenham in 1963.67 The care philosophy of the hospice movement reflects late-twentieth century western cultural and social values. It confronts the dying process openly, prepares people for and helps them accept death as an inevitable, natural phase in the life cycle. Hospices stress the importance of personal autonomy and, by implication, are critical of the perceived paternalism and authoritarianism within the medical profession. ${ }^{68}$ However, the effects of these hospices on the quality of life of terminal cancer patients, and re-hospitalization, remain unclear. The results of the large, multi-centre, quasi-experimental National Hospice Study in the USA, showed less utilization of aggressive interventional therapy and diagnostic testing in hospice patients, compared to similar patients receiving conventional terminal care. However, no significant effects on quality of life were observed. Satisfaction with care was higher for the direct caregivers, but not for the patients themselves. Patient re-admissions to hospitals, and costs, were significantly lower. ${ }^{68}$ Kane and co-workers, ${ }^{69}$ found in their randomized trial on hospice care, no significant effects on the quality of life of patients, re-hospitalization or costs.

\section{Home care programmes for terminally ill cancer patients}

Later, home care programmes began to materialize for these patients. These programmes were either aimed at improving symptom relief (e.g. pain control, psychological support or home nutrition interventions) or at improving the health care organizational structure in general.

- Studies of symptom relief at home

Results of those studies aimed at improving pain control show that most interventions have favourable results. ${ }^{70-74}$

Trijsburg and co-workers reviewed the effectiveness of psychological treatment interventions in cancer patients. ${ }^{75}$ They found that tailored counselling was effective 
with respect to distress, self-concept, (health) locus of control, fatigue, and sexual problems. Structured counselling showed positive effects with regard to depression and distress. Behavioural interventions and hypnosis were effective for specific symptoms.

Nutrition intervention studies showed that the outcomes of the home enteral nutrition interventions are questionable, possibly due to the short durations of intervention. ${ }^{76}$ The results of the home parenteral nutrition intervention studies showed that this technique is viable at home, has low complication rates, and probably has a positive effect on the patients' quality of life..$^{77.78}$

- Studies of home care programmes aimed at improving the health care organizational structure (especially coordination and continuity of care)

In general, results of home care intervention programmes aimed at improving the health care organizational structure do not show consistent results. The randomised study by Zimmer and co-workers ${ }^{79}$ did not show significant effects on the patient's quality of life nor re-hospitalization, whereas other studies have reported significant effects on these outcome measures. ${ }^{80,81}$

\section{Today's health care organizational structure in The Netherlands ${ }^{82}$}

In The Netherlands health care is characterized by its strong emphasis on community care. Primary care consists of general practitioners whose service is available 24 hours a day, community nurses (also available for 24 hours where necessary; i.e. 'intensive' community nursing), 'home help' for domestic assistance, 'meals on wheels', physiotherapy and a medical aids service which can provide equipment such as special beds, (wheel-)chairs etc. The general practitioner functions as a 'gatekeeper' for other community and institutional services. The Dutch community and hospital health care services are highly accessible. Primarily due to compulsory national or private insurance fully covering these services, or requiring only limited contributions. Secondly, most people live within a relatively short distance from any hospital because of the high population density in The Netherlands (average density of 400 per square kilometre; range 100$1,000)$.

If patients cannot be cared for at home, and do not warrant hospital admission, they can be cared for in 'nursing homes' (similar to GP hospitals or small community hospitals as seen in the United Kingdom). These homes have general medical as well as nursing staff.

Due to this well structured health care system, which, in theory, provides adequate care for all patients, the government's policy has been not to stimulate the development of a 'hospice movement'. ${ }^{2.83}$ It is therefore not surprising that hospice care in The Netherlands remains scarce. ${ }^{84}$ 


\section{Transmural care in The Netherlands}

This well structured health care system may be appropriate for patients with acute and uncomplicated health problems, but may show deficits for patients with more complex and chronic health problems, such as terminal cancer patients. ${ }^{61}$ In The Netherlands terminally ill cancer patients also show a high frequency of re-hospitalization. ${ }^{9}$ In 1994 approximately 10 percent of all hospital admission days were for the care of cancer patients. This accounted for 11 percent of the hospitals' budgets (i.e. 1.073 million Dutch guilders; in 1994 one US dollar costs approximately 1.80 Dutch guilders) ${ }^{85}$

Some of these hospital (re-)admissions may have been due to poor coordination of care thereby preventing an optimum continuity of care. In The Netherlands, as in most other countries, there is no regular authority or person embedded in the health care structure, who is responsible for the coordination of care. ${ }^{82}$ Coordination of care seems to be especially poor when more caregivers are involved and when patients need care from hospital and primary care teams. ${ }^{49.50 .52 .60 .86}$ This is usually the case for most terminal cancer patients. All too often these different caregivers appear to perform their tasks individually rather than collaboratively. This may lead to needless investigations, (re-) admissions and inadequate care, which may consequently also have a negative affect on the patient's quality of life.

Special working parties ${ }^{2.83}$ who studied this problem concluded that caregivers from primary and hospital settings should better coordinate their care and cooperate in the tailoring of care to meet patients' individual needs. Also, those caregivers providing specific care should be able to supply the care required regardless of the patient being hospital- or community based. The complementary approach is hereby emphasized. This should be accomplished with mutual agreement, cooperation and shared responsibility, thereby breaking down the 'virtual walls' between primary and hospital care. This kind of care was named transmural care. Other terms often used in this context are 'shared care' or 'integrated care'. In time, several descriptions of transmural care were devised by various authorities. ${ }^{87}$ The National Council for Public Health Care's description is currently being adopted by most caregivers and authorities. Transmural care is described by this National Council as follows: 'Transmural care encompasses those kinds of care which are tailored to meet patient's needs. It is provided by caregivers from primary and hospital teams on the basis of coordination and cooperation, with shared responsibility and specification of delegated responsibilities. ${ }^{.88}$

In transmural care, improving coordination and thereby continuity of care, as well as efficiency and care tailored to meet patient's individual needs, are important goals to strive for. It is therefore not surprising that this kind of care has been highly recommended for development at a local level by national health care politicians. ${ }^{83,89,90}$ As a consequence, many transmural care projects have been introduced.

Persoon and co-workers ${ }^{91}$ found 353 operational transmural care projects between 1990 and 1996. Two hundred and thirty-five were organized within general health care, 76 within mental health care, 32 within care of the elderly, and 10 within care of the 
disabled services. Forty percent of the projects within general health care were of an individual community nurse or community care team character (consisting of community nurses, home helpers, and general practitioners). These primary caregivers cooperated closely with specialized hospital based caregivers. Eighteen percent of the projects only consisted of protocols. These protocols contained standardized written guidelines, mostly for specific patient categories, and were developed by carers from both primary and hospital settings. In mental health care $40 \%$ of the projects were of a case management character, and $13 \%$ of an intensive community psychiatric care character. In the elderly health care services, the majority of projects were of an out-reaching nursinghome character. The patients being cared for in these projects almost invariably had a chronic disease which may have been complicated by temporary exacerbations or acute complications, e.g. rheumatoid arthritis, cancer, diabetes mellitus and so on. However, transmural care programmes had also been developed for patients who required care for a short period of time, such as terminally ill patients, convalescent surgical patients, stroke patients et cetera. ${ }^{87}$ Caregivers frequently involved in these transmural projects in the general health care setting were community nurses/caregivers from home help services (87\%), medical specialists (86\%), hospital nurses $(77 \%)$, and general practitioners $(57 \%){ }^{91}$ In The Netherlands there were no extra financial resources available for these projects. Most of them were, and still are, financed from the institutions own budgets. Some were only temporarily financed as a project. Of the 235 projects in the general health care setting only 62 performed an evaluation study. Most of these studies, however, contain serious methodological deficiencies. Only 3 of them met the most basic methodological demands for measuring the effects of an intervention: i.e. contained a control group and performed both pre- and post-intervention measurements. Whether the aims of transmural care (efficiency and care tailored to meet patient's individual needs) had really been achieved or not remained unclear. Persoon and coworkers also noted that in order for political decisions to be made, concerning the issue of transmural care facilities being implemented on a permanent basis, scientific research meeting the standard quality criteria was urgently required.

\section{Development of the Eindhoven transmural care programme}

The development of this transmural home care intervention programme in our region was feasible due to general and local developments in social, political, economic, and professional areas.

The general developments included the government policy encouraging the further development of transmural care, patients wanting care better tailored to meet their actual needs, preserving their independence and enabling them to stay at home surrounded by their loved ones for as long as possible. The following items were also of significance:

- A desire to control ('terminal') health care costs. It was assumed that community care would be cheaper than hospital care. ${ }^{89}$

- Hospitals being faced with patients who, after receiving the medical treatment for 
which they were admitted, couldn't be discharged because they were still physically or psychologically too weak. Admission to a 'nursing home' would then be appropriate. However, this was generally impossible within an acceptable time frame, mainly due to long waiting lists. These patients, therefore, often remained in hospital, occupying beds intended for other patients needing hospital treatment. In The Netherlands this phenomenon is referred to as the 'blocked bed problem'. ${ }^{92}$

- Demographic developments, leading to an increasing chronically disabled ageing population. ${ }^{93}$

Local developments that have contributed to the development of this project were:

- The establishment of a multidisciplinary oncology ward in our hospital in 1993.

- Oncology being appointed as one of the hospital's main spearhead specialities.

- The local General Practitioners organization who expressed their wish for a better coordinated discharge of patients.

- The foundation of a working party by nursing and home help staff of hospital and community based teams. Their main aim was to improve the continuity of care.

From these developments rose a multidisciplinary working party which had to develop transmural care models for terminal cancer patients. A precondition was that these care models should not interfere with existing care structures. Representatives from primary (medical, nursing, home help, pharmacy, and local volunteer organizations) and hospital (medical, nursing and management) care teams were members of this working party. It received national recognition with a grant from the National Committee on Chronic Diseases (NCCZ). The working party received advise from a national supervision committee.

The working party aimed to develop a model of care better tailored to meet the patient's individual needs, and where every effort should be made to provide this care within the patient's own home. In order to be able to achieve these goals, an optimum coordination of care was needed.

Initially there was some delay in the development of the model because of reservations expressed by some general practitioners. They argued that they provided adequate care for these patients in the current setting, taking into account the availability of community nursing and home help. Furthermore, they were afraid that the hospital's real intention of starting this project was to start providing (hospital) care to patients at home, thereby overriding the general practitioners' responsibility for community care. Lastly, they felt that the project may well mean a lot of extra work for them without appropriate reimbursement settlements. Eventually an agreement was reached amongst all parties involved and the transmural care model was complete in the spring of 1993.

\section{The transmural home care intervention programme}

The transmural home care intervention programme studied in this thesis consists of 4 main elements: a specialist nurse coordinator, a 24-hour telephone service in the hospital with access to a transmural home team, a collaborative home care dossier (case file) and 
protocols designed for specific care. All these elements intended to improve the coordination - and continuity of care.

\section{The specialist nurse coordinator}

The specialist nurse coordinator was the key-person in this intervention programme. On request of the treating specialist, she prepared the necessary patient discharge arrangements. Patient's wishes and (care) needs were assessed by her as well as the possibilities for patient support by informal caregivers. Taking these findings into account she planned and arranged home support by professional caregivers, which would naturally be tailored to meet the patient's individual needs. In order to carry this out successfully she needed to have daily contact with a wide variety of caregivers, from medical specialists to home helpers. Therefore, this functionary needed to have both specialized nursing knowledge and experience, as well as excellent interpersonal social skills. She monitored the care provision process, tracked down and solved possible shortcomings. Others had already shown the positive effects a care coordinator can have on the care process. ${ }^{63.94 .96}$

\section{The 24-hour telephone service and transmural home team}

A 24-hour telephone service was installed on the hospital's multidisciplinary oncology ward. This service was manned by nurses from the ward. In order to do this, they were specially trained in giving assistance to patients by telephone. In case of problems arising at home this service can be contacted for advice by patients or their caregivers. It could be reached day and night by telephone via a direct line (no operator delays or interference). In case of medical questions a specialist consultant could be contacted using the service. In order to give adequate advise, a copy of the patient's home care dossier, containing all the essential medical and nursing information, was at hand.

If specific nursing problems could not be solved by the primary care team, support was provided by specialized nurses from the hospital's transmural home team on request of the patient's general practitioner. In general, problems which the team had to solve involved the high technology devices often used for treatment, such as special devices used for epidural analgesia or intravenous administration of drugs. The transmural home team consisted of nurses from the hospital's casualty and 'day care' departments. During on-call hours they could be contacted by semaphone. Whilst administering care in the patient's home they were legally insured (as if working in the hospital itself).

The organizational structure of the 24-hour service and transmural home team is depicted in Figure 1.

\section{The home care dossier}

The home care dossier was developed to improve communication between caregivers and thereby to enhance the coordination of care. ${ }^{97-99}$ Caregivers from primary and hospital care teams were asked to collaborate in reporting their findings and actions undertaken. Hereby all caregivers were informed about findings and care plans. The 
record consisted of the following items:

- An informed consent. This statement was included for reasons of privacy. By signing the informed consent the patient permitted other caregivers to read his or her file, to consult other caregivers, if necessary, about treatment and/or care and to document new medical or care information.

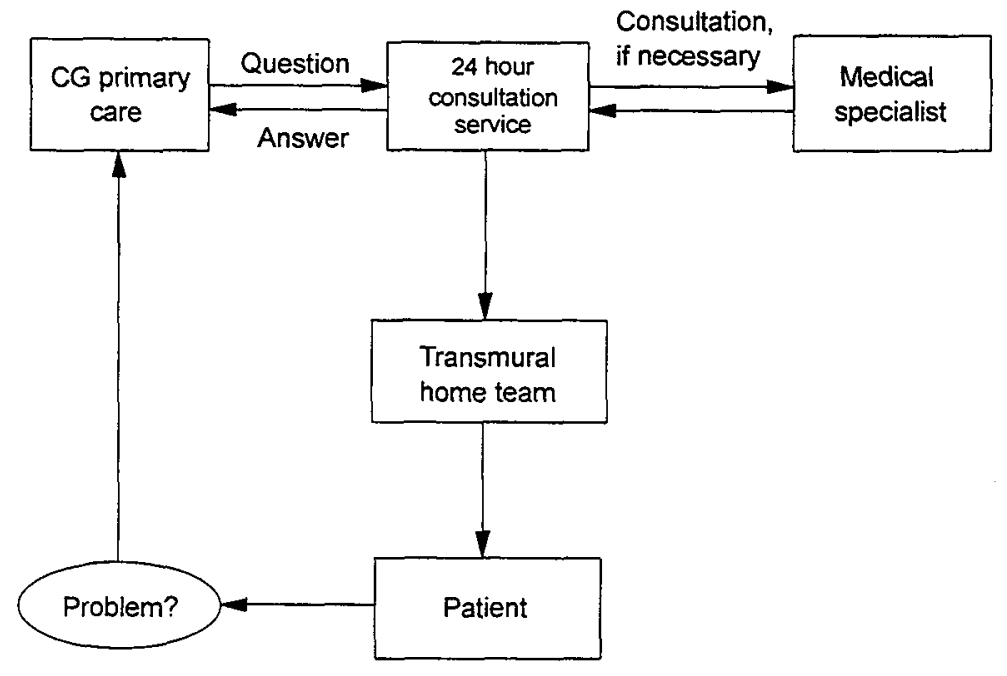

Figure 1 Organization structure of the 24-hour telephone service and the transmural home team ( $C G=$ Caregiver)

- A list of those caregivers involved. This list comprised the names, addresses and telephone numbers of all caregivers involved in the care of the patient.

- A preliminary discharge report for the general practitioner. Through this report the general practitioner (as well as other caregivers) were instantly informed of the most recent medical information.

- A nursing transfer report for the community nurse. This nursing report was written using a checklist for guidance. This checklist was used by both hospital and community nurses. Areas of patient health were assessed using a standard format, thus enhancing a consistent approach to care.

- A transfer report for home helpers. This report dealt with mainly the self-care possibilities of the patient or the support available from his informal caregivers.

- The medication list. This list comprised all medications the patient was using at the time of discharge.

- A dietician's report. If the hospital's dietician had been involved in the care of the patient, she would write a report for the primary care team's dietician.

- A multidisciplinary report. In this section caregivers were asked to report their main findings and changes to their treatment/care plan.

Where appropriate care protocols were added to this file. Patients and informal care- 
givers also had access to the dossier. Patients themselves acted as the actual trustees of their dossier.

\section{The care protocols}

The care protocols were intended to improve the coordination of care by standardizing and supporting specific actions. These protocols were developed by a multidisciplinary team. Complete consensus on all protocols was reached.

Protocols that were introduced are:

- intravenous therapy

- epidural-spinal pain relief

- information about the Pharma/Deltec CAADD-I pain relief device being used

- information about the intravenous medication being administered by the same pump

- the pharmaceutical trajectory (See Figure 2). In this protocol the delivery arrangements of medications and sterile medical aid supplies were recorded. Because the local pharmacies had no experience in delivering some of these special medications or medical aid supplies, this protocol proved to be practical and very useful. Without it some of these products could not have been delivered at all or only in large quantities.

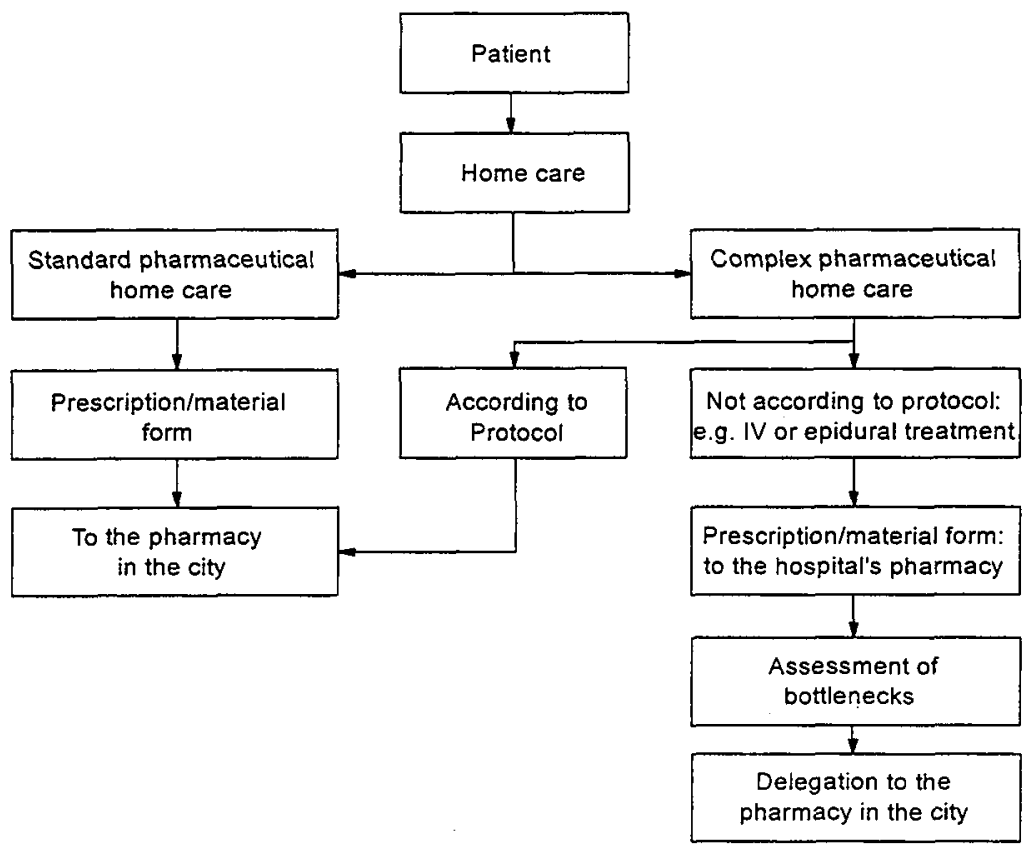

Figure 2 The pharmaceutical trajectory (IV = intravenous)

In general, all the protocols described the criteria that should be met by the patient, their caregivers and the hospital, in order to make this kind of treatment possible at home. For 
example, the patients should understand information about the equipment and its application, the general practitioner should be willing to support the patient using this procedure at home, and the hospital should provide education for primary caregivers on unfamiliar areas of medical-technology.

Furthermore, these protocols contained information about the systems used, its applications, the necessary materials for their usage, maintenance (checklist), common problems and complications and how to tackle them.

As can be seen from Figure 1, the intervention did not interfere with existing health care organizational structures. So, for patients being admitted to hospital, the medical consultant specialist retained responsibility. For those patients at home, their general practitioner was responsible.

\section{Study hypothesis (See Figure 3)}

With the introduction of this transmural home care intervention programme for terminal cancer patients, we hypothesized that it would, when compared to the standard care available in The Netherlands:

- lower re-hospitalization of these patients and enable a higher percentage of them to die at home

- contribute positively to the patient's quality of life

- contribute positively to the direct caregiver's quality of life

- lead to a better coordination of care and continuity of care

- not induce extra health care costs

By improving the coordination of care and possibly thereby the continuity of care also, patients and their direct caregivers may be better supported leading to a reduction in rehospitalization of patients and to a higher percentage of patients being able to die at home. Both are in accordance to the wishes of most patients. ${ }^{7.8}$ Furthermore, the patient's quality of life may be influenced positively, assuming that the intervention indeed leads to a better quality of care. Other factors, however, may also influence re-hospitalization and the patient's quality of life. These may be found in the following categories: the patient's social network, their disease -, and personal characteristics. ${ }^{100-102}$ These may act as confounders in this study and will have to be accounted for in the analysis.

If a lower re-hospitalization rate would be possible using this intervention, direct caregivers may feel increasingly burdened. ${ }^{103}$ However, as the direct caregiver's quality of life may be positively related to the patient's quality of life, ${ }^{103,104}$ and we assume that these direct caregivers will be better supported because of the improved continuity of care, we hypothesized that the intervention may have a positive influence on their quality of life.

Because all 4 elements of the intervention were especially designed to enhance the coordination of care, we hypothesized that the intervention would show improvements in this area. In doing so it may also have positive consequences for the continuity of care, which is regarded an essential aspect of the care process. ${ }^{49,50,105-108}$ 


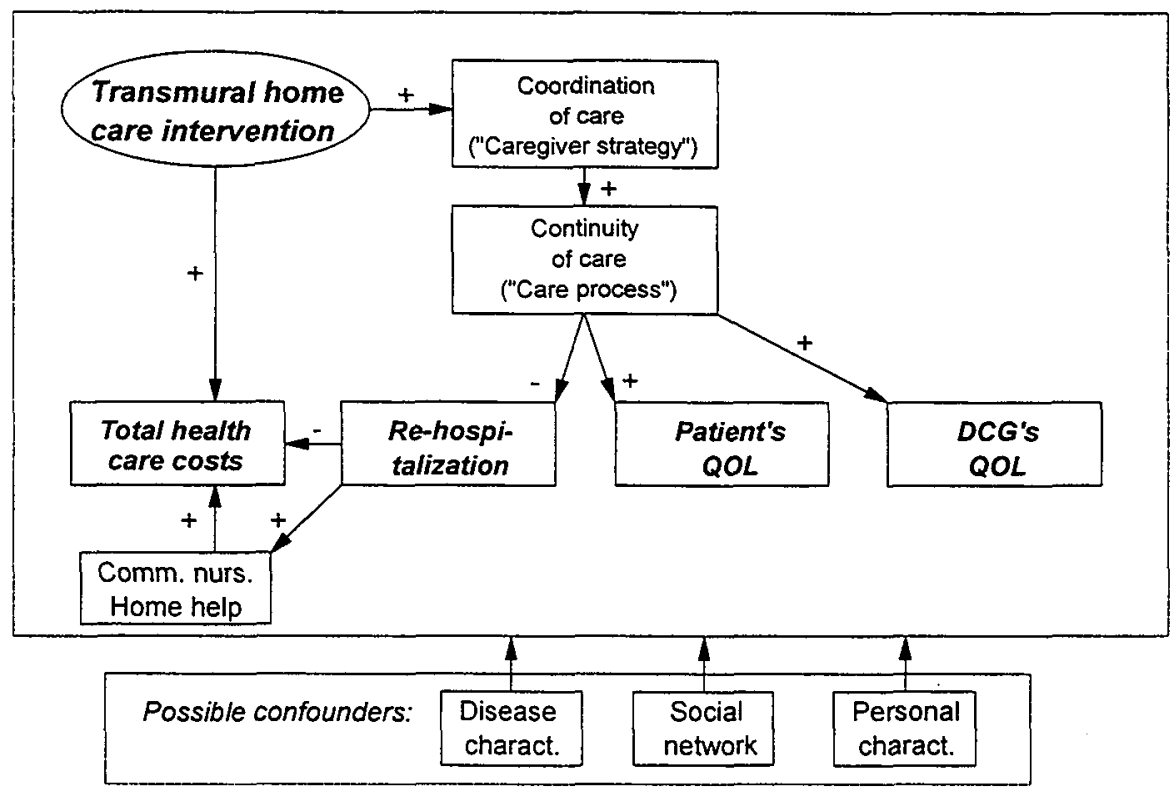

Figure 3 Study hypothesis

$D C G$ = direct caregiver; $Q O L=$ quality of life; Comm. nurs. = community nursing; charact. $=$ characteristics $;+=$ increases $/$ improves; $-=$ decreases

Although some people presume that community care would be cheaper than hospital care, we chose to hypothesize that the total health care costs would be similar for both groups. We based this hypothesis on the assumption that lower re-hospitalization rates would indeed lower hospital costs, but also, inadvertently, raise community nursing and home help costs. Therefore, we postulated that the extra costs induced by the intervention (costs for the intervention itself plus the increase in costs for community nursing and home help) would balance the costs benefits obtained by the reduction in re-hospitalization.

\section{Study design (See Figure 4)}

Due to ethical and practical reasons a randomized design was unfeasible. The main ethical reason was put forward by treating physicians who considered it unethical to submit terminally ill patients to a randomization procedure. The main practical reason was the complexity of the health care settings. A randomized design would not only have required the cooperation of the many health care organizations within the city of Eindhoven (where the hospital was located), but also that of those organizations in the surrounding urbanized areas. With a quasi-experimental design we could organize the intervention in Eindhoven initially and use the surrounding areas as a control group. Thus, patients living in Eindhoven were allocated to the intervention group and those 
from the surrounding areas to the control group. If randomization is impossible this kind of design is preferred, provided the statistical analysis is adequate. ${ }^{109-114}$

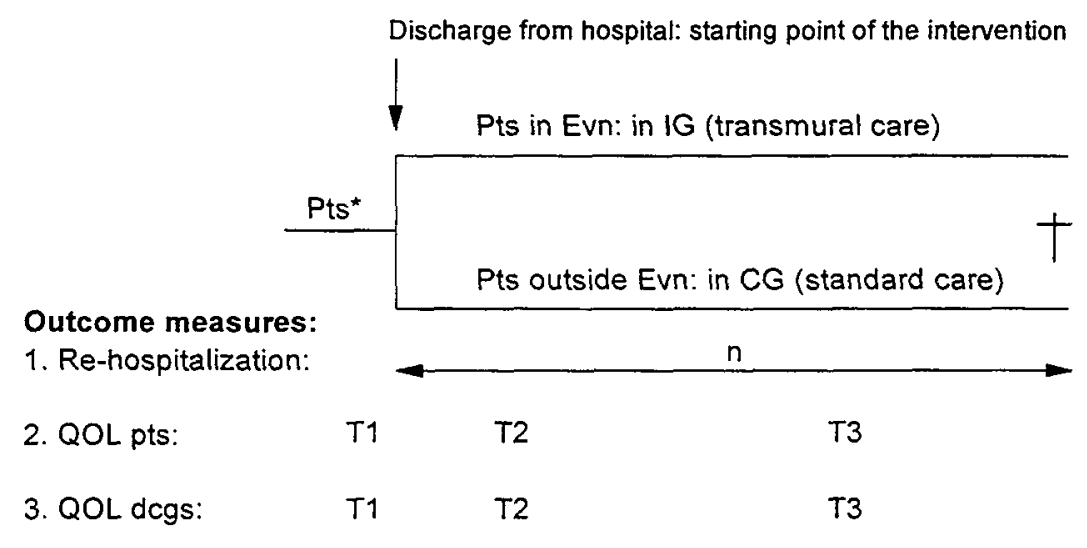

T4

Figure 4 Study design

$\mathrm{T} 1-\mathrm{T} 4$ = see text, $\mathrm{QOL}=$ quality of life, pts = patients, dcgs = direct caregivers, $\mathrm{n}=$ number of days the patient was re-hospitalized during the remainder of his life, ${ }^{*}=$ patients fulfilling the inclusion criteria, $\dagger=$ death, $I G=$ intervention group, $C G=$ control group, Evn $=$ Eindhoven .

Considering the design of the study it has to be recognized that these 2 areas are very similar. Eindhoven is a city with 200,000 inhabitants and a highly urbanized surrounding area. These urbanized surroundings also have a population of about 200,000 . The maximum distance these people have to travel to our hospital is about 30 kilometres (about 20 miles), making access to hospital easy for everyone in the study. These areas are in fact so urbanized that together they form 1 big city. Furthermore, the availability of home care services was similar in both groups. A pilot study using the data base of the Comprehensive Cancer Centre South (IKZ) did not reveal any differences in the incidence of the various cancer types, the age or gender of cancer patients in these 2 areas.

Patients who were admitted to the hospital's multidisciplinary oncology ward and who fulfilled the following inclusion criteria: incurable cancer, an estimated prognosis of less than 6 months, being fully informed concerning his disease, being older than 18 years, and willing to give an informed consent, were included in the study. Patients were asked to participate in the study by their treating consultant specialist from $1^{\text {st }}$ January 1994 till $1^{\text {st }}$ February 1995.

Upon entry to the study a broad spectrum of initial patients characteristics were collected encompassing personal -, social network -, and disease characteristics. This enabled us to search thoroughly for possible confounders in the relationships between the intervention and the various outcome measures. 
Data on re-hospitalization of patients were collected from the day the patient was initially discharged from hospital until death. Place of death was recorded.

Patient's and their direct caregiver's quality of life were measured multidimensionally 1 week before (T1), 1 week after (T2) and 4 weeks after the patients were discharged from hospital (T3). The direct caregiver's quality of life was also measured 3 months after the patient died (T4; See Figure 4). These quality of life data were gathered by 2 trained interviewers.

Cost analysis was done by retrospective data gathering using the databases of the 2 major health care insurance companies VGZ (Eindhoven) and CZ (Tilburg) and those of the various home care agencies in the hospital's region. Costs for the intervention itself, as well as costs for community nursing and home help for the patients in the intervention group, were gathered prospectively.

Within 1 week of the patient's death, all caregivers involved were approached and questioned on various aspects of coordination of care and continuity of care.

The study was approved by the hospital's Medical Ethical Committee. All included patients gave their written informed consent.

\section{Aims of the study}

The study presented in this thesis tries to find the answers to the following questions.

- What is known from the literature about the effectiveness of home care programmes for terminal cancer patients on re-hospitalization and their quality of life? This question will be addressed in Chapter 2.

- To what extent has professional care been given to patients in the intervention group, and how satisfied were the professional caregivers and patients with this kind of care? Chapter 3 will give the results of this area.

- Can the transmural home care intervention programme, as described in this chapter, reduce re-hospitalization of terminal cancer patients without this having negative effects on the patient's quality of life? Will this programme enable more patients to die at home? These results will be presented in Chapter 4.

- What are the effects of this transmural home care intervention programme on the quality of life of the patients' direct caregivers? Chapter 5 will deal with this aspect.

- Does the transmural intervention programme lead to improvements in coordination of care and continuity of care? Chapter 6 will try to answer this question.

- What are the effects of this transmural care programme on the total health care costs? These results will be presented in Chapter 7.

In Chapter 8 a synthesis of the results presented in this thesis will be given. Some methodological issues, which were not addressed in previous chapters, will be discussed. From the results of this thesis, recommendations with regard to the desired health care structure and future studies are presented.

As this thesis is compiled of articles, some reiterations will be inevitable. 


\section{References}

1. Fraumeni JF, Hoover RN, Devesa SS, Kinlen LJ. Epidemiology of cancer. In: Devita VT, Hellman S, Rosenberg SA, eds. Cancer. Principles \& practice of oncology. Philadelphia: J.B. Lippincott, 1993;196-236.

2. Steering Committee on Future Health Care Scenarios. Cancer in the Netherlands: scenarios on cancer 1985-2000. Dordrecht: Kluwer Academic Publishers, 1988.

3. Macdonald $N$. The interface between oncology and palliative medicine. In: Doyle $D$, Hanks GWC, MacDonald N, eds. Oxford textbook of palliative medicine. Oxford: Oxford University Press, 1997;11-7.

4. Morris JN, Sherwood S. Quality of life of cancer patients at different stages in the disease trajectory. J Chronic Dis 1987;40:545-56.

5. Wachtel T, Allen-Masterson S, Reuben D, Goldberg R, Mor V. The end stage cancer patient: terminal common pathway. Hosp $J$ 1988;4:43-80.

6. Lowdermilk DL. Home care of the patient with gynecologic cancer. J Obstet Gynecol Neonatal Nurs 1995;24:157-63.

7. Brown D, Roberts JA, Elkins TE, Larson D, Hopkins M. Hard choices: the gynecologic cancer patient's end-of-life preferences. Gynecol Oncol 1994;55:355-62.

8. Townsend J, Frank AO, Fermont D, Dyer S, Karron O, Wallgrove A, et al. Terminal cancer care and patients' preference for place of death: a prospective study. BMJ 1990;301:415-7.

9. van den Akker PAM, Tits MHL, Kok NM. Leven met de dood. Over terminale patienten en terminale zorg in Nederland [Living with death. Terminal patients and terminal care in The Netherlands]. Houten: Bohn Stafleu Van Loghum, 1994.

10. Field D, James N. Where and how people die. In: Clark D, ed. The future of palliative care. Buckingham: Open University Press, 1993;6-29.

11. Riley G, Lubitz J, Prihoda R, Rabey E. The use and costs of Medicare services by cause of death. Inquiry 1987;24:233-44.

12. Emanuel EJ. Cost savings at the end of life. What do the data show? JAMA 1996;275:1907-14.

13. Lubitz JD, Riley GFR. Trends in medicare payments in the last year of life. $N$ Engl $J$ Med 1993;328:1092-6.

14. Doyle D, Hanks GWC, Macdonald N. Oxford textbook of palliative medicine. Oxford: Oxford University Press, 1997. 
15. Slevin ML. Quality of life: philisophical question or clinical reality. $B M J 1992 ; 305: 466-9$.

16. Aaronson NK, Meyerowitz BE, Bard M, Bloom JR, Fawzy FI, Feldstein M, et al. Quality of life research in oncology. Past achievements and future priorities. Cancer 1991;67:839-43.

17. Cella DF. Measuring quality of life in palliative medicine. Semin Oncol 1995;22:73-81.

18. Brinkley D. Quality of life in cancer patients. $B M J 1985 ; 291: 685-6$.

19. Visser O, Coebergh JWW, Schouten LJ. Incidence of cancer in the Netherlands 1993. Utrecht: SIG Health Care Information, 1996.

20. Centraal Bureau voor de Statistiek. Statistisch jaarboek voor de statistiek [Statistical yearbook for statistics]. 's Gravenhage: Sdu/uitgeverij, 1995.

21. Coebergh JWW, van der Heijden LH, Janssen-Heijnen MLG. Cancer incidence and survival in the southeast of the Netherlands 1955-1994. Eindhoven: IKZ, 1995.

22. Farncombe ML. Ambulatory supportive care for the cancer patient. Current Opinion in Oncology 1994;6:335-9.

23. Osaba D. Effect of cancer on quality of life. London: CRC Press Inc. 1991.

24. Gersons-Wolfensberger DCM, Schade E. Patienten met kanker [Patients with cancer]. In: Schrijvers AJP, Stevens PGJJ, van Adrichem JAM, et al., eds. Handboek Thuiszorg [Manual for Home Care]. Utrecht: Elsevier/De Tijdstroom B.V. 1997;A 6.3.: 1-21.

25. Coates A, Abraham S, Kaye SB, Sowerbutts T, Frewin C, Fox RM, et al. On the receiving end - patient perception of the side effects of cancer chemotherapy. Eur J Cancer Clin Oncol 1983;19:203-8.

26. Bell DR, Tannock IF, Boyd NF. Quality of life measurements in breast cancer patients. $B r J$ Cancer 1985;51:577-80.

27. Hughes JE. Emotional reactions to the diagnosis and treatment of early breast cancer. $J$ Psychosom Res 1982;26:277-83.

28. de Haes JCJM, Raatgever JW, van der Burg MEL, Hamersma E, Neijt JP. Evaluation of the quality of life of patients with advanced ovarian cancer treated with combination chemotherapy. In: Aaronson NK, Beckmann J, eds. The quality of life of cancer patients. New York: Raven Press, 1987;215-26.

29. Reuben DB, Mor V, Hiris J. Clinical symptoms and length of survival in patients with terminal cancer. Arch Intern Med 1988;148:1586-91.

30. Ahmedzai S. Quality of life research in the European palliative care setting. In: Osaba D, ed. 
Effect of cancer on quality of life. London: CRC Press, 1991;323-32.

31. Hinds C. Suffering: a relatively unexplored phenomenon among family caregivers of non-institutionalized patients with cancer. $J$ Adv Nurs 1992;17:918-25.

32. Addington-Hall $\mathrm{J}, \mathrm{McC}$ arthy $\mathrm{M}$. Dying from cancer: results of a national population-based investigation. Palliat Med 1995;9:295-305.

33. Schmitz-Scherzer R. Sterbebegleitung--eine Last fur professionelle und familiare Helfer [Terminal care--a burden for professional and family caregivers]. Z Gerontol Geriatr 1995;28:247-51.

34. Mor V, Masterson-Allen S, Houts $P$, Siegel $K$. The changing needs of patients with cancer at home. A longitudinal view. Cancer 1992;69:829-38.

35. Jones RV, Hansford J, Fiske J. Death from cancer at home: the carers' perspective. $B M J$ 1993;306:249-51.

36. Vinokur $\mathrm{AD}$, Threath $\mathrm{BA}$, Caplan $\mathrm{RD}$, Zimmerman $\mathrm{BL}$. Physical and psychological functioning and adjustment to breast cancer. Cancer 1989;63:394-405.

37. Irvine D, Crooks D, Browne G. Psychological adjustment in women with breast cancer. Cancer 1991;67:1097-117.

38. Grobe ME, Ahmannn DL, Ilstrup DM. Needs assessment for advanced cancer patients and their families. Oncol Nurs Forum 1982;9:26-30.

39. Wool MS, Guadagnoli E, Thomas M, Mor V. Negotiating concrete needs: Short term training for high risk cancer patients. Health Social Work 1989;14:184-96.

40. Maguire GP, Lee EG, Bevington DJ, Kuchemann CS, Crabtree RJ, Comell CE. Psychiatric problems in the first year after mastectomy. BMJ 1978;1:963-5.

41. van den Borne HW, Pruyn JFA. Lotgenoten bij kankerpatienten [Fellow-sufferers amongst cancer patients]. Assen: Van Gorcum, 1985.

42. Wortman CB. Social support and the cancer patient. Cancer 1984;15 (suppl):2339-60.

43. Gordon WA, Freidenbergs I. Efficacy of psychosocial intervention with cancer patients. $J$ Consulting Clin Psychol 1980;48:743-59.

44. Schrameijer F, Brunnerberg W. Psycho-sociale zorg bij kanker. Patienten en hulpverleners over problemen en hulpaanbod [Psycho-social care in cancer. Patients and caregivers views on problems and care provision]. Utrecht: Nederlands Centrum voor Geestelijke Volksgezondheid, 1992. 
45. McWhinney IR, Bass MJ, Orr V. Factors associated with location of death (home or hospital) of patients referred to a palliative care team. Can Med Assoc J 1995;152:361-7.

46. Hunt R, Bonnet A, Roder D. Trends in the terminal care of cancer patients: South Australia, 1981-1990. Aust N Z J Med 1993;23:245-51.

47. Catalan-Fernandez JGAMAGL, Pons-Sureda O, Recober-Martinez A, Avella-Mestre A, Carbonero-Malberti JM, Benito-Oliver E, et al. Dying of cancer. The place of death and family circumstances. Med Care 1991;29:841-52.

48. Wilkes E. Dying now. Lancet 1984;i:950-2.

49. O'Hare PA, Yost LS, McCorkle R. Strategies to improve continuity of care and decrease rehospitalization of cancer patients: a review. Cancer Invest 1993;11:140-58.

50. Conkling VK. Continuity of care issues for cancer patients and families. Cancer 1989;64:290-4.

51. Herd EB. Terminal care in a semi-rural area. Br J Gen Pract 1990;40:248-51.

52. Weiss LJ. Care coordination: An integration mechanism. In: Evanshwick CJ, Weiss LJ, eds. Managing the continuum of care. Rockville, MD: Aspen Publishers, 1987;271-91.

53. de Witte LP. After the rehabilitation centre. A study into the course of functioning after discharge from rehabilitation [Thesis]. Amsterdam/Lisse: Swets \& Zeitlinger, 1991.

54. Courtens AM. Kenmerken van zorg en kwaliteit van leven bij patienten met kanker [Characteristics of care and quality of life in cancer patients; Thesis]. Maastricht: Universitaire Pers Maastricht, 1993.

55. Wood ML. Communication between cancer specialists and family doctors. Can Fam Physician 1993;39:49-57.

56. Balla JI, Jamieson WE. Improving the continuity of care between general practitioners and public hospitals. Med J Aust 1994;161:656-9.

57. McCormick IR, Boyd MA. Primary and secondary care integration. $N Z$ Med $J$ 1994;107:425-7.

58. Victor CR, Young E, Hudson M, Wallace $P$. Whose responsibility is it anyway? Hospital admission and discharge of older people in an inner-London District Health Authority. $J A d v$ Nurs 1993;18:1297-304.

59. Isaac DR, Gijsbers AJ, Wyman KT, Martyres RF, Garrow BA. The GP-hospital interface: attitudes of general practitioners to tertiary teaching hospitals. Med J Aust 1997;166:9-12. 
60. Hampson JP, Roberts RI, Morgan DA. Shared care: a review of the literature. Fam Pract 1996;13:264-79.

61. Philipsen H, Stevens FCJ. Modernization, rationality, and continuity of care: Theoretical concepts and empirical findings. Sociological Focus 1997;30:189-204.

62. Rogers $\mathrm{J}$, Curtis P. The concept and measurement of continuity in primary care. Am J Public Health 1980;70:122-6.

63. van Achterberg T. Continuity of care and client satisfaction in the community. A study of professional and non-professional care for the chronically ill [Thesis]. Maastricht: Unigraphic, 1997.

64. Austin $\mathrm{CD}, \mathrm{O}^{\prime}$ Connor $\mathrm{K}$. Case management: Components and program contexts. In: Petersen MD, White DL, eds. Care of the elderly: An information source book. Newbury Park, CA: SAGE, 1989;167-205.

65. Alter C, Hage J. Organizations working together. Newbury Park, CA: SAGE, 1993.

66. van Achterberg T, Stevens FCJ, Crebolder HFJM, de Witte LP. Coordination of care: effects on the continuity and quality of care. Int $J$ Nurs Stud 1996;33:638-50.

67. Parkes CM. Not always. J Palliat Care 1988;4:50-2.

68. Greer DS, Mor V, Morris NN, Sherwood S, Kidder D, Birnbaum H. An alternative in terminal care: results of the National Hospice Study. J Chronic Dis 1986;39:9-26.

69. Kane LK, Wales J, Bernstein L, Leibowitz A, Kaplan S. A randomised controlled trial of hospice care. Lancet $1984 ; \mathrm{i}: 890-4$.

70. Ferrell BR, Ferrell BA, Ahn C, Tran K. Pain management for elderly patients with cancer at home. Cancer 1994;74:2139-46.

71. Mercadante S. Intrathecal morphine and bupivacaine in advanced cancer pain patients implanted at home. J Pain Symptom Manage 1994;9:201-7.

72. Coyle N, Cherny $\mathbb{N}$, Portenoy RK. Subcutaneous opioid infusions at home. Oncology 1994;8:21-7.

73. Patt RB. PCA: prescribing analgesia for home management of severe pain. Geriatrics $1992 ; 47: 69-72,84$.

74. Rapp RP, Bivins BA, Littrell RA, Foster TS. Patient-controlled analgesia: a review of effectiveness of therapy and an evaluation of currently available devices. DICP $1989 ; 23: 899-904$. 
75. Trijsburg RW, van Knippenberg FCE, Rijpma SE. Effects of psychological treatment on cancer patients: a critical review. Psychosom Med 1992;54:489-517.

76. Bozzetti F. Is enteral nutrition a primary therapy in cancer patients? Gut 1994;35:S65-8.

77. King LA, Carson LF, Konstandinides N, House MS, Adcock LL, Prem KA, et al. Outcome assessment of home parenteral nutrition in patients with gynecologic malignancies: what have we learned in a decade of experience? Gynecol Oncol 1993;51:377-82.

78. August DA, Thorn D, Fisher RL, Welchek CM. Home parenteral nutrition for patients with inoperable malignant bowel obstruction. J Parenter Enteral Nutr 1991;15:323-7.

79. Zimmer JG, Groth-Junker A, McCusker J. A randomized controlled study of a home health care team. Am J Public Health 1985;75:134-41.

80. Hughes SL, Cummings J, Weaver F, Manheim L, Braun B, Conrad K. A randomized trial of the cost effectiveness of VA hospital-based home care for the terminally ill. Health Services Research 1992;26:801-17.

81. McCorkle RG, Benoliel JQ, Donaldson G, Georgiadou F, Moinpour C, Goodell B. A randomized clinical trial of home nursing care for lung cancer patients. Cancer 1989;64:1375-82.

82. Schrijvers AJP. Health and Health Care in the Netherlands. A critical Self-assessment by Dutch Experts in the Medical and health Sciences. Utrecht: De Tijdstroom B.V. 1997.

83. Stuurgroep Toekomstscenario's Gezondheidszorg. Toekomstscenario's voor eerstelijnszorg en thuiszorg [Future scenario's for primary and community care]. Houten: Bohn Stafleu Van Loghum, 1992.

84. Klaren HM, Zylicz Z. Zorgverlening aan terminale kankerpatienten. Hospice Rozenheuvel kijkt terug op de eerste honderd patienten [Provision of care for terminal cancer patients. The Rozenheuvel Hospice reviews their first hundred patients]. Medisch Contact 1996;51:253-6.

85. Polder JJ, Meerding WJ, Koopmanschap MA, Bonneux L, van der Maas PJ. Kosten van ziekten in Nederland 1994 [Costs of diseases in The Netherlands 1994]. Rotterdam: Instituut Maatschappelijke Gezondheidszorg, 1997.

86. McWhinney IR. Caring for patients with cancer. Family physicians' role. Can Fam Physician 1994;40:16-9.

87. Kruyt J, Slingerland P. Transmurale zorg in de praktijk. Tussen thuis en ziekenhuis [Transmural care in practice. Between home and hospital]. Utrecht: NIZW Uitgeverij, 1996.

88. Nationale Raad voor de Volksgezondheid/College voor Ziekenhuisvoorzieningen. Transmurale somatische zorg [Transmural somatic care]. Zoetermeer: Nationale Raad voor de Volksgezondheid, 1995. 
89. Gezondheidsraad: Commissie thuiszorg voor patienten met kanker. Thuiszorg voor patienten met kanker [Home care for cancer patients]. 's Gravenhage: Gezondheidsraad, 1991.

90. Commissie modernisering curatieve zorg. Gedeelde zorg; betere zorg [Shared care; better care]. Zoetermeer: Commissie Modernisering Curatieve Zorg, 1994.

91. Persoon A, Francke AL, Temmink D, Kerkstra A. Transmurale zorg in Nederland. Een inventarisatie op basis van bestaande gegevensbestanden [Transmural care in The Netherlands. A inventory based upon existing data files]. Utrecht: Nivel, 1996.

92. Buckling PHJ. Innovaties in samenwerking, het innovatieproject in Zuid-West Friesland [Innovations in collaboration, the innovation project of South-West Friesland]. In: Schrijvers AJP, Stevens PGJJ, van Adrichem JAM, et al., eds. Handboek thuiszorg. (Manual for Home Care). Utrecht: Elsevier/De Tijdstroom B.V. 1991;D 9.1:1-56.

93. Ministerie van WVC. Over de ontwikkeling van gezondheidsbeleid: Feiten, beschouwingen en beleidsvoornemens (Nota 2000) [The development of health care policies: Facts, observations, speculations and policy intentions (Note 2000)]. 's Gravenhage: Staatsuitgeverij, 1986.

94. Addington-Hall JM, MacDonald LD, Anderson HR, Chamberlain J, Freeling P, Bland JM, et al. Randomised controlled trial of effects of coordinating care for terminally ill cancer patients. BMJ 1992;305:1317-22.

95. Challis D, Darton R, Johnson L, Stone M, Traske K. An evaluation of an alternative to long-stay hospital care for frail elderly patients: Il costs and effectiveness. Age Ageing $1991 ; 20: 245-54$.

96. Koedoot CH, Hommel AAC, van der Horst EJ, Knipscheer CPM. De ouderen in het project individuele zorgsubsidie Rotterdam, deel II: Longitudinaal onderzoek naar de effecten van zorgbemiddeling en zorgsubsidie [The elderly in the individual care subsidy project, part II: A prospective investigation of the effects of care intercession and subsidy]. Amsterdam: Free University, 1991.

97. Ehnfors M. Nursing documentation practice on 153 hospital wards in Sweden described by nurses. Scandinavian Journal of Caring Sciences 1993;7:201-7.

98. Hooker L, Williams J. Parent-held shared care records: bridging the communication gaps. $\mathrm{Br}$ $J$ Nurs 1996;5:738-41.

99. Ellenbecker $\mathrm{CH}$, Shea $\mathrm{K}$. Documentation in home health care practice. Evidence of quality care. Nursing Clinics of North America 1994;29:495-506. 


\section{Chapter 1}

100. de Haes JCJM. Kwaliteit van leven van kanker patienten [Quality of life of cancer patients]. Amsterdam/Lisse: Swets \& Zeitlinger, 1988.

101. Evans DR, Pellizzari JR, Culbert BJ, Metzen ME. Personality, marital, and occupational factors associated with quality of life. $J$ Clin Psychol 1993;49:477-85.

102. Penninx B. Social support in elderly people with chronic diseases. Does it really help [Thesis]. Wageningen: Ponsen \& Looijen B.V. 1996.

103. McMillan SC. Quality of life of primary caregivers of hospice patients with cancer. Cancer Pract 1996;4:191-8.

104. Beckham JC, Lytle BL, Feldman ME. Caregiver burden in partners of Vietnam War veterans with posttraumatic stress disorder. $J$ Consult Clin Psychol 1996;64:1068-72.

105. Sergi-Swinehart P. Hospice home care: how to get patients home and help them stay there. Semin Oncol 1985;12:461-5.

106. Hancock BW. Palliative care of patients with terminal cancer. Current Opinion in Oncology 1993;5:655-60.

107. van der Waal MA, Casparie AF, Lako CJ. Quality of care: a comparison of preferences between medical specialists and patients with chronic diseases. Soc Sci Med 1996;42:643-9.

108. Hjortdahl P, Laerum E. Continuity of care in general practice: effect on patient satisfaction. $B M J 1992 ; 304: 1287-90$.

109. Knottnerus JA, Dinant GJ. Medicine based evidence, a prerequisite for evidence based medicine. Future research methods must find ways of accommodating clinical reality, not ignoring it. $B M J 1997 ; 315: 1109-10$.

110. McWhinney IR, Bass MJ, Donner A. Evaluation of a palliative care service: problems and pitfalls. $B M J 1994 ; 309: 1340-2$.

111. Cook TD, Campbell DT. Quasi-experimentation. Design \& analysis issues for field settings. Boston: Houghton Mifflin Company, 1979.

112. Polit DF, Hungler BP. Nursing research. Principles and methods. Philadelphia: J.B. Lippincott company, 1995.

113. Tabachnick BG, Fidell CS. Using multivariate statistics. New York: HarperCollins College Publishers, 1996.

114. Black N. Why we need observational studies to evaluate the effectiveness of health care. $B M J 1996 ; 312: 1215-8$. 


\section{CHAPTER}

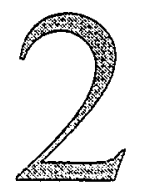

\section{The effectiveness of home care programmes for incurable cancer patients on their quality of life and re-hospitalization: A systematic review}

Presented at:

2nd International Conference Scientific Basis of Health Services \& 5th Annual Cochrane Colloquium: Amsterdam, 5-12 October 1997.

Accepted for publication as:

Smeenk FWJM, van Haastregt JCM, de Witte LP, Crebolder HFJM. The effectiveness of home care programmes for incurable cancer patients on their quality of life and rehospitalization: A systematic review. BMJ, 1997. 


\begin{abstract}
Objective - To investigate whether the general belief that comprehensive home care programmes for terminal cancer patients, compared to standard care, are effective in maintaining the patients' quality of life and reducing their hospitalization, is supported by scientific evidence.

Design - A systematic review.

Methods - A computer aided search was conducted using the databases of MEDLINE, EMBASE, Cancerlit and Psychlit. The search for, and the assessment of the methodological quality of the relevant studies were performed by two investigators, blinded from each other. Those studies investigating the effects of a home care intervention programmes on the patients' quality of life and/or their re-hospitalization in a prospective, controlled designed study, were included in the analyses.

Results - Only 9 prospective controlled studies were found. Eight were performed in the USA and one in the UK. Their methodological quality was judged moderate (median rating 62 on a 100 point scale). None of the studies showed a negative influence of home care interventions on quality of life. A significantly positive influence on the outcome measures was seen in 2 out of the 5 studies measuring satisfaction with care, in 3 out of the 7 studies measuring physical - and in 1 out of the 6 studies measuring psycho-social dimensions of quality of life, and in 2 out of the 5 studies measuring rehospitalization. The incorporation of team members able to visit patients at home and/or regular multidisciplinary team meetings into the intervention programme appeared to be related to positive results.

Conclusions - The effectiveness of comprehensive home care programmes remains unclear. Considering the enormity of problems faced by society in caring for terminal cancer patients, further research is urgently needed.
\end{abstract}




\section{Introduction}

In developed countries cancer remains one of the major causes of death, despite all the highly sophisticated treatment modalities. ${ }^{1}$ Fifty percent of all cancer patients still cannot be cured. ${ }^{2,3}$ The quality of life of these incurable cancer patients inevitably diminishes and they are frequently readmitted to hospitals. ${ }^{4-6} \mathrm{Re}$-admission is mainly due to aggravating symptoms of the disease which cannot be treated at home anymore, 'caring' has temporarily become too demanding for the informal caregivers, and/or lack of care coordination between the different professional caregivers. ${ }^{7 \cdot 10}$ The high readmission rate partly explains the high medical costs made by these patients, especially in their terminal stage, ${ }^{11}$ and is contrary to where most of these patients would like to be during this stage of their illness, i.e. at home. ${ }^{12,13}$ Therefore, home care programmes have been developed for these patients in the hope that they will be able to stay at home for longer, and maintain an acceptable quality of life. ${ }^{4,14,15}$ The main question to be answered in this paper is whether such programmes are effective or not with respect to these aims. We investigated the literature with this purpose in mind.

\section{Methods}

Publications were selected from the MEDLINE (1985-1997), EMBASE (1984-1997), Cancerlit (1979-1997) and Psychlit (1974-1997) databases. The keywords and search strategy for selecting the publications in these databases can be found in Figure 1. No language restrictions were included. ${ }^{16}$ Other search strategies were also performed, including keywords such as 'hospice', 'terminal care' and 'palliative care'. These searches, however, revealed no additional articles that could be included in our review.

["Home care" or "home nursing"] and

["Cancer" or "neoplasm(s)"] and

["Quality of life" or "well being" or "readmission" or "hospitali(s/z)ation"]

\section{Figure 1 - Search strategy and key words}

The following selection criteria were used for inclusion of studies in the analysis:

- The study population should include incurable cancer patients, and a control group against which the intervention could be compared.

- The study design should be prospective.

- The intervention must be aimed at different aspects of care with its main goal: better support for patients at home. Specific home care interventions aimed at just one aspect of care like home parenteral nutrition or pain treatment should be excluded.

- The control group should receive the standard available (home) care. Cases where the control group received only hospital care should be excluded.

- The dependent variables in the study should be one or more dimensions of quality 
of life and/or the re-hospitalization rate of patients.

Selection of the papers was performed by two investigators separately (FS and JvH) using the above mentioned inclusion criteria. A consensus meeting was planned to deal with any disagreements. If no agreement could be reached a third investigator (LdW) decided whether or not the paper should be included. The reference lists of these selected papers were screened for other relevant studies.

Criteria based analysis was performed on the selected papers in order to assess their methodological quality. Standardized analyses were performed by two investigators (FS and $\mathrm{JvH}$ ). To prevent bias, the journal name, authors list, title, summary, and discussion paragraphs were deleted from the articles and the reviewers were blinded from each other. The articles were scored using strict criteria addressing the methodological quality (See appendix and Table 1), which were based on generally accepted principles. ${ }^{17}$ Once again a consensus meeting followed to tackle any disagreements and if necessary a third investigator ( $\mathrm{LdW}$ ) was asked to give his opinion, which was then considered the final decision.

Table 1 Criteria list for methodological assessment

(OM = outcome measure; for details see appendix)

\begin{tabular}{lc}
\hline Criterion & Weight \\
\hline Study population & 6 \\
A. Selection and homogeneity & 11 \\
B. Design & 9 \\
C. Comparability of groups & 6 \\
D. Drop-out handling & 15 \\
E. Number of patients included & \\
Interventions & 15 \\
F. Interventions and standard care description & 6 \\
G. Co-interventions & \\
Effect & 5 \\
H. Blinding of the person who collected the OM & 8 \\
I. Appropriate outcome measures & 6 \\
J. Follow-up & \\
Data presentation and analysis & 10 \\
K. Statistical analysis & 3 \\
L. Data presentation & 100 \\
\hline Total &
\end{tabular}

Essential characteristics of each home care intervention programme and its main outcome measures were assessed. These included: the country in which the investigation 
took place, the base of the intervention, the kind of care the control group received and the kind of intervention that was investigated.

Data concerning re-hospitalization is presented as 're-hospitalization' for all studies so that the various results can be compared with each other. 'Re-hospitalization' was defined as the percentage of days that the patient was admitted to hospital from the start of the intervention until death. For this, we had to calculate the 're-hospitalization' in most studies using the data presented in the articles.

Finally, we tried to relate the results of the studies to their methodological quality and the type of intervention used in the studies.

\section{Results}

\section{Results of the search strategy}

The search strategy resulted in 358 articles. Of these articles 9 did meet our inclusion criteria. ${ }^{14.15,18-24}$ The overall agreement on the inclusion of articles was $100 \%$. Three articles described the results of the National Hospice study. ${ }^{19-21}$ Two of these showed considerable overlap, so they were combined. ${ }^{19,20}$ One study ${ }^{22}$ appeared to report the findings of a subgroup analysis of a previous published study. ${ }^{15}$

\section{Methodological score (See Table 2)}

The initial disagreement on the methodological score between the 2 reviewers was $9.2 \%$ (34 items in 8 articles $=272$ items that had to be scored, initially 25 items were scored differently by the reviewers). Most disagreement was caused by reading and interpretation errors, and could easily be solved in a subsequent consensus meeting by the 2 investigators ( $\mathrm{FS}$ and $\mathrm{JvH}$ ).

The scores of the methodological quality assessment ranged from 48 to 68 (mean 59 ). The median score was 62 , indicating the studies to be of moderate methodological quality. The most prevalent shortcomings were in the areas of study population homogeneity, intervention and control group comparability, the handling of drop-outs and the blinding procedure for those who collected the outcome measures.

\section{Intervention and standard care characteristics (See Table 3)}

The interventions were hospital based in 3 studies, ${ }^{14,15,22}$ hospice based in 2 studies, ${ }^{19,21}$ community based in 2 studies ${ }^{23,24}$ and based at a rehabilitation centre in 1 study. ${ }^{18}$ The intervention and standard care programmes were poorly described. One study reported having a 24-hour service for their patients. ${ }^{18}$ In 4 studies team members were able to visit patients at home. ${ }^{15,18,22,24}$ In 1 study technical home care was possible. ${ }^{23}$ In 2 studies a training programme for the patient, his or her family and friends was included. ${ }^{15,18}$ In 4 studies regular team meetings were implemented. ${ }^{14,15,18,22}$ In most studies a general practitioner and in only 1 study community nursing was available for control group patients at home. ${ }^{24}$ 


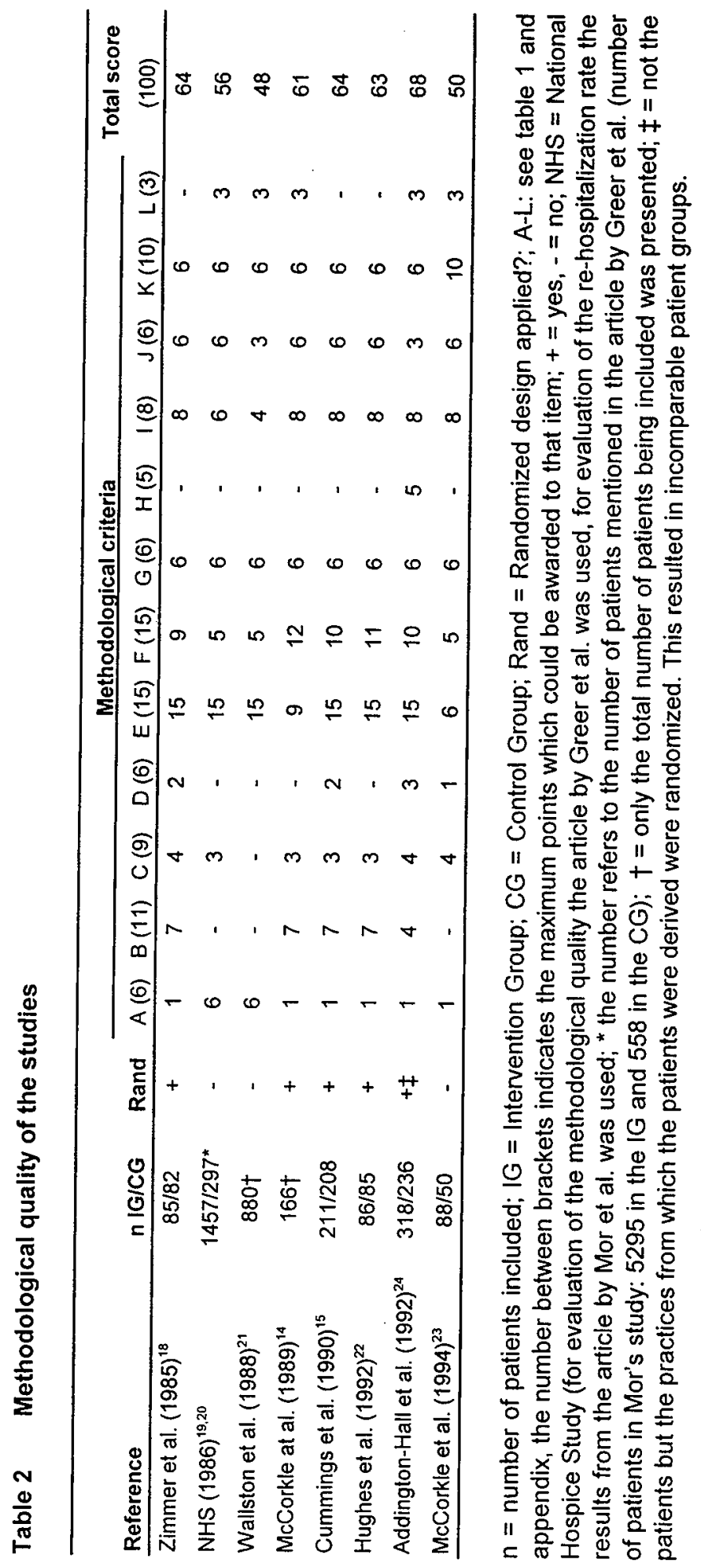




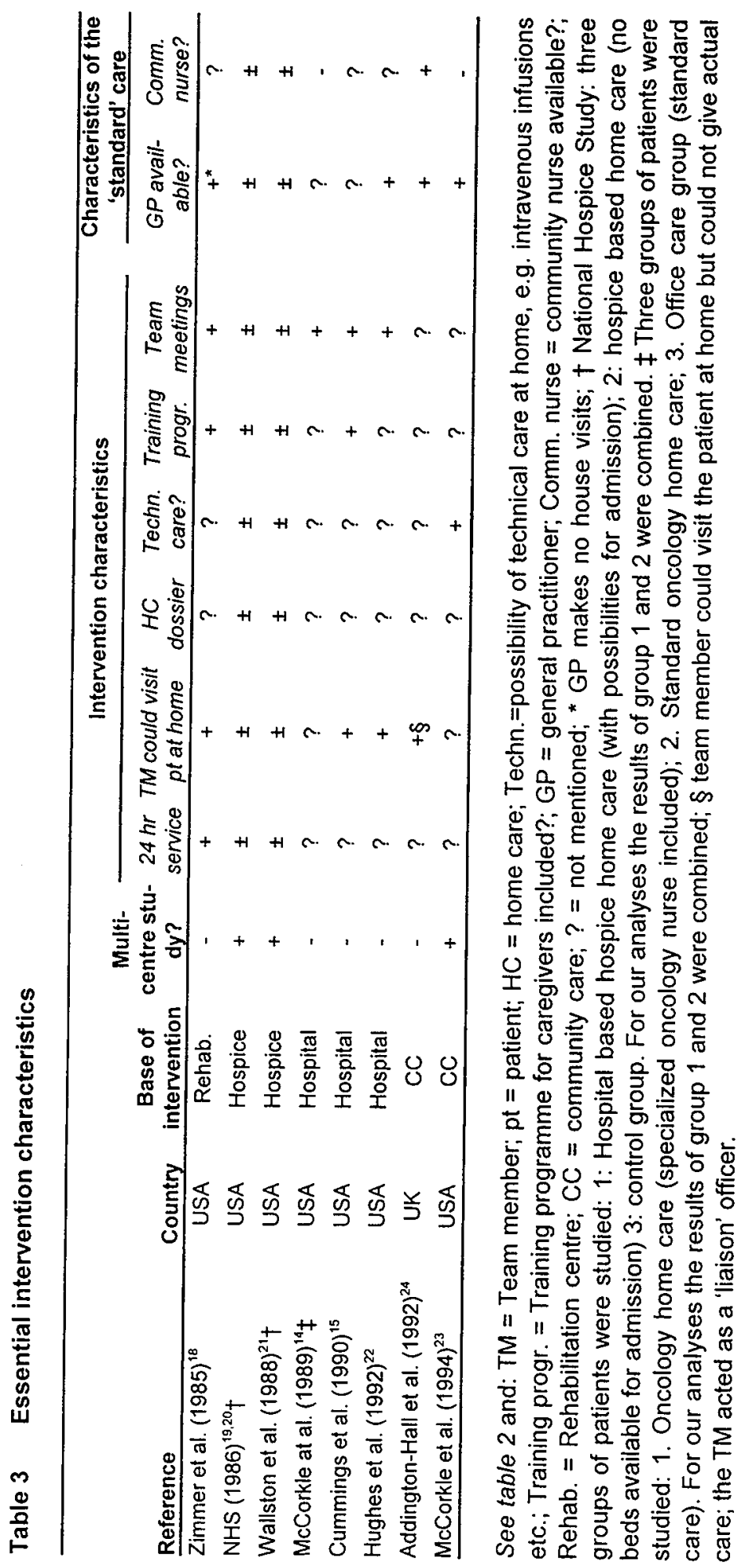




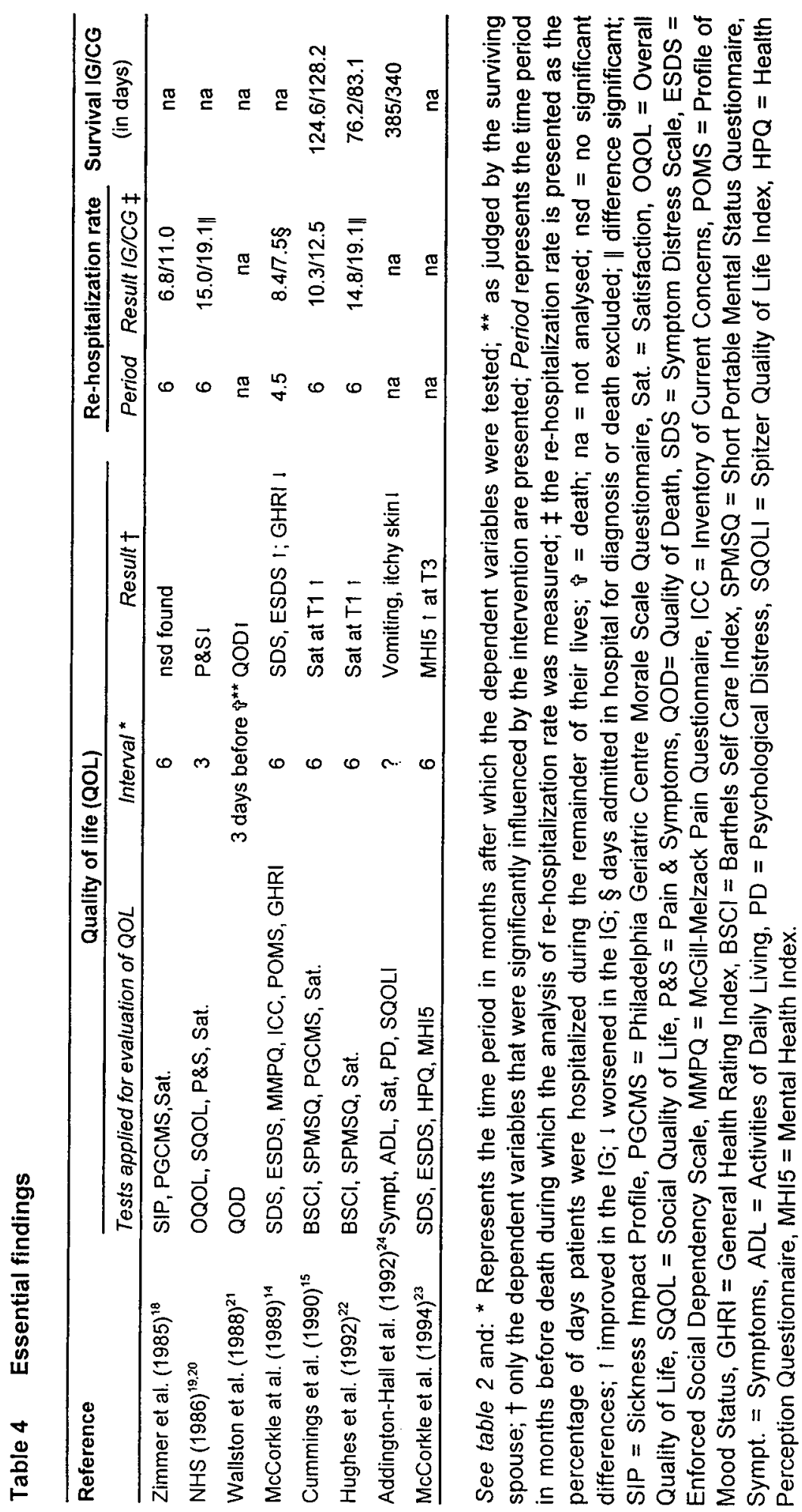




\section{Final results of the studies (See Table 4)}

Two studies ${ }^{19.24}$ reported fewer physical symptoms, and one less physical dependency of patients in their intervention group, ${ }^{14}$ whereas 4 studies did not find significant differences in this physical dimension of quality of life. ${ }^{15.18 .22 .23}$

With regard to the psychosocial dimensions, only 1 study out of the 6 investigating this topic, found a significant improvement in the 'Mental Health Index 5' questionnaire 3 months after starting the intervention. ${ }^{23}$

Of the 5 studies that investigated the satisfaction with care, 2 studies showed that patients were significantly more satisfied with the intervention programme. ${ }^{15.22}$

In 5 studies the re-hospitalization rate could be calculated. ${ }^{14,15,18,20,22}$ All but one $e^{14}$ reported a lower re-hospitalization rate for the intervention group. This difference reached statistical significance in 2 studies. $^{20.22}$

When the results of the 5 randomised studies (having the highest methodological scores) ) $^{14,15,18.22 .24}$ were compared with those of the non-randomised studies ${ }^{19-21,23}$ (having the lowest methodological scores), the outcome patterns turned out to be similar. Only 2 of the 5 randomised studies found positive effects on physical dimensions of the patient's quality of life, ${ }^{14.24}$ none found positive effects on psychosocial dimensions, two out of the 4 studies evaluating satisfaction with care showed positive results, ${ }^{15.22}$ and one out of the 4 studies that evaluated re-hospitalization found positive effects on this outcome measure. ${ }^{22}$ Whereas, only 1 of the 4 non-randomised studies (of which 2 were combined ${ }^{19.20}$ in our analyses and 1 only evaluated 'quality of death' ${ }^{21}$ ) showed positive effects on the physical dimensions, ${ }^{19}$ one on the psychosocial dimensions of the patient's quality of life, ${ }^{23}$ none of the studies showed positive effects on satisfaction with care, and one study found positive results on patient re-hospitalization. ${ }^{20}$

Finally, we tried to relate the findings of the various studies with the type of intervention being applied. This was difficult because the interventions were poorly described in most studies (Table 3). However, team members being able to visit patients at home and/or multidisciplinary team meetings being incorporated into the programme seem to be important elements for achieving favourable outcomes. ${ }^{14,15,22}$

\section{Discussion}

We found only 9 studies investigating the effects of home care programmes on the quality of life of terminal cancer patients and/or re-hospitalization. Two studies were combined because they jointly reported the results of the National Hospice Study on quality of life of patients and re-hospitalization. Furthermore, one study ${ }^{22}$ reported the results of a subgroup analysis of a previously published investigation..$^{15}$ The methodological quality of the studies was moderate considering the median score of 62 . The main shortcomings were found in the areas of population homogeneity, study design, comparability of groups, handling of drop-outs, and blinding procedures. Furthermore, the findings of the various studies failed to show a consistent pattern. None of the studies, however, found a negative influence of home care interventions on quality of life or re- 
hospitalization. Some even found a significantly positive influence on these outcome measures. No clear relation between the various findings and the methodological quality of the studies (i.e. when comparing the randomised studies with the non-randomised ones) was observed. However, team members being able to visit patients at home and/or regular multidisciplinary team meetings being incorporated into the home care programme seemed to be associated with positive findings. Because of these findings, it can be concluded that the effectiveness of home care programmes with regard to the patient's quality of life and their re-hospitalization, when compared to standard care, remains far from proven.

The results of this systematic review may, however, be criticized on several points. First, it may be questioned whether all possible relevant articles were detected. Because we, as is recommended by others, ${ }^{25-28}$

(1) searched not only the MEDLINE database but also those of Cancerlit, EMBASE, and Psychlit,

(2) made use of a combination of search terms in our literature search, using a combination of MeSH terms (indexing terms) and text words that covered a wide range of the research field,

(3) tried other search strategies too, and

(4) did not apply any language restrictions,

we are reasonably confident that we did not miss other relevant published articles that should have been included in this review.

Second, it may be argued that studies on home care intervention programmes may have been missed because we excluded those studies using hospitalized patients as their control group. These were excluded because the main goal of this review was to give an overview on the effects of these home care intervention programmes on the patient's quality of life and re-hospitalization when compared to standard (primary) care. In our literature search we only found 3 studies that compared a home care intervention programme with hospital care..$^{29-31}$

Third, the choice and rating of those items measuring the methodological quality of studies may be criticized because these are prone to subjective preferences. The choice of items, however, was based on generally accepted principles of intervention research and covers several dimension of methodological quality. ${ }^{17}$ The assignment of the various weights to the different items (Table 1), on the other hand, is by nature more subjective. If, however, others are of the opinion that other weights should be assigned to the various criteria, they can easily recalculate their 'own' methodological scores using Table 2 and their own rating list.

Fourth, it has to be recognized that 8 out of the 9 studies included in this study were from the USA. The other one was from the UK. Therefore the result of this review cannot be translated to other countries. The obvious difference between the number of studies performed in the USA compared to those performed in other parts of the world with highly developed health care systems, such as North West Europe, may be explained by its less well developed primary care system. Therefore, the need for home 
care programmes for chronically ill patients with complex problems in the USA may be higher. This difference in the primary care systems between these two parts of the world, and the fact that most of the controlled studies on the effectiveness of home care programmes were performed in the USA, shows the urgent need for further research on this subject in North West European countries.

To conclude, the effectiveness of home care programmes for terminal cancer patients still remains far from proven. This, given the extensive development of home care programmes in several countries, ${ }^{32-36}$ may be in contrast to what is generally believed by patients, professional caregivers and health care politicians. Further properly designed studies are therefore urgently needed to elucidate the effects of these home care programmes. This is particularly true for North West Europe. As we found that those programmes enabling team members to visit patients at home and/or incorporating multidisciplinary team meetings had positive outcomes, future investigators planning to research this subject are advised to incorporate these 2 elements into their programmes. 


\section{References}

1. Fraumeni JF, Hoover RN, Devesa SS, Kinlen LJ. Epidemiology of cancer. In: Devita VT, Hellman S, Rosenberg SA, eds. Cancer. Principles \& practice of oncology. Philadelphia: J.B. Lippincott, 1993;196-236.

2. Steering Committee on Future Health Care Scenarios. Cancer in the Netherlands: scenarios on cancer 1985-2000. Dordrecht: Kluwer Academic Publishers, 1988.

3. Macdonald $N$. The interface between oncology and palliative medicine. In: Doyle $D$, Hanks GWC, MacDonald N, eds. Oxford textbook of palliative medicine. Oxford: Oxford University Press, 1997;11-7.

4. Ahmedzai S. Quality of life research in the European palliative care setting. In: Osaba D, ed. Effect of cancer on quality of life. London: CRC Press, 1991;323-32.

5. van den Akker PAM, Tits MHL, Kok NM. Leven met de dood. Over terminale patienten en terminale zorg in Nederland [Living with death. Terminal patients and terminal care in The Netherlands]. Houten: Bohn Stafleu Van Loghum, 1994.

6. Field D, James N. Where and how people die. In: Clark D, ed. The future of palliative care. Buckingham: Open University Press, 1993;6-29.

7. Jones RV, Hansford J, Fiske J. Death from cancer at home: the carers' perspective. $B M J$ 1993;306:249-51.

8. Wilkes E. Dying now. Lancet 1984;i:950-2.

9. O'Hare PA, Yost LS, McCorkle R. Strategies to improve continuity of care and decrease rehospitalization of cancer patients: a review. Cancer Invest 1993;11:140-58.

10. Conkling VK. Continuity of care issues for cancer patients and families. Cancer 1989;64:290-4.

11. Lubitz JD, Riley GFR. Trends in medicare payments in the last year of life. $N$ Engl $J$ Med 1993;328:1092-6.

12. Townsend J, Frank AO, Fermont D, Dyer S, Karron O, Wallgrove A, et al. Terminal cancer care and patients' preference for place of death: a prospective study. BMJ 1990;301:415-7.

13. Dunlop RJ, Davies RJ, Hockley JM. Preferred versus actual place of death: a hospital palliative care support team experience. Palliat Med 1989;3:197-201.

14. McCorkle RG, Benoliel JQ, Donaldson G, Georgiadou F, Moinpour C, Goodell B. A randomized clinical trial of home nursing care for lung cancer patients. Cancer 1989;64:1375-82. 
15. Cummings JEB, Hughes SL, Weaver FM, Manheim LM, Conrad KJ, Nash $\mathrm{K}$, et al. Cost-effectiveness of Veterans Administration hospital-based home care. A randomized clinical trial. Arch Intern Med 1990;150:1274-80.

16. Moher $D$, Fortin $P$, Jadad AR, Juni $P$, Klassen $T$, Le Lorier J, et al. Completeness of reporting of trials published in languages other than English: implications for conduct and reporting of systemic reviews. Lancet 1996;347:363-6.

17. Moher D, Jadad AR, Nichol G, Penman M, Tugwell P, Walsh S. Assessing the quality of randomized clinical trials: an annotated bibliography of scales and checklists. Controlled Clin Trials 1995;16:62-73.

18. Zimmer JG, Groth-Junker A, McCusker J. A randomized controlled study of a home health care team. Am J Public Health 1985;75:134-41.

19. Greer DS, Mor V, Morris NN, Sherwood S, Kidder D, Birnbaum H. An alternative in terminal care: results of the National Hospice Study. J Chronic Dis 1986;39:9-26.

20. Mor V, Kidder D. Cost savings in hospice: final results of the National Hospice Study. Health Services Research 1985;20:407-22.

21. Wallston KA, Burger C, Smith RA, Baugher RJ. Comparing the quality of death for hospice and non-hospice cancer patients. Med Care 1988;26:177-82.

22. Hughes SL, Cummings J, Weaver F, Manheim L, Braun B, Conrad K. A randomized trial of the cost effectiveness of VA hospital-based home care for the terminally ill. Health Services Research 1992;26:801-17.

23. McCorkle R, Jepson C, Malone D, Lusk E, Braitman L, Buhler-Wilkerson K, et al. The impact of posthospital home care on patients with cancer. Research in Nursing and Health 1994;17:243-51.

24. Addington-Hall JM, MacDonald LD, Anderson HR, Chamberlain J, Freeling P, Bland JM, et al. Randomised controlled trial of effects of coordinating care for terminally ill cancer patients. BMJ 1992;305:1317-22.

25. Oxman $\mathrm{AD}$, Cook DJ, Guyatt GH. User's guide to the medical literature. VI. How to use an overview. JAMA 1994;272:1367-71.

26. Dickersin $\mathrm{K}$, Scherer $\mathrm{R}$, Lefebvre $\mathrm{C}$. Identifying relevant studies for systematic reviews. $B M J$ 1994;309:1286-91.

27. Haynes RB, Wilczinsky N, McKibbon KA, Walker CJ, Sinclair JC. Developing optimal search strategies for detecting clinically sound studies in MEDLNE. $J$ Am Med Informatics Assoc 1994;1:447-58. 


\section{Chapter 2}

28. Greenhalgh T. How to read a paper. The Medline database. BMJ 1997;315:180-3.

29. Ventafridda V, De Conno F, Vigano A, Ripamonti C, Gallucci M, Gamba A. Comparison of home and hospital care of advanced cancer patients. Tumori 1989;75:619-25.

30. Dessloch A, Maiworm M, Florin I, Schulze C. Krankenhauspflege versus hauskrankenpflege: Zur lebensqualitat bei terminalen tumorpatienten [Hospital care versus home care: On quality of life of patients with terminal cancer]. Psychotherapie, Psychosomatik, Medizinische Psychologie 1992;42:424-9.

31. Vinciguerra V. A comparative assessment of home versus hospital comprehensive treatment for advanced cancer patients. J Clin Oncol 1986;4:1521-8.

32. Parkes CM. Terminal care: evaluation of an advisory domiciliary service at St. Christopher's hospital. Postgrad Med J 1980;56:685-9.

33. Seale C. A comparison of hospice and conventional care. Soc Sci Med 1991;32:147-52.

34. Lang Meier $M$, Neuenschwander $H$. Hospice - a homecare service for terminally ill patients in southern Switzerland. Support Care Cancer 1995;3:389-92.

35. Tsamandouraki K, Tountas Y, Trichopoulos D. Relative survival of terminal cancer patients in home versus hospital care. Scand J Soc Med 1992;20:51-4.

36. Beck-Friis B, Strang P. The organization of hospital-based home care for terminally ill cancer patients: the Motala model. Palliat Med 1993;7:93-100. 


\section{Appendix}

\section{CRITERIA USED TO ASSESS METHODOLOGICAL QUALITY}

\section{A. Selection and homogeneity}

Inclusion and exclusion criteria clearly described: 1 point; The investigation was confined to a homogeneous study population of terminal cancer patients (prognosis $<6$ months): 5 points.

\section{B. Design}

Randomized design: 7 points; randomization procedure described and adequate: 4 points; randomization procedure inappropriate: minus 3 points.

\section{Comparability of groups}

Are the study groups comparable for: duration of the disease, age, gender, treatment, comorbidity, coping behaviour, social economic status, social network and baseline outcome measure? If so, each item scores 1 point.

\section{Drop-out handling}

Drop-out of less than 10\%: 3 points; drop-out of less than $30 \%: 2$ points; drop-out less than $50 \%$ : 1 point.

Number of drop-outs is presented for every group: 1 point. Reasons for drop-out are mentioned: 2 points.

\section{E. Number of patients included}

Smallest group after inclusion contained more than 25 patients: 6 points; more than 50 patients: 9 points; more than 75 patients: 15 points.

\section{F. Interventions and standard care description}

Participants in the intervention and/or standard care programme are described: 3 points for each description. The intervention programme and/or standard care programme is described adequately so that others can replicate it: 4 points for each description, if the programme is described partially: 2 points for each partial description. Where the intervention is based is mentioned: 1 point.

\section{G. Co-interventions}

No co-interventions: 6 points. Comparable co-interventions: 3 points.

H. Was there blinding of the person(s) who collected the outcome measures? If yes: 5 points. 
I. Were appropriate outcome measures used to test quality of life?

Clarification of the term 'quality of life' present: 2 points. Quality of life measured in a multi-dimensional way: 2 points. Explanation why this test was used: 2 points. Patients themselves judged their own quality of life: 2 points.

\section{J. Follow up}

The outcome measures were collected in the intervention and control group at equal time intervals: 3 points. The outcome measures were collected at least 1 month after starting the intervention: 3 points.

\section{K. Statistical analysis}

Author(s) investigated the possibility of drop-out due to selection: 1 point. Drop-out was not selective: 3 points. The authors corrected their findings for possible confounding factors and no confounding factors were present (as in adequately designed randomized studies): 6 points.

\section{Data presentation}

The mean of the dependent variables are presented: 3 points.

In total 34 items had to be scored. 


\section{CHAPTER}

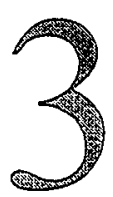

\section{Care process and satisfaction analysis of a transmural home care programme}

Accepted for publication as:

Smeenk FWJM, van Haastregt JCM, Gubbels EMAC, de Witte LP, Crebolder HFJM. Care process and satisfaction analysis of a transmural home care programme. Int $J$ Nurs Studies 1998. 


\section{Abstract}

Objective - To investigate the workload for professional caregivers (process analysis), as well as patient and caregiver satisfaction with a transmural home care intervention programme for terminal cancer patients.

Design - Prospective, descriptive.

Intervention - The intervention programme intended to optimize communication between caregivers, and enhance coordination and continuity of care. Main elements of the programme were: a specialist nurse coordinator, a 24-hour telephone service linked to a transmural home team, a home care dossier and care protocols. The intervention did not interfere with the regular health care organization.

Patients - Patients with incurable cancer and an estimated prognosis of less than 6 months were included in the study.

Methods - The workload created by the intervention for professional caregivers was prospectively recorded from inclusion until death. Patients, professional and direct (the patient's most important informal caregiver; e.g. the patient's partner) caregivers' satisfaction with the programme and its various components was investigated by means of a questionnaire.

Results - Seventy-nine patients were included in the study. Mean survival was 101.2 days. On average re-hospitalization was necessary for 5.8 days per patient during this period. Of these patients $80.8 \%$ died at home. The specialist nurse coordinator spent on average 7.5 hours on each patient case. The 24-hour telephone service was contacted 100 times in total. The transmural home team only needed to make 8 home visits to patients with problems at home. On average the patient's general practitioner visited the patient once a week. 'Intensive' community nursing care was necessary in $50 \%$ of cases. The average time spent per patient needing this kind of care was 40 minutes per day. The average daily time needed for standard community nursing was 44 minutes, and for home help 90 minutes. Patients and their various caregivers were (very) satisfied with the programme. The specialist nurse coordinator, and the 24-hour telephone service were considered essential components of the intervention.

Conclusions - Transmural home care for terminal cancer patients seems feasible given its acceptable workload for the various caregivers. As the programme did not interfere with the regular health care structures, it may easily be introduced into other hospitals and related primary care teams. The various caregivers judged the programme positively. 


\section{Introduction}

In The Netherlands, as in many other countries, health care is organized into primary care and hospital (specialist) care. Furthermore, the health care organization is characterized by its strong emphasis on community care. General practitioners have a function as 'gatekeeper' for other community or institutional services. This prevents an unbridled use of hospital care (caused by patients themselves requesting unnecessary consultations). Furthermore, primary care can include community nursing, home help, and even 'intensive' community nursing. 'Intensive' community nursing is intended for patients with a limited prognosis (less than 3 months). Where necessary, this service can provide nursing care within the home all day and night. The Dutch primary care system is highly accessible. All services are covered by compulsory national or private insurance, and require no or only limited contributions.'

Although, in theory, this health care system seems to be well organized, its imperfections are revealed when patients with complex health care problems need to be cared for at home. ${ }^{2}$ These patients are invariably cared for by many professional caregivers. Because structured communication between these caregivers forms no part of the health care structure, these caregivers often work 'parallel to each other' (unaware of the others actions) rather than 'coherently' (in clear cooperation with each other). Consequently, communication between these caregivers, as well as the coordination and continuity of care, often appears to be inadequate..$^{3-5}$ This inadequate care may have negative implications for the patient's quality of life. ${ }^{6}$

Terminal cancer patients especially, represent a group of patients in whom these care problems may arise. These patients often have many different health care problems (physical as well as psychological) ${ }^{7-9}$ and may therefore be dependent upon many different caregivers e.g. consultant specialists, their general practitioner, community nurses, home helpers and so on. ${ }^{10-13}$

To overcome these communication, coordination, and continuity of care problems, we set up a transmural home care intervention programme for terminal cancer patients. The main aim of the programme was to provide better care, tailored to meet the patient's individual needs. Whenever possible, this care was to be provided within the patient's own, safe, home environment.

In a previous study we showed that this transmural care programme resulted in a lower rate of re-hospitalization of patients, and had a positive influence on the patients' quality of life..$^{14}$ Because of this lower re-hospitalization rate, it may be assumed that the workload for professional primary caregivers will be greater or even rise to unacceptable levels.

In this study we investigated the actual workload for the various professional caregivers from the intervention and primary care teams throughout the length of the programme. Patients', professional and direct (the patient's main informal caregiver; e.g. the patient's partner) caregivers' opinions of the programme and its components were also examined. 


\section{Patients and methods}

\section{Design}

The design of this study was prospective and descriptive.

\section{The intervention}

The intervention was specifically aimed at assisting the primary care team, and consisted of four main items:

Firstly, a Specialist Nurse Coordinator was appointed. Her task was to coordinate the discharge of patients from hospital, and organize the care needed at home, tailored to meet his or her individual needs.

Secondly, a 24-hour Consultation Telephone Service was established in the Catharina hospital (the hospital from which the intervention was coordinated) in order to ensure continuity of care. A member of the primary care team could use this service if and when problems arose. The service was delivered by trained nurses from the Oncology ward and the Accident \& Emergency Department. If the problem, often a technical nursing problem, could not be solved by the caregiver in the home setting, a hospital nurse from the Transmural Home Team would pay the patient a home visit (See Figure 1). If necessary, a medical specialist was available for consultation.

Thirdly, a dossier kept at home with the patient was introduced to improve communication between the various caregivers. Each reported their findings and actions undertaken. It contained all the necessary medical and nursing information.

Fourthly, Specific Care Protocols were set up by a multidisciplinary team. These protocols helped standardize specific care and skills.

\section{Patients}

Inclusion criteria were that the patient was suffering from incurable cancer, had an estimated prognosis of less than 6 months, was aged 18 years or older, was fully informed concerning his/her diagnosis, and was already admitted onto the multidisciplinary oncological unit of the hospital. All patients were recruited between the first of January 1994 and the first of February 1995. They were followed until they died.

\section{Outcome measures}

The time spent by the specialist nurse in coordinating the care for a patient as well as the time spent in consultation with others (e.g. the community nurse, hospital nurse etc.) for performing this coordinating task, was recorded prospectively.

All calls to the 24-hour telephone consultation service in the hospital, the reasons for calling, and the person that called were recorded plus the time the nurse needed for answering and arranging the necessary actions.

The number of home visits made by the transmural home team, the reasons for these visits, as well as the time needed for these visits were recorded.

The costs for this home care intervention programme were calculated by multiplying 
the time caregivers had spent on patients with their hourly rate. In order to correct for unproductive working hours (e.g. coffee breaks etc.), this hourly rate of pay was increased by $15 \%$. Furthermore we included the amount of money needed to cover overheads costs (e.g. building, heating, furniture etc.) in this hourly rate of pay. ${ }^{15}$ Thus the hourly rates of pay for the specialist nurse coordinator, the manning of the 24-hour consultation service, and the transmural home team were calculated as being 81.48 , 64.48, 94.70 Dutch guilders (Dfl.; 1 pound sterling equaled 3.40 Dfl. in 1994) respectively.

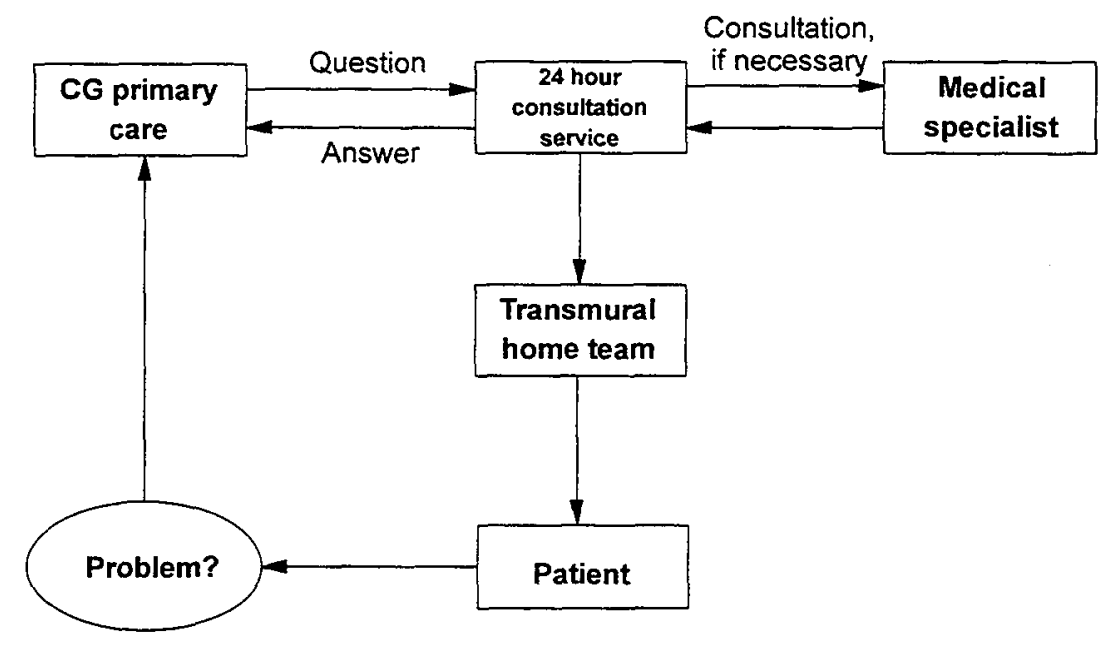

Figure 1 Organization structure of the 24-hour telephone service and the transmural home team ( $C G=$ Caregiver)

The workloads of the general practitioners, the community nurses, home helpers and volunteers were also recorded prospectively. In order to do this, records were left within the patients' homes.

After the patient had died, all (both professional and direct) caregivers involved received a questionnaire, within 1 week, asking their opinions on the various aspects of the intervention. These questions were:

1. 'In your opinion how important/necessary was the specialist nurse coordinator for the intervention?'

2. 'In your opinion how important/necessary was the 24-hour telephone service for the intervention?'

3. 'In your opinion how did the 24 -hour consultation service function?'

4. 'How troublesome was it for you to use the home care dossier?' 
5. 'Please give a mark for how the care provided to the patient actually met his/her individual needs.'

6. 'Please give a mark for the intervention as a whole.'

Questions 4 and 5 were not given to the direct caregivers. Patients were asked questions 2 and 6 one month after starting the intervention.

Questions 1-4 could be rated on a 5 point scale. A rating of 1 meant 'imperative' for questions 1 and 2, 'extremely well' for question 3, and 'extremely troublesome' for question 4. A rating of 5 indicated 'not necessary at all' for questions 1 and 2, 'extremely bad' for question 3, and 'not at all troublesome' for question 4 .

Questions 5-6 could be scored on a scale of $0-10$ where $0=$ terrible and $10=$ fantastic.

\section{Results}

\section{Patients}

The initial patient characteristics are displayed in Table 1. The mean age of the patients was 64.6 years and $59.5 \%$ of them were men. Mean survival was 101.2 days (standard deviation [SD] 142 days). The average duration of re-hospitalization during this period was 5.8 days, and $80.8 \%$ of these patients eventually died at home.

\section{The specialist nurse coordinator}

The mean time expenditures per patient of the specialist nurse coordinator performing her tasks are displayed in Table 2. Most of this time ( 7.5 hours) was spent with the patient and his/her family ( 3 hours). The mean time frame during which the coordinator was in contact with the patient was 103 days.

\section{The 24-hour telephone consultation service}

During the care process the 24-hour telephone service was contacted 100 times. In $31 \%$ of the cases the patient called, in $26 \%$ it was an informal caregiver, in $18 \%$ the patient's general practitioner and in $18 \%$ a community nurse and/or home helper. The service was contacted for medical questions (such as medication use or problems for which the consultant specialist had to be contacted) in 26 cases. Other reasons for contacting this service were nursing care problems in 25 cases, questions about the technical equipment being used at home (such as epidural pumps) in 17 cases, and psychological support in 10 cases. In 4 cases general information was asked, and in 4 cases information about the project itself. Most of the other phone calls which could not be classified under these categories were to inform the service of the patient's death.

Consultation of a medical specialist was necessary in $3 \%$ of cases, of the specialist nurse coordinator in 3\%, the transmural home team in $2 \%$, the general practitioner in $3 \%$ and the community nurse in $7 \%$ of the cases. Patients were advised to contact their general practitioner in $10 \%$, and their community nurse in $9 \%$ of cases. 
Table 1 Initial patient characteristics

\begin{tabular}{|c|c|c|}
\hline \multicolumn{3}{|c|}{ Initial patient characteristics ( $n=79)$} \\
\hline \multicolumn{3}{|c|}{ Personal characteristics } \\
\hline \multicolumn{2}{|c|}{ age $($ mean $\pm S D)$} & $64.6 \pm 10.9$ \\
\hline \multicolumn{2}{|l|}{ gender ( $\%$ male) } & $59.5 \%$ \\
\hline \multicolumn{2}{|c|}{ socio economic status: } & $44.6 \%$ \\
\hline & middle & $37.5 \%$ \\
\hline & high & $17.9 \%$ \\
\hline \multicolumn{2}{|c|}{ patients living with partner } & $67.1 \%$ \\
\hline \multicolumn{3}{|c|}{ Disease characteristics } \\
\hline \multirow[t]{4}{*}{ primary tumour: } & lung & $29.1 \%$ \\
\hline & intestine & $30.4 \%$ \\
\hline & breast & $11.4 \%$ \\
\hline & other & $29.1 \%$ \\
\hline \multicolumn{2}{|c|}{ patients with metastasis } & $73.1 \%$ \\
\hline \multirow[t]{4}{*}{ initial treatment: } & chemotherapy & $17.7 \%$ \\
\hline & operative therapy & $45.6 \%$ \\
\hline & radio-therapy & $41.8 \%$ \\
\hline & hormonal therapy & $6.3 \%$ \\
\hline \multirow[t]{4}{*}{ present treatment: } & chemotherapy & $12.7 \%$ \\
\hline & operative therapy & $2.5 \%$ \\
\hline & radio-therapy & $10.1 \%$ \\
\hline & hormonal therapy & $3.8 \%$ \\
\hline \multicolumn{2}{|c|}{ duration of the disease in months (mean $\pm S D$ ) } & $16.0 \pm 23.0$ \\
\hline \multicolumn{2}{|c|}{ patients having significant co-morbidity } & $41.0 \%$ \\
\hline
\end{tabular}

$\mathrm{n}=$ number of patients, $\mathrm{SD}=$ standard deviation.

The accessibility of the 24-hour service was excellent. Seventy-nine percent of the contacts were successful on the first attempt.

The duration of the calls was relatively short. Seventy-five percent lasted 5 minutes or less (See Table 3). The time needed for arranging any necessary actions was less than 10 minutes in $80 \%$ of cases. Only $9 \%$ of the calls were made during the night.

\section{The transmural home team}

The transmural home team only made 8 home visits to 7 different patients during the study period. One patient needed intravenous medication, 3 patients needed a new intravenous line, 2 patients needed a new line for their subcutaneous central venous catheter ('Port a Cath'), and 2 patients had problems with their urinary catheter. 
Chapter 3

Table 2 Mean time expenditures (in minutes \pm SD) per patient $(n=79)$ of the specialist nurse coordinator

\begin{tabular}{lccc}
\hline Time spent on: & $\begin{array}{c}\text { During } \\
\text { admission }\end{array}$ & $\begin{array}{c}\text { During stay at } \\
\text { home }\end{array}$ & Total \\
\hline Patient and family & $142 \pm 90$ & $40 \pm 36$ & 182 \\
Specialist & $13 \pm 15$ & $9 \pm 13$ & 22 \\
Hospital nurse & $56 \pm 35$ & $12 \pm 17$ & 68 \\
Others in hospital & $6 \pm 12$ & $2 \pm 5$ & 8 \\
General practitioner & $15 \pm 15$ & $7 \pm 15$ & 22 \\
Community nurse & $28 \pm 23$ & $15 \pm 18$ & 43 \\
Home help & $4 \pm 9$ & $3 \pm 12$ & 7 \\
Volunteer & $1 \pm 5$ & $1 \pm 4$ & 2 \\
Other informal carers & $1 \pm 4$ & $1 \pm 4$ & 2 \\
Arranging aid supplies & $3 \pm 7$ & $1 \pm 4$ & 4 \\
Reimbursement & $9 \pm 33$ & $1 \pm 2$ & 10 \\
Desk work & $57 \pm 14$ & - & 57 \\
Secr. anaesthesiology & $1 \pm 5$ & $0 \pm 1$ & 1 \\
24-hour tel. service & $1 \pm 3$ & $2 \pm 4$ & 3 \\
Rest & $18 \pm 32$ & $5 \pm 14$ & 23 \\
\hline Total & $354 \pm 198$ & $99 \pm 84$ & 453 \\
\hline
\end{tabular}

Secr. $=$ secretariat; tel. $=$ telephone

Table 3 Time needed for answering calls to the $\mathbf{2 4}$ hour telephone service itself and for arranging the necessary actions (arr. nec. actions)

\begin{tabular}{lcc}
\hline Time & $\begin{array}{c}\text { For the call itself } \\
\text { (number of calls) }\end{array}$ & $\begin{array}{c}\text { For arr. nec. actions } \\
\text { (number of calls) }\end{array}$ \\
\hline$<5$ minutes & 74 & 53 \\
$5-10$ minutes & 19 & 18 \\
$10-15$ minutes & 4 & 15 \\
$>20$ minutes & 2 & 3 \\
time unknown & 1 & 11 \\
\hline Total & 100 & 100 \\
\hline
\end{tabular}

All contacts were made during the day, except for 2 that were performed in the evening.

The mean time expenditure for these contacts was 78 minutes (range 35-120 minutes). The mean distance traveled was 13 kilometers. 


\section{Costs of the intervention programme}

The average costs per patient for the care provided by various caregivers from the intervention programme throughout the terminal phase are depicted in Table 4. This Table shows that the cost for the whole intervention averaged $641 \mathrm{Dfl}$. per patient.

Table 4 Average costs per patient (in Dfl. \pm SD) for the care given by various caregivers from the intervention programme

\begin{tabular}{lc}
\hline Caregiver & Costs \\
\hline Specialist Nurse Coordinator & $615 \pm 334$ \\
24 hour telephone service & $13 \pm 25$ \\
Transmural home team & $13 \pm 44$ \\
\hline
\end{tabular}

\section{The primary care team}

In 15 cases, time registrations by the various caregivers were inadequately recorded. So the time expenditure on patient care by the various caregivers from the primary care team could only be evaluated for 64 patients. These results are displayed in Table 5. The mean survival of these 64 patients was 75 days.

Table 5 Mean number of contacts \pm SD caregivers had with a patient and their total time expenditure for this (in hours) \pm SD during the whole care process $(N=64$ patients; mean survival 75 days)

\begin{tabular}{lcccc}
\hline Time of visit & GP $^{*}$ & CN & HH & Volunteer \\
\hline $08.00-13.00$ & $3.4 \pm 4.1$ & $38 \pm 46$ & $14 \pm 33$ & $0.8 \pm 5.5$ \\
$13.00-18.00$ & $5.8 \pm 5.8$ & $13 \pm 24$ & $4 \pm 14$ & $0.8 \pm 2.6$ \\
$18.00-23.00$ & $1.9 \pm 3.2$ & $15 \pm 27$ & $0.5 \pm 3$ & $0.4 \pm 1.7$ \\
$23.00-08.00$ & $0.6 \pm 1.4$ & $2 \pm 11$ & $7 \pm 16$ & $0.7 \pm 3.6$ \\
\hline Total time & $4.4 \pm 2.7$ & $\mathbf{5 5 . 0} \pm 115.9$ & $\mathbf{1 1 2 . 3 \pm 1 9 9 . 5}$ & $\mathbf{1 3 . 2 \pm 4 0 . 4}$ \\
\hline
\end{tabular}

GP = general practitioner, ${ }^{*}$ in 44 cases data on the time expenditure by general practitioners was recorded, $\mathrm{CN}=$ community nurse, $\mathrm{HH}=$ home help.

The mean time the community nurse spent with these patients was 44 minutes per day, and the home helper 90 minutes per day.

In 44 cases general practitioners had recorded their home visits. The mean time expenditure by the general practitioner with these patients was 4.4 hours for the whole of the care process. The mean survival of these 44 patients was 72 days.

As we were unsure whether general practitioners had recorded their visits accurately, we took a separate look at the time expenditures of a sub-group of general practitioners. This sub-group consisted of general practitioners who cared for patients also receiving 'intensive' community nursing. Because general practitioners caring for these patients 
can charge extra fees for home visits, provided they register these visits, we assumed that registration in this sub-group would be more reliable. The analysis of this sub-group revealed the same time expenditure per patient as that of the whole group of general practitioners $(n=44)$, i.e. $4.4 \pm 2.5$ versus $4.4 \pm 2.7$ hours. This equals 1 home visit of 26 minutes per week.

When comparing the time expenditure of the various caregivers from the primary care team with one another, it may be concluded that the time expenditure for community nurses is approximately 12.5 times that of the general practitioners, and the time expenditure by home helpers is twice that of community nurses. Furthermore, most general practitioners appear to visit these patients in the afternoon. This is probably due to morning consultation-hours that most have. The majority of visits by community nurses and home helpers were undertaken in the morning. This may be explained by the fact that most of these patients usually require their care in the morning (e.g. for personal hygiene, dressing etc). Overall, the care workload for volunteers was limited (mean of 11 minutes per day). However, it was relatively heavier when compared to that of the professional caregivers because $26 \%$ of their visits occurred during the night.

\section{'Intensive' community nursing}

In 66 patients, data on 'intensive community nursing' could be analysed. Of these, 33 actually received 'intensive' community nursing at some point during their care process. The mean number of hours of this nursing care was 55.6 (SD 132.5 hours) for the whole process, which is equivalent to 40 minutes per day.

\section{Opinions of patients and caregivers about the intervention programme}

These are shown in Table 6. The specialist nurse coordinator was viewed as being (extremely) important for the programme, as well as the 24-hour telephone consultation service. The telephone service was found to function between average and well. The use of the home care dossier was not too troublesome for most caregivers. The mean score given by each of the professional caregivers categories for the way care was tailored to meet the patient's actual needs ranged between 7.1 - 8.1. Patients and their various caregivers rewarded the programme with a mean score between $7.3-8.8$. Patients, their direct caregivers and the carers from the hospital care team were a little more enthusiastic than those from the primary care team.

\section{Discussion}

This study showed that the workload for the various caregivers involved in this transmural care programme seemed to be acceptable. In light of this, and the fact that the programme did not interfere with the health care organization as it currently functions in 


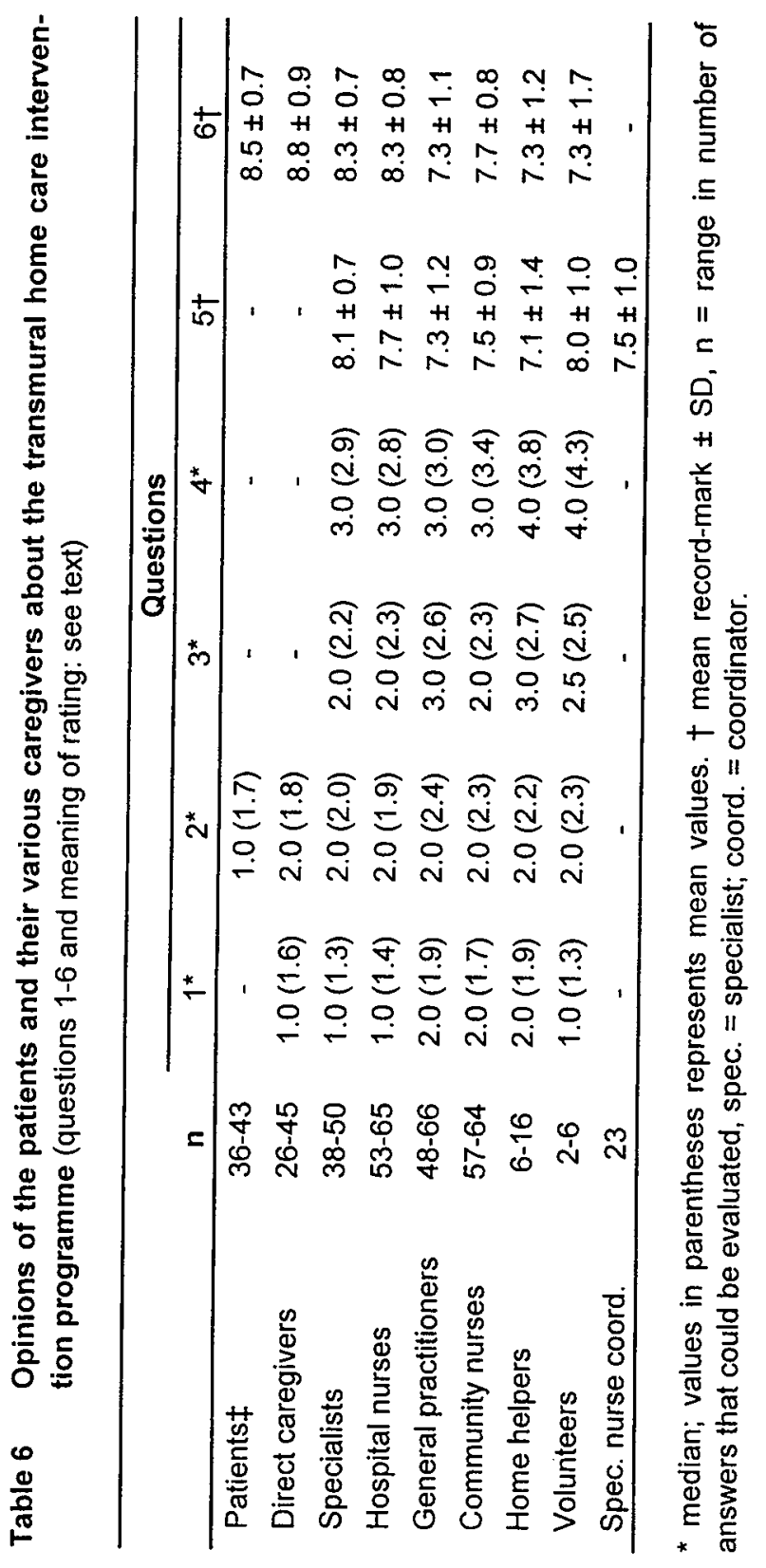


The Netherlands, it may be concluded that this transmural home care programme for terminal cancer patients is feasible and may easily be introduced by other hospitals.

Furthermore, patients' -, direct - and professional caregivers' opinions show that the specialist nurse coordinator, and the 24-hour telephone service, are seen as important components of the intervention.

Lastly, patients and their direct caregivers greatly appreciated the programme. Hospital based caregivers were a little more positive than those from primary care.

What was striking from our results was that the general practitioner appeared to have a limited role when one considers he/she visited these patients on average once a week, and assuming these visits took about 25 minutes. In contrast to this is the extensive role of community nurses and home help. Community nurses and home helpers assisted patients on average 5.1 and 10.5 hours per week respectively. Volunteers assisted for an average of 1.2 hours per week.

Most of the total time expenditure by the specialist nurse coordinator managing a case was spent in contact with the patient and his or her family. Important areas dealt with during these contacts were: explaining the transmural home team and what it can do for patients at home; analysing the home care needs of patients (besides the patient's condition, a careful assessment of the informal caregiver's caring skills is extremely important); and arranging this care accordingly.

Though the transmural home team was only requested to make 8 home visits to 7 different patients, most patients and informal caregivers were very positive about the existence of this service, and that of the 24-hour telephone consultation service. Most patients and their direct caregivers said they viewed these services as connecting structures with hospital care, like an 'umbilical cord', and therefore did not feel 'left alone'. This gave caregivers more confidence in adequately caring for patients, and patients felt easier about being cared for at home.

A literature search revealed many articles on home care programmes for terminal cancer patients. Most of these articles either described the contents of a home care programme, ${ }^{6,11,16-19}$ or the effects the programme has on patients ${ }^{20-24}$ or on health care costs. ${ }^{22.25-27}$ We only found 2 studies that also analysed the workload of a home care programme for terminal cancer patients on caregivers. ${ }^{28,29}$ Maltoni and co-workers ${ }^{28}$ evaluated the intensiveness of medical and nursing care in their home care programme for terminal cancer patients in Romagna, Italy. Comparing our results with that of Maltoni's and co-workers, our programme seems to be more intensive (a higher frequency of medical/nursing home visits: 1.4 vs 0.7 daily visits) and had more positive results (lower duration of re-hospitalization during the terminal phase of their lives: on average 5.8 vs 13.5 days and a higher percentage of patients dying at home: 80.8 vs 44.3 ). This was also true when our results were compared to those of Hinton..$^{29} \mathrm{He}$ found that terminal cancer patients cared for by the St. Christopher's Hospice home care service, had on average 3.0 visits (in our study this was 6.3 ) and 2.4 phone calls weekly from community nurses, and 0.4 visits (we recorded 1.1) weekly from general practitioners. Hinton's results on re-hospitalization were similar to ours (patients spent a median of $90 \%$ of 
their remaining days at home), but less favourable when considering the patients' place of death ( $34 \%$ of Hinton's patients died at home).

The positive opinions of caregivers and patients is in concordance with the results of other studies. ${ }^{20.21}$ Caregivers viewed the specialist nurse coordinator and the 24-hour telephone service as important parts of the intervention. This was also observed in a systematic review of the literature on this subject. ${ }^{30}$ This review found that where these items were included in a home care programme, the outcomes of re-hospitalization and/or patient's quality of life were more likely to be better.

In conclusion, our transmural home care programme did not appear to overburden professional primary caregivers, and was viewed positively (score 7.3-8.8) by patients and all their caregivers. In light of these results and because the programme, when compared to standard care, significantly lowered patient rehospitalization and had a positive effect on both their and their direct caregivers' quality of life, ${ }^{14,31}$ and did not incur extra health care costs, ${ }^{15}$ the further development of such home care programmes seems a logical recommendation. For those planning to set up such programmes the installation of a specialist nurse coordinator and 24-hour telephone service is advisable because these programme components were generally viewed as essential by patients and their various caregivers. Future research may best be directed towards examining the effects of an intervention has on the 'burden of caring' experienced by informal caregivers, and/or costs from a societal perspective. 


\section{References}

1. Schrijvers AJP. Health and Health Care in the Netherlands. A critical Self-assessment by Duth Experts in the Medical and health Sciences. Utrecht: De Tijdstroom B.V. 1997.

2. Philipsen H, Stevens FCJ. Modernization, rationality, and continuity of care: Theoretical concepts and empirical findings. Sociological Focus 1997;30:189-204.

3. Haines A, Booroff A. Terminal care at home: perspective from general practice. $\mathrm{Br} M e d J$ (Clin Res Ed) 1986;292:1051-3.

4. de Witte LP. After the rehabilitation centre. A study into the course of functioning after discharge from rehabilitation [Thesis]. Amsterdam/Lisse: Swets \& Zeitlinger, 1991.

5. van Achterberg T. Continuity of care and client satisfaction in the community. A study of professional and non-professional care for the chronically ill [Thesis]. Maastricht: Unigraphic, 1997.

6. Coyle N. Supportive care program, pain service, Memorial Sloan-Kettering Cancer Center. Support Care Cancer 1995;3:161-3.

7. Addington-Hall $\mathrm{J}, \mathrm{McC}$ arthy $\mathrm{M}$. Dying from cancer: results of a national population-based investigation. Palliat Med 1995;9:295-305.

8. Vinokur AD, Threath BA, Caplan RD, Zimmerman BL. Physical and psychological functioning and adjustment to breast cancer. Cancer 1989;63:394-405.

9. Irvine D, Crooks D, Browne G. Psychological adjustment in women with breast cancer. Cancer 1991;67:1097-117.

- 10. Mor V, Masterson-Allen $S$, Houts $P$, Siegel $K$. The changing needs of patients with cancer at home. A longitudinal view. Cancer 1992;69:829-38.

- 11. Given BA, Given CW. Family home care for individuals with cancer. Oncology (Huntingt) 1994;8:77-83.

12. Grobe ME, Ahmannn DL, Ilstrup DM. Needs assessment for advanced cancer patients and their families. Oncol Nurs Forum 1982;9:26-30.

13. Wool MS, Guadagnoli E, Thomas M, Mor V. Negotiating concrete needs: Short term training for high risk cancer patients. Health Social Work 1989;14:184-96.

14. Smeenk FWJM, de Witte LP, van Haastregt JCM, Schipper RM, Biezemans JPH, Crebolder HFJM. Transmural care. A new approach in the care for terminal cancer patients: Its effects on re-hospitalization and quality of life. Patient Educ Couns 1997; accepted. 
15. Smeenk FWJM, Ament AJHA, van Haastregt JCM, de Witte LP, Crebolder HFJM. A cost analysis of transmural home care for terminal cancer patients. Patient Educ Couns 1997; accepted.

16. Beck-Friis B, Strang P. The organization of hospital-based home care for terminally ill cancer patients: the Motala model. Palliat Med 1993;7:93-100.

17. Pannuti F. The Bologna Eubiosia Project: hospital-at-home care for advanced cancer patients. J Palliat Care 1992;8:11-7.

18. Handy CM. Patient-centered high-technology home care. Holist Nurs Pract 1989;3:46-53.

19. Ophof J. Experiences in home care of cancer patients. A new approach in cancer nursing. Acta Oncol 1989;28:35-8.

20. Beck-Friis B, Strang P, Sjoden PO. Caring for severely ill cancer patients. A comparison of working conditions in hospital-based home care and in hospital. Support Care Cancer 1993;1:145-51.

21. Hughes SL, Cummings J, Weaver F, Manheim L, Braun B, Conrad K. A randomized trial of the cost effectiveness of VA hospital-based home care for the terminally ill. Health Services Research 1992;26:801-17.

22. Ventafridda V, De Conno F, Vigano A, Ripamonti C, Gallucci M, Gamba A. Comparison of home and hospital care of advanced cancer patients. Tumori 1989;75:619-25.

23. McCorkle RG, Benoliel JQ, Donaldson G, Georgiadou F, Moinpour C, Goodell B. A randomized clinical trial of home nursing care for lung cancer patients. Cancer 1989;64:1375-82.

24. Vinciguerra V. A comparative assessment of home versus hospital comprehensive treatment for advanced cancer patients. $J$ Clin Oncol 1986;4:1521-8.

25. Cummings JEB, Hughes SL, Weaver FM, Manheim LM, Conrad KJ, Nash K, et al. Cost-effectiveness of Veterans Administration hospital-based home care. A randomized clinical trial. Arch Intern Med 1990;150:1274-80.

26. McCusker J, Stoddard AM. Effects of an expanding home care program for the terminally ill. Med Care 1987;25:373-85.

27. Vinciguerra V. Comparative cost analysis of home and hospital treatment. Prog Clin Biol Res 1986;216P: 155-64.

28. Maltoni M, Derni S, Innocenti MP, Rinaldi A, Amadori D. Description of a home care service for cancer patients through quantitative indexes of evaluation. Tumori 1991;77:453-9. 


\section{Chapter 3}

29. Hinton J. Services given and help perceived during home care for terminal cancer. Palliat Med 1996;10:125-34.

30. Smeenk FWJM, van Haastregt JCM, de Witte LP, Crebolder HFJM. The effectiveness of home care programmes for incurable cancer patients on their quality of life and re-hospitalization. A systematic review. BMJ 1997; accepted.

31. Smeenk FWJM, de Witte LP, van Haastregt JCM, Schipper RM, Biezemans JPH, Crebolder HFJM. Transmural care of terminal cancer patients: Effects on the quality of life of direct caregivers. Nurs Res 1997; accepted. 


\section{CHAPTER}

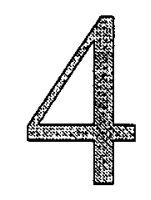

\section{Transmural care. A new approach in the care for terminal cancer patients: Its effects on re-hospitalization and quality of life}

Accepted for publication as:

Smeenk FWJM, de Witte LP, van Haastregt JCM, Schipper RM, Biezemans HPH, Crebolder HFJM. Transmural care. A new approach in the care for terminal cancer patients: Its effects on re-hospitalization and quality of life. Patient Educ Couns 1997. 


\begin{abstract}
Despite their wishes, terminal cancer patients are frequently readmitted to hospitals. This appears in part to be due to poor communication amongst professional caregivers and/or the overburdening of their (informal) caregivers. This quasi-experimental study investigated the effects of a transmural home care programme on re-hospitalization, quality of life and place of death for terminal cancer patients. The programme intended to optimize communication, cooperation and coordination between intra- and extramural health care organizations (transmural care). Initial patient characteristics of the intervention group $(n=79)$ matched those of the control group $(n=37)$ well. When compared to the control group, which received the standard community care, patients in the intervention group underwent significantly less re-hospitalization during the terminal phase of their illness $(5.8$ versus 11.5 days; $p<.05$ ) while the intervention contributed significantly positive to the patients' 'physical' quality of life 1 month after the start of the intervention. A higher, but not significant $(p=.06)$ percentage of patients in the intervention group also died at home ( 81 versus $65 \%$ ). The introduction of measures to enhance coordination and cooperation of intra- and extramural care, seems to be an improvement compared to standard community care.
\end{abstract}




\section{Introduction}

Cancer is one of the major causes of death in developed countries. ${ }^{1}$ It can have detrimental effects on quality of life, especially if the disease is incurable, ${ }^{2}$ as is true for $50 \%$ of cases. ${ }^{3.4}$ If the disease progresses and the patient enters a (pre-) terminal phase, hospital admission will be necessary in most cases. ${ }^{5.6}$ The most frequently observed reasons for admission are: inadequate alleviation of symptoms at home, physical and/or psychological inability of the direct caregiver to care for the patient and inadequate communication amongst the professional caregivers. ${ }^{7-12}$

In Britain and the United States of America, hospices were founded in the 1970s and 1980 s to provide a better form of palliative care for terminal patients. ${ }^{2}$ Others have tried to improve care by setting up 'home care programmes' based from hospices or hospitals. ${ }^{13-16}$ However, studies investigating the effects of these home care programmes, developed and carried out by these intramural organizations, failed to show a consistent positive effect on the patients' quality of life or re-hospitalization. ${ }^{13-16}$

In The Netherlands terminal cancer patients are also frequently re-hospitalized, despite the presence of a primary care team. ${ }^{17}$ The primary care team consists of a general practitioner (this service is available for consultation 24 hours a day), a community nurse (also available for 24 hours a day if necessary), a Home Help service, and a medical aid supply service which can provide special equipment for use at home for the patient e.g. special beds, equipment for epidural analgesia et cetera. Furthermore, most cancer patients are regularly checked by their hospital consultant specialist. So care in The Netherlands, as in most other developed countries, is organized into 'intra-' (hospital based) and 'extra-mural' (community based) care. As professional carers in these different sections usually confine their work to their own units, good cooperation and communication between them is often lacking. This may lead to inadequate care and unnecessary hospital admissions. ${ }^{9.10,18,19}$

In order to be able to provide better supportive care for these patients at home, an intervention programme was developed. Its main objective was to offer the patient care specially tailored to meet his individual needs, and provided by professional caregivers from primary and hospital teams. In the intervention group the primary care team continued to care for the patient as usual, but with collaborative support from the hospital care team. The intervention intended to optimize care by improving the continuity of care and by emphasizing the complementary approach to care giving, with maximal coordination and communication. This kind of care was named transmural care in The Netherlands. It has been defined by the National Council for Public Health Care as follows: ${ }^{20}$ 'Transmural care encompasses those kinds of care which are tailored to meet patient's needs. It is provided by caregivers from primary and hospital teams on the basis of coordination and cooperation, with shared responsibility and specification of delegated responsibilities.' The development of such care at a regional level was highly recommended by the Dutch government. ${ }^{21}$

It was thus hypothesized that by improving communication, coordination and 
continuity of care this transmural care would lower re-hospitalization. A desire also shared by patients. ${ }^{22-24}$ However, this should have no negative consequences for the patient's quality of life. Furthermore it should allow more patients to die at home.

\section{Patients and methods}

\section{Design of the study (Figure 1)}

Due to ethical and practical reasons the study was quasi-experimental..$^{25.27}$ Physicians who considered it unethical to submit terminally ill patients to a randomization procedure formed the main ethical reason for not choosing an experimental design. The main practical reason was due to the complexity of the health care settings. A randomized design would not only have required the cooperation of the many health care organizations within the city of Eindhoven (where the hospital was located), but also that of those organizations in the surrounding urbanized areas. With a quasi-experimental design the intervention could be organized in Eindhoven initially and the surrounding areas could be used as a control group. Thus, cancer patients being admitted in hospital, and who were living in Eindhoven were allocated to the intervention group and those from the surrounding areas to the control group.

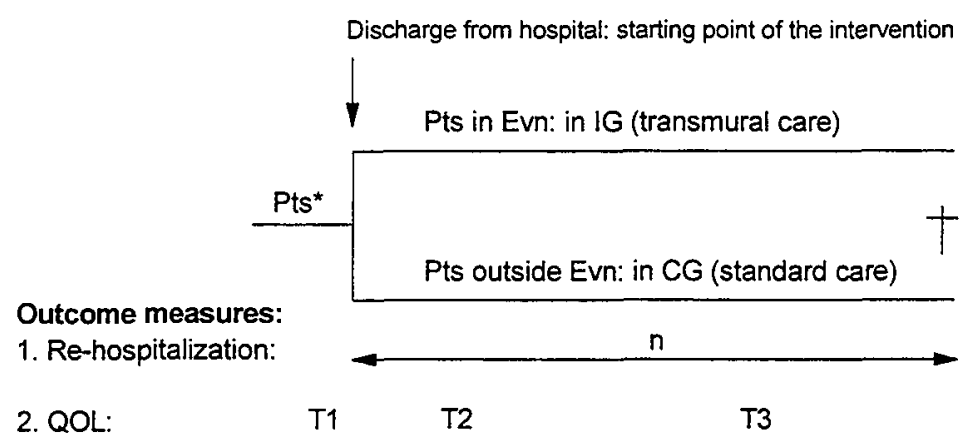

\section{Figure 1 Study design}

$\mathrm{Pts}^{*}=$ eligible patients, Evn $=$ Eindhoven, $\mathrm{QOL}=$ quality of life, $1 \mathrm{G}=$ intervention group, $C G=$ control group, $n=$ number of days re-hospitalized, $T 1$ T3: see text, $\dagger=$ death)

After discharge from hospital (i.e. the start of the intervention), patients in both groups received the standard community care available in The Netherlands. ${ }^{17}$ Next to this care the patients in the intervention group were also offered the intervention programme.

Re-hospitalization and quality of life were considered primary outcome measures. Data on the re-hospitalization of patients were collected from the day of discharge (i.e. the start of the intervention) until death. Patient's quality of life was measured 1 week 
before (T1; pre-intervention measurement), 1 week after (T2) and 4 weeks after the patients were discharged from hospital.

When considering the design of the study it should be recognized that these 2 study areas are very similar. Eindhoven is a city with 200,000 inhabitants and a highly urbanized surrounding area. These areas are in fact so much urbanized that together they form 1 big city. The Dutch government has therefore recently decided to merge Eindhoven and its surrounding municipalities into one city province. Furthermore, the availability of home care services was similar in both groups and a pilot study using the data base of the Comprehensive Cancer Centre South did not reveal any significant differences in the incidence of the various types of cancer, mean age, and gender of the cancer patients in these 2 populations.

The study was approved by the hospital's Medical Ethical Committee of the hospital. All patients gave their written informed consent.

\section{The intervention programme}

The transmural home care intervention programme was specifically aimed at assisting the primary care team, and consisted of four main items: a specialist nurse coordinator, a 24-hour telephone service in the hospital with access to a transmural home team, a collaborative home care dossier (case file), and protocols designed for specific care.

\section{The specialist nurse coordinator}

The specialist nurse coordinator is the key person in this intervention programme. On request of the treating specialist, she prepares the necessary patient discharge arrangements. Patient's wishes and (care) needs are assessed by her as well as the possibility of patient support by informal caregivers. Taking these findings into account she plans and arranges home support by professional caregivers, which will naturally be tailored to meet the patient's individual needs. In order to carry this out successfully, she needs to have daily contact with a wide variety of caregivers, from medical specialists to home helpers. She monitors the care provision process, tracks down and solves possible defaults or shortcomings.

The discharge of patients in the control group was planned as usual by the hospital nurse responsible for his/her care.

\section{The 24-hour telephone service and transmural home team}

A 24-hour telephone consultation service was installed on the hospital's multidisciplinary oncology ward (Figure 2). This service is manned by nurses from the ward. In order to do so, they were specially trained in giving assistance to patients by telephone. In case of problems arising at home this service can be contacted for advice. It can be reached day or night by telephone via a direct line (no operator delays or interference). If medical questions arise, a specialist consultant can be contacted using the service. In order to give adequate advice, a copy of the patient's home care dossier, containing all the essential medical and nursing information, is at hand. Thus, continuity 
of the care is guaranteed.

If specific nursing problems can not be solved by the primary care team, support is provided by trained nurses from the hospital's transmural home team on request by the patient's general practitioner. In general, problems which the team are asked to solve involve the high technology devices sometimes used in treatment. The home team consists of nurses from the hospital's casualty and 'day care' departments. During on call hours they can be contacted by semaphone.

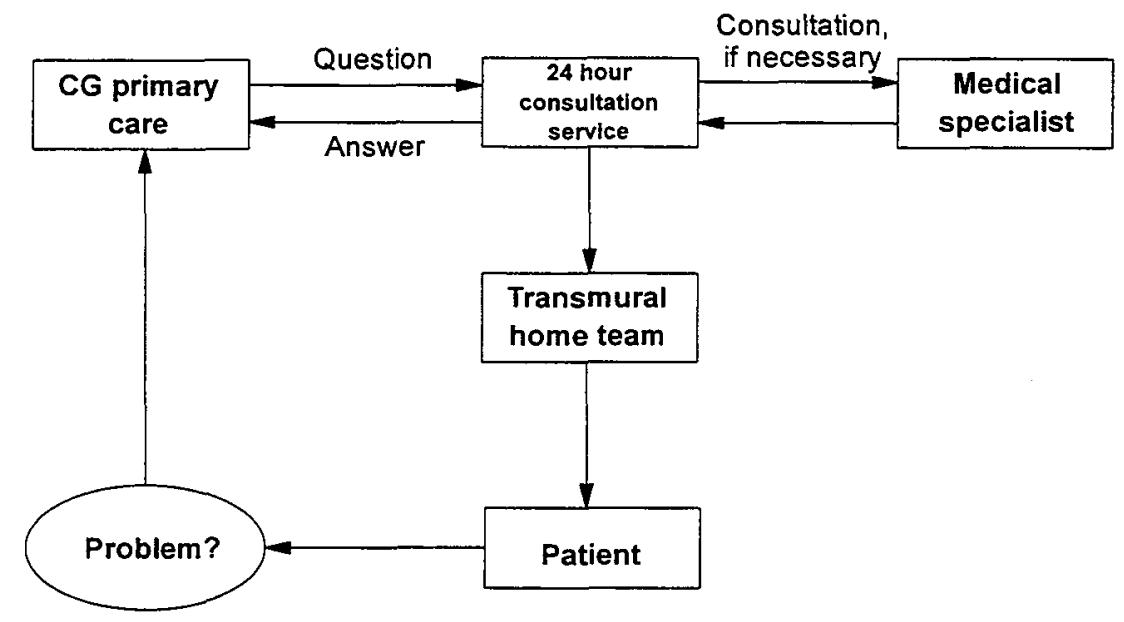

Figure 2 Organization structure of the 24-hour consultation service and the Transmural Home Team ( $C G=$ caregiver)

The home care dossier

The home care dossier was developed to improve communication between caregivers and enhance the coordination of care. Caregivers from primary - and hospital care teams were asked to collaborate in reporting their findings and actions undertaken. All caregivers are hereby informed of findings and care plans. The dossier consists of the following items: an informed consent, a list of those caregivers involved in the care of this patient, a preliminary discharge report for the general practitioner, a nursing transfer report for the community nurse, a transfer report for home helpers dealing mainly with the patient's self-care capacity or the support available from his informal caregivers, the medication list, a dietician's report, and a multidisciplinary report. In this last section caregivers are asked to report their main findings and changes to their treatment/care plan. Where appropriate, care protocols are added to this file. Patients and informal caregivers also have access to the dossier. Patients themselves act as the actual trustees of their dossier.

\section{The care protocols}

The care protocols are intended to improve the quality of the care provided at home. 
These protocols were developed by a multidisciplinary team. The protocols standardize and support caregivers when using specific skills and actions. Protocols that were introduced are: intravenous therapy, epidural-spinal pain relief, and the pharmaceutical trajectory. In the protocol for the pharmaceutical trajectory the delivery arrangements of medications and sterile medical aid supplies are laid down.

As can be seen from Figure 2, the intervention does not interfere with existing health care organizational structures. So, the responsibility for patients being admitted to hospital remains with the medical consultant specialist, and for patients at home with the general practitioner.

\section{Patients}

Those patients who were admitted to the multidisciplinary oncology ward of the hospital and who met the following inclusion criteria: cancer, an estimated prognosis of less than 6 months, age 18 years or older and being fully informed of diagnosis could be included in the study. Patients were included from the first of January 1994 till the first of February 1995.

In order to be able to correct for possible confounders in this study, we collected a broad spectrum of initial patient characteristics upon entry to the study. These included data concerning the patients' disease and its treatment, their social network and social support, ${ }^{28}$ their socio-demographic and personal characteristics (coping as measured by the short version of the Utrecht Coping List, ${ }^{29}$ premorbid health status as measured by the General Health Perception Questionnaire, ${ }^{30}$ and religion).

\section{Outcome measures}

Data on re-hospitalization was recorded as the total number of days that the patient spent in hospital during the remainder of his life.

The patient's quality of life was determined in a multi-dimensional way, using seven different instruments comprising the following dimensions: pain (visual analogue score), ${ }^{31}$ somatic and psychological complaints (Rotterdam Symptom Checklist), ${ }^{32}$ daily functioning (Sickness Impact Profile-68), ${ }^{33,34}$ fear (Spielberger State-Trait Anxiety Inventory), ${ }^{35}$ loneliness ${ }^{36-38}$ and general well-being. ${ }^{39,40}$ All information was obtained by two trained interviewers using structured questionnaires.

To yield an aggregate score for the quality of life measurements, principal component analyses with orthogonal rotation (method varimax) were performed at each assessment stage ${ }^{27}$ Identical factors were extracted at each analysis. These being:

1. a factor representing 'psycho-social functioning' (with high factor loadings for general well-being, fear, loneliness and psychological complaints in each analysis at $\mathrm{T} 1 / \mathrm{T} 2 / \mathrm{T} 3$ );

2. a factor representing 'physical functioning' (with high factor loadings for somatic complaints, pain and Sickness Impact Profile in each analysis at T1/T2/T3).

The sum of the standardized variables (' $z$-scores') that loaded high on each separate factor were used as primary outcome measures. In all standardization procedures the 
mean scores and standard deviations of the 7 individual quality of life outcome measures obtained at $\mathrm{T} 1$ were used.

Whether the patient died at home or in hospital, and the survival time after joining the study were considered as secondary outcome measures.

\section{Analyses}

Appropriate tests were used for comparing the variables according to their distribution. ${ }^{41}$ A p-value of .05 or less was considered significant, except for the analyses performed for detecting possible confounders. Here, a conservative significance level of $p \leq .10$ was used.

Only the patient data on quality of life that could be obtained at T1, T2 and/or T3, were used in the analyses. The other patients were considered 'drop-outs' for this outcome variable. To check for selection bias the initial patient characteristics of the final study group and the 'drop-outs' were compared.

Before assessing whether the intervention contributed positively to the outcome measures, a search for possible confounders in the relationship between the experimental condition and the various outcome measures was performed. For this, the correlations between the initial patient characteristics and the outcome measures were assessed. Initial patient characteristics which were significantly related to the outcome measures and which also showed a significant difference between the intervention and control group at $\mathrm{T} 1$, were considered potential confounders.

To assess whether the intervention contributed positively to re-hospitalization, 'psycho-social' or 'physical functioning', hierarchical backward multiple regression analyses were performed on these outcome measures. ${ }^{27}$ To control for initial differences in the quality of life outcome measures and the potential confounders, the pre-intervention value (value at T1) of 'psycho-social' and 'physical functioning' as well as the potentially confounding patient characteristics factors were added to the experimental condition as independent variables in the regression analyses on the quality of life outcome measures.

\section{Results}

\section{Patients}

Seventy-nine patients were included in the intervention and 37 in the control group. With regards to the re-hospitalization analysis, all patients could be studied until death.

Fifty-four patients were unable to perform the pre-intervention (T1) quality of life assessment. In most cases this was due to them feeling too ill. Of the remaining 62 patients, $17(27.4 \%)$ 'dropped-out' for the quality of life analyses because they were unable to perform one or more post-intervention measurements. This was mainly due to death or the patient feeling too 'burdened'. Figure 3 gives the reasons in more detail. Overall drop-out in the intervention group was not significantly different from that in the 
control group. Comparison of the initial patient characteristics of the 'drop-outs' with those of the patients who could be evaluated, showed no significant differences.

The essential initial patient characteristics are listed in Table 1. The 2 groups appeared to be fairly comparable.

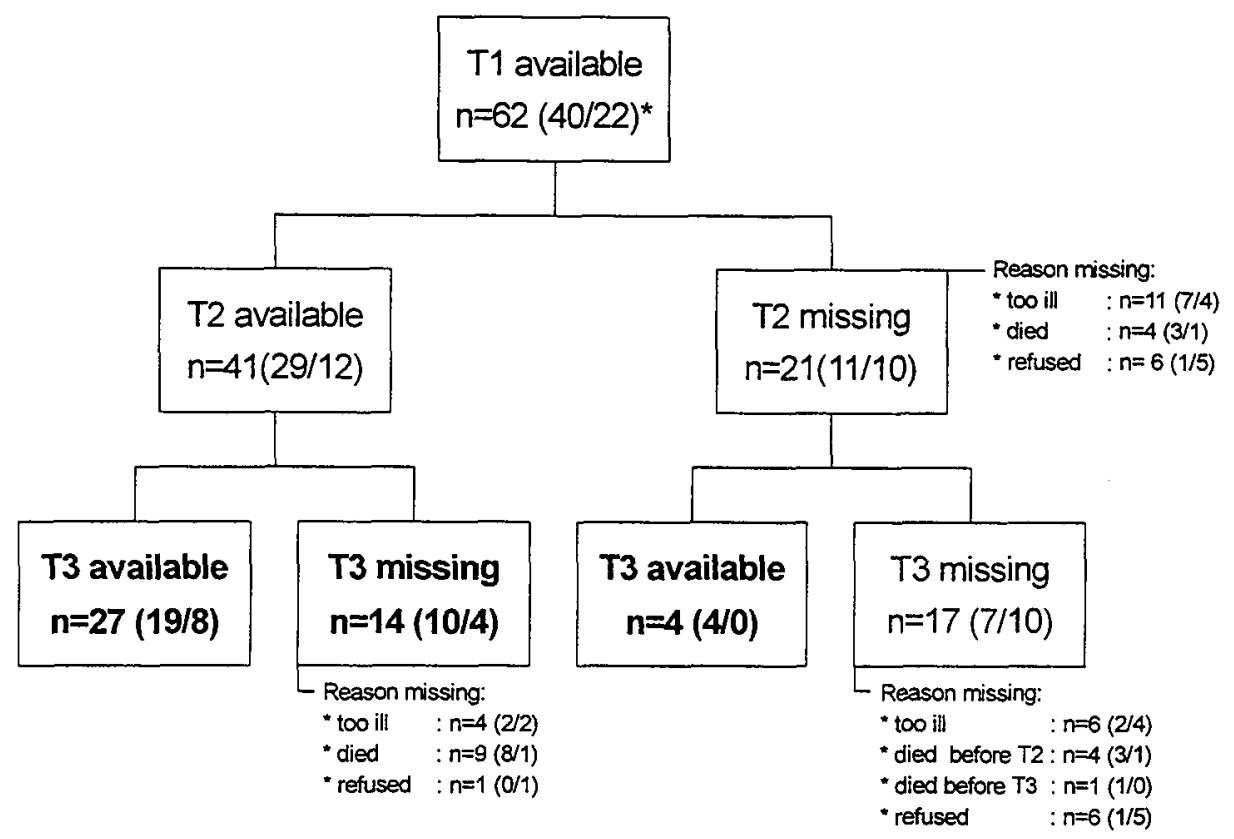

Figure 3 Number of, and reasons for, missing quality of life questionnaires

$\mathrm{n}=$ number of patients, ${ }^{*}=$ (number intervention group/number control group), only the patients who completed one or more of the post-intervention quality of life measurements (at T2 and/or T3) could be used in the quality of life analyses. These are the patients denoted in the boxes with bold letters.

\section{Outcome measures}

Patients in the intervention group showed significantly lower levels of re-hospitalization during the terminal phase of their illness compared to those patients in the control group (Table 2). In the sub-group of patients in which the effects of the intervention on the patient's quality of life could be evaluated, the same difference in re-hospitalization between the intervention and control group was observed. No significant confounders could be identified for this outcome measure. We therefore confined ourselves to a univariate comparison. 
Table 1 Initial patient characteristics

\begin{tabular}{|c|c|c|c|c|}
\hline \multicolumn{3}{|c|}{ Initial patient characteristics } & IG $(n=79)$ & CG $(n=37)$ \\
\hline \multicolumn{5}{|c|}{ Personal characteristics } \\
\hline \multicolumn{3}{|c|}{ age (mean age $\pm S D)$} & $64.6 \pm 10.9$ & $63.7 \pm 9.8$ \\
\hline \multicolumn{3}{|c|}{ gender ( $\%$ male $)$} & $59.5 \%$ & $62.2 \%$ \\
\hline \multicolumn{2}{|c|}{ socio-economic status: } & & $44.6 \%$ & $43.3 \%$ \\
\hline \multicolumn{3}{|c|}{ middle } & $37.5 \%$ & $20.0 \%$ \\
\hline \multicolumn{3}{|c|}{ high } & $17.9 \%$ & $36.7 \%$ \\
\hline \multicolumn{3}{|c|}{ living with partner } & $67.1 \%$ & $91.7 \% *$ \\
\hline \multicolumn{3}{|c|}{ belief (\% Roman-Catholic) } & $80.9 \%$ & $81.8 \%$ \\
\hline \multicolumn{3}{|c|}{ pre-morbid health status $\dagger$ (mean $\pm S D$ ) } & $10.7 \pm 4.6$ & $10.6 \pm 4.0$ \\
\hline \multirow{4}{*}{\multicolumn{2}{|c|}{ coping behavior $\neq$ (mean \pm SD): }} & problem solving & $13.6 \pm 2.7$ & $13.9 \pm 2.9$ \\
\hline & & seeking social support & $8.4 \pm 3.4$ & $11.4 \pm 4.2^{\star}$ \\
\hline & & palliative reaction & $4.4 \pm 1.4$ & $4.1 \pm 1.4$ \\
\hline & & avoidance & $5.6 \pm 2.1$ & $5.8 \pm 2.0$ \\
\hline \multicolumn{5}{|c|}{ Disease characteristics } \\
\hline \multirow[t]{5}{*}{ primary tumor: } & lung & & $29.1 \%$ & $35.1 \%$ \\
\hline & intestine & & $30.4 \%$ & $24.3 \%$ \\
\hline & breast & & $11.4 \%$ & $13.5 \%$ \\
\hline & other & & $29.1 \%$ & $17.1 \%$ \\
\hline & metastasi & & $73.1 \%$ & $75.0 \%$ \\
\hline \multirow[t]{4}{*}{ initial treatment: } & chemothe & & $17.7 \%$ & $35.3 \% *$ \\
\hline & operative & & $45.6 \%$ & $55.9 \%$ \\
\hline & radio-ther & & $41.8 \%$ & $38.2 \%$ \\
\hline & hormonal & & $6.3 \%$ & $11.8 \%$ \\
\hline \multirow[t]{4}{*}{ present treatment: } & chemothe & & $12.7 \%$ & $11.8 \%$ \\
\hline & operative & & $2.5 \%$ & $2.9 \%$ \\
\hline & radio-ther & & $10.1 \%$ & $8.8 \%$ \\
\hline & hormonal & & $3.8 \%$ & $2.9 \%$ \\
\hline \multicolumn{3}{|c|}{ duration of the disease in months (mean $\pm S D$ ) } & $16.0 \pm 23.0$ & $23.3 \pm 34.3$ \\
\hline \multicolumn{3}{|c|}{ patients having significant co-morbidity } & $41.0 \%$ & $45.7 \%$ \\
\hline \multicolumn{3}{|c|}{ Social network characteristics } & Mean $\pm S D$ & Mean $\pm S D$ \\
\hline \multicolumn{3}{|c|}{ number of persons in the household } & $.9 \pm .7$ & $1.3 \pm .8^{\star}$ \\
\hline \multicolumn{3}{|c|}{ number of women in the household } & $.5 \pm .6$ & $.8 \pm .7^{*}$ \\
\hline \multicolumn{3}{|c|}{ number of persons in the social network } & $6.8 \pm 4.0$ & $7.4 \pm 4.6$ \\
\hline \multicolumn{3}{|c|}{ number of nwm giving emotional support } & $5.2 \pm 3.5$ & $7.0 \pm 4.5$ \\
\hline \multicolumn{3}{|c|}{ number of nwm giving practical support } & $3.6 \pm 2.9$ & $5.0 \pm 3.7$ \\
\hline \multicolumn{3}{|c|}{ number of nwm giving support in dealing with the disease } & $5.7 \pm 3.8$ & $6.7 \pm 4.8$ \\
\hline \multicolumn{3}{|c|}{ total number of contacts with nwm per year } & $105.4 \pm 60.6$ & $112.0 \pm 61.4$ \\
\hline mean of emotional & upport per $r$ & & $3.1 \pm 1.0$ & $3.5 \pm .5$ \\
\hline mean of practical si & port per nv & & $2.4 \pm 1.2$ & $2.7 \pm 1.0$ \\
\hline mean of support in & ealing with & isease per nwm & $3.3 \pm .8$ & $3.4 \pm 1.0$ \\
\hline Primary outcome & easures & & & \\
\hline 'Physical functionin & at T1\| & & $.22 \pm 2.1$ & $-.56 \pm 2.1$ \\
\hline Psycho-social func & ning' at T1 & & $-.26 \pm 3.2$ & $.99 \pm 2.1$ \\
\hline
\end{tabular}




\section{Footnotes to table 1}

$I G=$ intervention group, $C G=$ control group, $n=$ number of patients, $S D=$ standard deviation, $\dagger=a$ higher level indicates a less favorable premorbid health status, $\ddagger=a$ higher level indicates a more frequent use of that specific coping behavior, Nwm = network members, $\S=a$ higher mean level indicates a higher level of experienced support per nwm, $\|=a$ higher level indicates a better functioning. For comparisons Student's $t$ tests were applied in cases of normally distributed variables, otherwise $x^{2}$ tests were applied. No significant differences between the 2 groups were found except where otherwise indicated: ${ }^{*}=p<.05$.

\section{Table 2 Results of the re-hospitalization analyses}

\begin{tabular}{ccc}
\hline & IG & CG \\
\hline Total group & $\mathrm{n}=79$ & $\mathrm{n}=37$ \\
\hline Days in hospital & $5.8 \pm 12.8$ & $11.5 \pm 17.1^{\star}$ \\
Survival (in days) & $101.2 \pm 141.5$ & $68.6 \pm 81.5 \mathrm{~ns}$ \\
\% of pat. who died at home & 80.8 & $64.9 \mathrm{~ns}$ \\
\hline "QOL" group & $\mathrm{n}=33$ & $\mathrm{n}=12$ \\
\hline Days in hospital & $7.0 \pm 12.1$ & $25.0 \pm 21.7^{*}$ \\
Survival (in days) & $143.0 \pm 162.7$ & $115.8 \pm 63.5 \mathrm{~ns}$ \\
\% of pat. who died at home & 71.9 & $58.3 \mathrm{~ns}$ \\
\hline
\end{tabular}

See table 1 . The values in the tables represent means $\pm S D$. pat. = patient, " $Q O L$ " group $=$ the sub-group of patients whose quality of life could be analysed. All statistical comparisons were performed using the Mann-Whitney $U$ test, except for the comparisons for place of death. Here, the $X^{2}$ test was used. ${ }^{*}=p<.01$, ns $=$ not significant.

Based on the aforementioned procedure, 4 potential confounders were identified in the relationship between the experimental condition and the quality of life outcome measures, i.e.: the patient having had radiotherapy in the past for palliative reasons; 'seeking social support' as a means of coping behaviour; the number of persons in the household; and the mean practical support per social network member. These were included as independent variables together with the pre-intervention quality of life value (value at $\mathrm{T} 1$ ) in the multiple regression analyses (Table 3 ). This showed that at T3 the intervention programme contributed significantly $(p=.048)$ towards a better 'physical' functioning. At $\mathrm{T} 2$ the same trend was observed $(p=.065)$.

Concerning the secondary outcome measures, we saw that more patients in the intervention group died at home ( $81 \%$ versus $65 \%$; difference not significant; $\mathrm{p}=.06$ ). The length of survival was higher in the intervention in the intervention group (Table 2), but again this difference was not significant. 


\section{Discussion}

To our knowledge this is the first controlled study investigating the effects of a transmural home care programme (based on a complementary approach to care with optimal communication channels between intra- and extramural health care organizations) on the incidence of re-hospitalization and terminal cancer patients' quality of life.

\section{Table 3 Results of the multiple regression analyses}

\begin{tabular}{lcccc}
\hline \multirow{2}{*}{ Independent variable } & PSF T2 & PSF T3 & PhF T2 & PhF T3 \\
\cline { 2 - 5 } & $\beta$ & $\beta$ & $\beta$ & $\beta$ \\
\hline Value of QOL OM at T1 & $.54 \dagger$ & $.44 \dagger$ & $.44 \dagger$ & $.34^{\star}$ \\
Prior RT for palliative reasons & - & - & - & $-.45^{\star}$ \\
Seeking social support & - & - & $.30^{*}$ & $.39 \dagger$ \\
N. of persons in the household & - & $-.43^{*}$ & - & $-.38^{*}$ \\
Mean practical support per nwm & $.33 \dagger$ & - & - & - \\
Intervention Group & $.09 \mathrm{~ns}$ & $-.21 \mathrm{~ns}$ & $.28 \mathrm{~ns}$ & $.29^{*}$ \\
\hline Adjusted R & $.50 \ddagger$ & $.37 \dagger$ & $.28 \dagger$ & $.57 \ddagger$ \\
Number of patients & 40 & 30 & 40 & 30 \\
F value & 13.51 & 6.79 & 4.52 & 8.71 \\
\hline
\end{tabular}

$\mathrm{PSF}=$ 'psycho-social functioning', $\mathrm{PhF}=$ 'Physical functioning', $\mathrm{QOL}=$ quality of life, $\mathrm{OM}$ = outcome measure, $\mathrm{RT}$ = radiotherapy, $\mathrm{N}$. = number, $\mathrm{nwm}=$ network member, $\mathrm{R}^{2}=$ coefficient of determination, $\beta=$ standardized regression weight, $n s=$ not significant, ${ }^{*}=p<.05, \dagger=p<.01, \ddagger=p<.001$.

Terminally ill cancer patients on this transmural intervention programme underwent significantly less re-hospitalization during the terminal phase of their illness (on average 5.8 days of hospitalization over a mean survival period of 101 days) when compared to those patients receiving standard care (on average 11.5 days over a mean survival period of 69 days; $\mathrm{p}<.01$ ). In this study we were unable to find any confounders in the relationship between the experimental condition and re-hospitalization. This indicates that this lower re-hospitalization was caused by the intervention itself. This was achieved without negatively influencing on the patient's quality of life. Our results even indicate a probable positive effect on the patients 'physical' quality of life, whilst the patient's 'psycho-social' quality of life remained unaffected by the intervention.

Eighty-one percent of the patients in the intervention group and 65 percent of the patients in the control group, eventually died at home. This percentage is considerably higher than that observed by Constantini in his palliative home care service study: i.e. $60 \% .{ }^{42} \mathrm{McWhinney}$ et al. only found $28 \%$ of his palliative home care team users died at home. ${ }^{22}$ The percentage of our patients dying at home is also substantially higher than 
those seen in the USA, where less than 50 percent of all people die at home. ${ }^{43}$

These results can be criticized on two aspects. Firstly, it may be argued that 'dropout' may have influenced the results on the quality of life analyses of this study. Dropout in the intervention and control group was relatively high, but this is not unusual in these kind of studies. ${ }^{2,44,45}$ Drop-out, however, was not significantly different between the 2 groups. Furthermore, the 'drop-out' patients matched the study patients for initial characteristics. Therefore, a selection bias due to drop-out is unlikely.

Secondly, one may question whether the disadvantages of a quasi-experimental design have been adequately accounted for. In view of this, it is important to recognize that the 2 different areas from which the patients in the intervention and control group were derived are comparable with respect to hospital accessibility and the availability of home care services. In the near future these 2 areas will even be merged into one city province. Furthermore, the 2 groups appeared to be fairly similar for initial patient characteristics. Nevertheless the data were checked for the presence of confounders and these were included (together with the pre-intervention value of the quality of life outcome measure) in the multiple regression analyses. Due to these precautions, the final results can be viewed as valid.

During the study both carers and patients became very enthusiastic about the intervention, introducing a possible contamination bias. Therefore, after 13 months, implementation of the programme on a large scale by the hospital could no longer be prevented. This possible contamination bias may affect the results of this study in that they may represent an underestimation of the real effect.

When reviewing the literature, no studies evaluating the effects of similar intervention programmes were found. Home care interventions that have been studied were developed and carried out by hospices ${ }^{15}$ or hospitals ${ }^{13,14,16}$ on their own. In other studies the effects of community nursing home care interventions were only compared to the absence of community care. ${ }^{46,47}$ The results of all these studies showed no consistency in the effects the interventions had on the quality of life for these patients, nor on their rehospitalization rate, although patients generally expressed greater satisfaction with the care they received. The different types of interventions and kinds of standard care offered to the control groups were probably responsible for these inconsistent findings. All these programmes were characterized by the introduction of additional patient home care facilities for patients whereas this transmural home care programme did not. In contrast to the other interventions, this programme was mainly focused on improving the coordination and communication between caregivers in order to enhance the continuity of care (the only additional home care facility that was introduced by our programme was the transmural home team; the help of this team, however, was seldomly, i.e. 8 times, requested). These aspects, i.e. adequate communication and cooperation between caregivers and optimal continuity in care may be more important in preventing hospitalizations and for maintaining the best possible quality of life for terminally ill cancer patients, rather than the introduction of extra home care facilities. 


\section{Practice implications}

In conclusion, this transmural home care intervention programme significantly reduced re-hospitalization of terminally ill cancer patients, while, at the same time, it also appeared to contribute positively to the patient's 'physical' quality of life. The introduction of measures to enhance coordination and cooperation of intra- and extramural care, seems to be an improvement compared to standard community care. Therefore, health care policies should encourage the future development of these transmural care programmes. 


\section{References}

1. Fraumeni JF, Hoover RN, Devesa SS, Kinlen LJ. Epidemiology of cancer. In: Devita VT, Hellman S, Rosenberg SA, eds. Cancer. Principles \& practice of oncology. Philadelphia: J.B. Lippincott, 1993;196-236.

2. Ahmedzai S. Quality of life research in the European palliative care setting. In: Osaba D, ed. Effect of cancer on quality of life. London: CRC Press, 1991;323-32.

3. Steering Committee on Future Health Care Scenarios. Cancer in the Netherlands: scenarios on cancer 1985-2000. Dordrecht: Kluwer Academic Publishers, 1988.

4. Macdonald $N$. The interface between oncology and palliative medicine. In: Doyle $D$, Hanks GWC, MacDonald N, eds. Oxford textbook of palliative medicine. Oxford: Oxford University Press, 1997;11-7.

5. van den Akker PAM, Tits MHL, Kok NM. Leven met de dood. Over terminale patienten en terminale zorg in Nederland [Living with death. Terminal patients and terminal care in The Netherlands]. Houten: Bohn Stafleu Van Loghum, 1994.

6. Field D, James N. Where and how people die. In: Clark D, ed. The future of palliative care. Buckingham: Open University Press, 1993;6-29.

7. Jones RV, Hansford J, Fiske J. Death from cancer at home: the carers' perspective. $B M J$ 1993;306:249-51.

8. Wilkes E. Dying now. Lancet $1984 ; \mathbf{i}: 950-2$.

9. O'Hare PA, Yost LS, McCorkle R. Strategies to improve continuity of care and decrease rehospitalization of cancer patients: a review. Cancer Invest 1993;11:140-58.

10. Conkling VK. Continuity of care issues for cancer patients and families. Cancer 1989;64:290-4.

11. Herd EB. Terminal care in a semi-rural area. Br J Gen Pract 1990;40:248-51.

12. Johansen $H$, Nair $C$, Bond $J$. Who goes to the hospital? An investigation of high users of hospital days. Health Rep 1994;6:253-77.

13. Hughes SL, Cummings J, Weaver F, Manheim L, Braun B, Conrad K. A randomized trial of the cost effectiveness of VA hospital-based home care for the terminally ill. Health Services Research 1992;26:801-17.

14. Cummings JEB, Hughes SL, Weaver FM, Manheim LM, Conrad KJ, Nash K, et al. Cost-effectiveness of Veterans Administration hospital-based home care. A randomized clinical trial. Arch Intern Med 1990;150:1274-80. 
15. Greer DS, Mor V, Morris JN, Sherwood S, Kidder D, Bimbaum H. An alternative in terminal care: results of the National Hospice Study. J Chronic Dis 1986;39:9-26.

16. Zimmer JG, Groth-Junker A, McCusker J. A randomized controlled study of a home health care team. Am J Public Health 1985;75:134-41.

17. Schrijvers AJP. Health and Health Care in the Netherlands. A critical Self-assessment by Dutch Experts in the Medical and health Sciences. Utrecht: De Tijdstroom B.V. 1997.

18. McWhinney IR. Caring for patients with cancer. Family physicians' role. Can Fam Physician $1994 ; 40: 16-9$.

19. Wood ML. Communication between cancer specialists and family doctors. Can Fam Physician 1993;39:49-57.

20. Nationale Raad voor de Volksgezondheid/College voor Ziekenhuisvoorzieningen. Transmurale somatische zorg [Transmural somatic care]. Zoetermeer: Nationale Raad voor de Volksgezondheid, 1995.

a 21. Commissie modernisering curatieve zorg. Gedeelde zorg; betere zorg [Shared care; better care]. Zoetermeer: Commissie Modernisering Curatieve Zorg, 1994.

22. McWhinney IR, Bass MJ, Orr V. Factors associated with location of death (home or hospital) of patients referred to a palliative care team. Can Med Assoc J 1995;152:361-7.

23. Brown D, Roberts JA, Elkins TE, Larson D, Hopkins M. Hard choices: the gynecologic cancer patient's end-of-life preferences. Gynecol Oncol 1994;55:355-62.

24. Townsend J, Frank AO, Fermont D, Dyer S, Karron O, Wallgrove A, et al. Terminal cancer care and patients' preference for place of death: a prospective study. BMJ 1990;301:415-7.

25. Cook TD, Campbell DT. Quasi-experimentation. Design \& analysis issues for field settings. Boston: Houghton Mifflin Company, 1979.

26. Polit DF, Hungler BP. Nursing research. Principles and methods. Philadelphia: J.B. Lippincott company, 1995.

27. Tabachnick BG, Fidell CS. Using multivariate statistics. New York: HarperCollins College Publishers, 1996.

28. de Witte LP. After the rehabilitation centre. A study into the course of functioning after discharge from rehabilitation [Thesis]. Amsterdam/Lisse: Swets \& Zeitlinger, 1991.

29. Scheurs PJG, Tellegen B, van de Willege G. Coping lijst [Health, stress and coping: The development of the Utrecht Coping Scale]. Gedrag 1984;12:101-17. 
30. Furer JW, Konig-Zahn C, Tax B. Het meten van gezondheidstoestand. Deel 1 [The measurement of health state. Part 1]. Assen: Van Gorcum, 1995.

31. Husrisson EC. Visual analogue scales. In: Melzack P, ed. Measurement and assessment. New York: Raven Press, 1983;

32. de Haes JCJM, van Knippenberg FCE, Neijt JP. Measuring psychological and physical distress in cancer patients: structure and application of the Rotterdam Symptom Checklist. $\mathrm{Br}$ $J$ Cancer 1990;62:1034-8.

33. Bruin AF, Diederiks JPM, de Witte LP, Stevens FCJ, Philipsen H. The development of a short generic version of the Sickness Impact Profile. J Clin Epidemiol 1994;47:407-18.

34. de Witte L, Jacobs $H$, van der Horst F, Luttik A, Joosten J, Philipsen H. De waarde van de Sickness Impact Profile als maat voor het functioneren van patienten [The value of the Sickness Impact Profile as a measure for functioning of patients]. Gezondheid \& Samenleving 1987;8:120-7.

35. van der Ploeg HM. Validatie van de Zelf-Beoordelings-Vragenlijst [Validation of the Dutch version of the Spielberger State-Trait-Anxiety Inventory]. Ned Tijdschr Psychologie 1980;35:243-9.

36. de Jong-Gierveld J. Eenzaamheid: een meersporig onderzoek [Loneliness: a diverse investigation]. Deventer: Van Loghum Slaterus, 1984.

37. de Jong-Gierveld J, Tilburg T. Manual of the loneliness scale. Amsterdam: Dep. Social Research Methodology, Free University, 1990.

38. de Jong-Gierveld J, Kamphuis F. The development of a rash-type Ioneliness scale. Appl Psychol Measurement 1985;9:289-99.

39. Andrews FM, Witchey SB. Social indicators of well-being. Americans' perceptions of life quality. New York: Plenum Press, 1976.

40. de Haes JCJM. Kwaliteit van leven van kanker patienten [Quality of life of cancer patients]. Amsterdam/Lisse: Swets \& Zeitlinger, 1988.

41. Armitage P, Berry G. Statistical methods in clinical research. Oxford: Blackwell scientific publications, 1987.

42. Costantini MH, Carnoirano E, Maddedu L, Bruzzi P, Verganelli E, Henriquet F. Palliative home care and place of death among cancer patient: a population based study. Palliat Med 1993;7:323-31.

43. National Center for Health Statistics. Vital statistics of the United States, 1991. v. 2. Mortality. Part A, section I. Washington: US Gov Pr Office, 1996. 
Chapter 4

44. Osaba $\mathrm{D}$. Lessons learned from measuring health related quality of life in oncology. $J$ Clin Oncol 1994;12:608-16.

45. McWhinney IR, Bass MJ, Donner A. Evaluation of a palliative care service: problems and pitfalls. BMJ 1994;309:1340-2.

46. McCorkle RG, Benoliel JQ, Donaldson G, Georgiadou F, Moinpour C, Goodell B. A randomized clinical trial of home nursing care for lung cancer patients. Cancer 1989;64:1375-82.

- 47. McCorkle R, Jepson C, Malone D, Lusk E, Braitman L, Buhler-Wilkerson K, et al. The impact of posthospital home care on patients with cancer. Research in Nursing and Health $1994 ; 17: 243-51$. 


\section{Appendix}

Results of principal component analysis with orthogonal rotation performed on the patient's quality of life outcome measures

\begin{tabular}{|c|c|c|c|c|c|c|}
\hline \multirow{2}{*}{$\begin{array}{l}\text { Time-point } \\
\text { Factor }\end{array}$} & \multicolumn{2}{|c|}{ T1 } & \multicolumn{2}{|c|}{$\mathrm{T} 2$} & \multicolumn{2}{|c|}{ T3 } \\
\hline & 1 & II & 1 & $\|$ & 1 & $\|$ \\
\hline$E V / \%$ of variance & $3.1 / 44.6$ & $1.1 / 15.8$ & $1.6 / 22.5$ & $2.6 / 37.2$ & $1.4 / 19.5$ & $3.2 / 45.5$ \\
\hline Outcome measure & & & Factor & oadings & & \\
\hline SIP & .40 & .24 & .79 & -.33 & .79 & .04 \\
\hline Somatic complaints & .77 & .16 & .73 & .19 & .83 & .19 \\
\hline Pain & .78 & -.06 & .56 & .28 & .51 & .20 \\
\hline General well being & .45 & .64 & .09 & .67 & .44 & .66 \\
\hline Psychol. complaints & .61 & .51 & .53 & .65 & .40 & .77 \\
\hline Fear & .52 & .68 & .36 & .79 & .35 & .84 \\
\hline Loneliness & -.14 & .88 & .33 & .68 & -.37 & .74 \\
\hline
\end{tabular}

T1-T3: see text, EV = Eigenvalue; SIP = Sickness Impact Profile; psychol. $=$ psychological.

Within-group comparison of the means of 'Physical' (PF) and 'Psychosocial Functioning' (PSF) 1 week before (T1) with these outcome measures 1 week (T2) and 4 weeks (T3) after discharge from hospital.

\begin{tabular}{|c|c|c|c|c|c|c|c|c|}
\hline & \multicolumn{4}{|c|}{ Intervention group } & \multicolumn{4}{|c|}{ Control group } \\
\hline & $\mathrm{T} 1$ & $\mathrm{~T} 2$ & T1 & T3 & T1 & $T 2$ & T1 & T3 \\
\hline & \multicolumn{2}{|c|}{$(n=28)$} & \multicolumn{2}{|c|}{$(n=22)$} & \multicolumn{2}{|c|}{$(n=12)$} & \multicolumn{2}{|c|}{$(n=8)$} \\
\hline PSF* $^{\star}$ & .13 & $2.03 t$ & -.17 & .60 & .00 & $1.85 \ddagger$ & .13 & .60 \\
\hline $\mathrm{PF}^{\star}$ & .16 & .68 & .12 & .36 & .50 & .95 & -.39 & -.65 \\
\hline
\end{tabular}

T1-T3: see text, $\mathrm{n}=$ number of patients being compared, ${ }^{*}=\mathbf{a}$ higher value indicates $\mathbf{a}$ better functioning, $\ddagger=p<0.05, \dagger=p<0.01$ (Wilcoxon's test for paired samples). 


\section{CHAPTER}

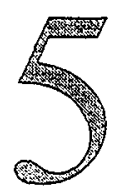

\section{Transmural care of terminal cancer patients: Effects on the quality of life of direct caregivers}

Accepted as:

Smeenk FWJM, de Witte LP, van Haastregt JCM, Schipper RM, Biezemans HPH, Crebolder HFJM. Transmural care of terminal cancer patients: Effects on the quality of life of direct caregivers. Nurs Res 1997. 


\section{Abstract}

Background - The burden of caring for terminal cancer patients has a negative effect on the informal caregivers' quality of life.

Objectives - To investigate the effects of a transmural home care intervention program for terminal cancer patients on the direct caregivers' (the patient's principal informal caregiver) quality of life, compared with standard care programs. The intervention program intended to optimize the cooperation and coordination between the intramural and extramural health care organizations (transmural care).

Methods - Direct caregivers of terminal cancer patients (estimated prognosis of less than 6 months) could be included in this quasi-experimental study. The direct caregivers' quality of life was measured in a multi-dimensional way 1 week before (T1), 1 week after (T2) and 4 weeks after (T3) the patient's discharge from hospital (discharge being the starting point of the intervention), then again at 3 months after the patient's death (T4). Factor analyses on the four outcome measures yielded one factor. This was considered the primary outcome measure and was named the Overall Quality of Life Index (OQOLI).

Results - Multiple regression analyses showed that the intervention contributed significantly positively to the direct caregivers' OQOLI at $\mathrm{T} 2(\beta=.30 ; \mathrm{p} \leq .05)$ and T4 $(\beta=.28 ; \mathrm{p} \leq .05)$, compared with standard care.

Conclusion - Transmural care forms a significantly positive contribution to the OQOLI of direct caregivers of terminal cancer patients 1 week after the patient's discharge from hospital and 3 months after the patient's death. Good terminal care also appears to be important for direct caregivers as well, with respect to perceived quality of life. 


\section{Introduction}

In developed countries cancer is one of the main causes of death. ${ }^{1}$ Fifty percent of all cancer patients cannot be cured. ${ }^{2,3}$ The quality of life of these incurable cancer patients will eventually diminish. ${ }^{4}$ In their terminal phase they are often readmitted to hospital., A main reason for this is that the direct caregiver is no longer able to care for the patient. $^{7}$ Other reasons are that the community care teams are unable to adequately alleviate symptoms in certain circumstances, or that there is inadequate communication between the different professional caregivers. ${ }^{8-10}$

The same high re-admission rate of these patients was also observed in The Netherlands, despite the availability of a primary care team for every patient. "This primary care team consists of a general practitioner (available for consultation 24 hours a day), a community nurse (for 24 hours if necessary), family aid for (professional) assistance in the household if necessary, and a service providing special equipment e.g. special beds, equipment for epidural analgesia et cetera. Furthermore, most patients are regularly checked by their hospital based specialist.

To overcome these problems home care programs based in hospices or hospitals and domiciliary care teams have been developed in Britain and the United States. ${ }^{4.12}$ Until now, most studies in this field have investigated the effects of these home care programs on the quality of life of the patients themselves or on possible cost savings. ${ }^{12-15}$ A search in the MEDLINE database yielded few studies addressing the effect of home care programs for terminal cancer patients on the quality of life of the patients' direct caregivers. ${ }^{16-18}$ None of these studies were both prospective and controlled.

In order to be able to provide better supportive care at home for these patients and their direct caregivers (i.e. the informal caregiver who is presumed to be the patient's principal caregiver and will be the patient's partner in the majority of cases), an intervention program was developed. Its main objective was to offer care to the patient that was specially tailored to meet his or her individual needs, and offered by professional caregivers from primary and hospital teams. The intervention program intended to maximize continuity of care by optimizing the cooperation, coordination and communication between the various professional carers. With this care setting, in contrast to the traditional home care programs, the community based primary health care team maintained responsibility for the patient but received specifically designed backup from the hospital team. This kind of care was named transmural care in The Netherlands. ${ }^{19}$ The development of this kind of care at a regional level was highly recommended by the Dutch government. ${ }^{20}$

It was hypothesized that this transmural care may lead to a better quality of life for direct caregivers of terminal cancer patients. 


\section{Methods}

\section{Design of the study}

Because of practical and ethical reasons, the design was quasi experimental. ${ }^{21-23}$ Direct caregivers of patients fulfilling the inclusion criteria and living in Eindhoven, The Netherlands, were allocated to the intervention group and those living in the urban surroundings of Eindhoven were allocated to the control group. After the patients' discharge from the hospital (i.e. the start of the intervention), patients and their direct caregivers in both groups received, as usual, the standard care available in The Netherlands. ${ }^{11}$ In addition to this care, patients and their direct caregivers allocated to the intervention group were offered the intervention program.

Quality of life of the direct caregivers was measured one week before (T1), one week after (T2), and 4 weeks (T3) after the patient's discharge from the hospital as well as 3 months after the patient had died (T4).

Considering the design of the study, it has to be recognized that these 2 areas are very similar. Eindhoven is a city of 200,000 inhabitants with a highly urbanized surrounding area. About 200,000 people live in these urbanized surroundings. The maximum traveling distance to our hospital for these people is about $30 \mathrm{~km}$ (20 miles), making hospital access easy for everyone in the study. These areas are in fact so urbanized that together they form 1 big city. The Dutch government has therefore recently decided to unite Eindhoven and its surrounding municipalities into one city province. The availability of home care services was also similar in both groups, and a pilot study using the data base of the Comprehensive Cancer Centre South did not show any significant differences in the incidence of the various cancer types, mean age, and gender of cancer patients in these 2 populations.

The study was approved by the Medical Ethical Committee of the hospital.

\section{The intervention program}

The transmural home care intervention program consisted of 4 main elements: a specialist nurse coordinator, a 24-hour telephone service in the hospital with access to $a$ transmural home team, a collaborative home care dossier (case file), and protocols designed for specific care.

\section{The specialist nurse coordinator}

The specialist nurse coordinator is the key person in this intervention program. On request of the treating specialist, she prepares the necessary patient discharge arrangements. Patient's wishes and (care) needs are assessed by her as well as the informal caregivers' capacity in supporting the patient. Taking these findings into account she plans and arranges home support by professional caregivers, which will naturally be tailored to meet patient's individual needs. For this to be successful, she needs to have daily contact with a wide variety of caregivers, from medical specialists to home helpers. She monitors the care provision process and tracks down and solves 
possible defaults or shortcomings.

The 24-hour telephone service and transmural home team (See Figure 1)

A 24-hour telephone consultation service was installed on the hospital's multidisciplinary oncology ward. This service is manned by nurses from the ward, who were specially trained in administering telephone assistance to patients. In the event of problems arising at home, this service can be contacted for advice 24 hour a day by telephone via a direct line (no operator delays or interference). For any medical questions a specialist consultant can be contacted using the service. To give adequate advise, a copy of the patient's home care dossier, containing all the essential medical and nursing information, is at hand. Thus, continuity of care is guaranteed.

If specific nursing problems can not be solved by the primary care team, support is provided by trained nurses from the hospital's transmural home team, on request of the patient's general practitioner. In general, problems that the team has to solve involve the high-technology devices often used in treatment. The home team consists of nurses from the hospital's casualty and day care departments. During on call hours they can be contacted by semaphone.

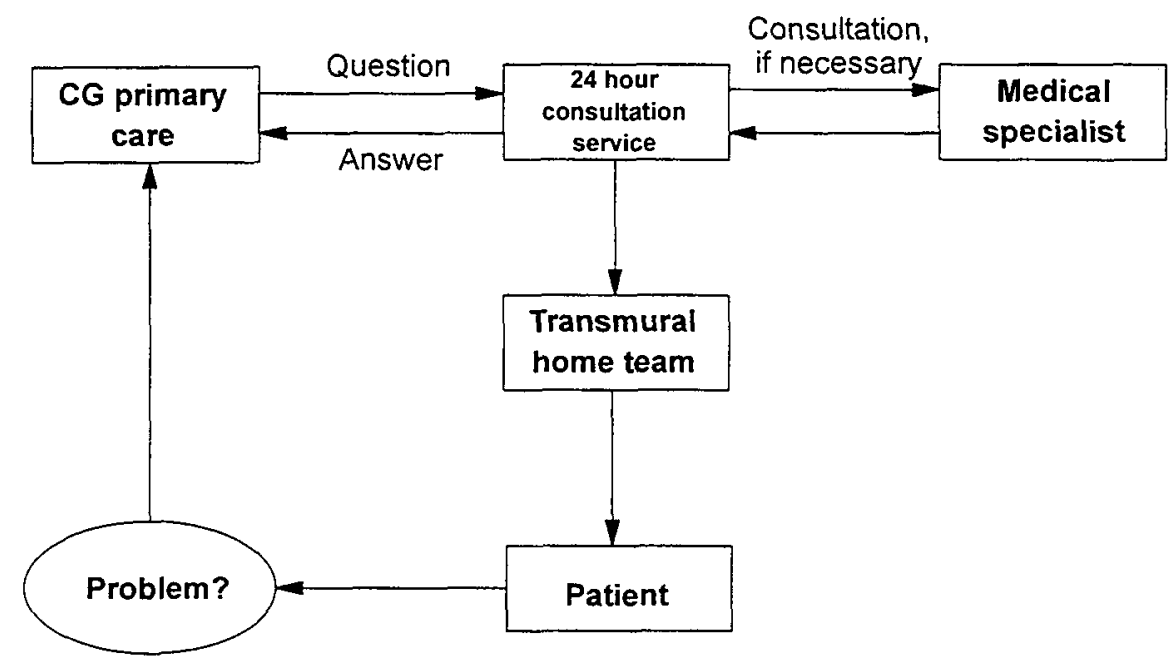

Figure 1 Organization structure of the 24-hour telephone consultation service
and the transmural home team ( $C G=$ caregiver)

The home care dossier

The home care dossier was developed to improve communication between caregivers and enhance the coordination of care. Caregivers from primary and hospital care teams were asked to collaborate in reporting their findings and actions undertaken. Hereby all caregivers are informed about findings and care plans. The dossier consists of the following items: an informed consent; a list of those caregivers involved in the care with 
this patient; a preliminary discharge report for the general practitioner; a nursing transfer report for the community nurse; a transfer report for home helpers dealing mainly the selfcare possibilities of the patient or the support available from his informal caregivers; the medication list; a dietician's report; and a multidisciplinary report. In this last section caregivers are asked to report their main findings and changes to their treatment/care plan. Where appropriate, care protocols are added to this file. Patients and informal caregivers also have access to the dossier. Patients themselves act as the actual trustees of their dossier.

\section{The care protocols}

The care protocols are intended to improve the quality of the care provided at home. These protocols are developed by a multidisciplinary team. The protocols standardize and support caregivers when using specific skills and actions. Protocols that were introduced are: intravenous therapy, epidural and spinal pain relief, and the pharmaceutical trajectory. In the protocol for the pharmaceutical trajectory the delivery arrangements of medications and sterile medical aid supplies are recorded.

As can be seen from Figure 1, the intervention does not interfere with existing health care organizational structures. So, for patients being admitted to hospital the medical consultant specialist retains responsibility, and for patients at home their general practitioner is responsible.

\section{Direct caregivers and the patients}

The criteria for inclusion in this study were related to the characteristics of the patient to be taken care of. The patient had to have a diagnosis of cancer, an estimated prognosis of less than 6 months, to be at least 18 years of age, to be fully informed of his or her diagnosis, and to be admitted to the hospitals' multidisciplinary oncology unit.

Patients and their direct caregivers were included from January 1994 till the first of February 1995.

\section{Initial direct caregiver and patient characteristics}

In the study the patient was the primary subject under observation. Therefore, a wide spectrum of initial patient characteristics were collected: data concerning the disease and its treatment, the patients' social network and social support, ${ }^{24}$ the sociodemographic characteristics and personal characteristics (coping as measured by the short version of the Utrecht Coping List, ${ }^{25}$ premorbid health status by the General Health Perception Questionnaire, ${ }^{26}$ and faith) were collected upon entry to the study.

The following characteristics were collected regarding the direct caregiver: age, gender, relationship with the patient, and professional status. It was assumed that the characteristics of the social economic status and social network of the direct caregivers would be comparable with those of the patients. 


\section{Primary outcome measures}

The quality of life of the direct caregiver was determined in a multidimensional way, using four different instruments, and comprised the following dimensions: daily functioning (Sickness Impact Profile [SIP] 68:27.29 The items dealing with somatic autonomy and motor control were not included because these items are not relevant for healthy persons), fear (Spielberger State Trait Anxiety Inventory) ${ }^{30}$ loneliness, ${ }^{31-33}$ and general well-being. ${ }^{34,35}$ All variables were obtained by two trained interviewers using structured questionnaires. These interviewers were blinded to the study design.

To yield an aggregate score for the quality of life measurements, principal component analyses with orthogonal rotation (method varimax) were performed at each assessment stage. ${ }^{23}$ Each analysis extracted 1 factor that was highly loaded by all four outcome measures. The sum of the standardized scores of the four outcome measures loading high on this factor was used as the primary outcome measure and was named Overall Quality Of Life Index (OQOLI). In all standardization procedures the mean scores and standard deviations of the four individual quality-of-life outcome measures obtained at $\mathrm{Tl}$ were used.

\section{Results}

\section{Direct caregivers}

Seventy nine direct caregivers were included in the intervention group and 37 in the control group. Sixty four direct caregivers ( 41 from the intervention and 23 from the control group; no significant difference) did not perform the pre-intervention quality-oflife assessment, mainly because they found it too burdensome. Of the remaining 52 direct caregivers, 7 (4 from the intervention and 3 from the control group; no significant difference) dropped-out, again mainly because they felt too burdened to complete the questionnaires (Figure 2). No significant differences could be found when comparing the drop-outs to the direct caregivers who could be evaluated on their initial characteristics.

The initial direct caregiver characteristics displayed in Table 1 and the initial patient characteristics shown in Table 2 demonstrate that both groups were fairly comparable. Tests being used for these comparisons were the student's $t$ test for those continuous variables that showed a normal distribution (indicated in the Table by the presentation of the SD) and the $\chi^{2}$ test for all other variables.

Before assessing whether the intervention contributed positively to the direct caregivers' $O Q O L I$, a search for possible confounders in the relationship between the experimental condition and this outcome measure was performed. For this, the correlations between the initial direct caregiver/patient characteristics and the outcome measure were assessed. Initial direct caregiver and patient characteristics that were significantly related to this outcome measure and that also showed a significant difference between the intervention and control group at $\mathrm{Tl}$, were considered as potential confounders. To prevent missing any potential confounders, a conservative significance level of $p \leq .10$ 


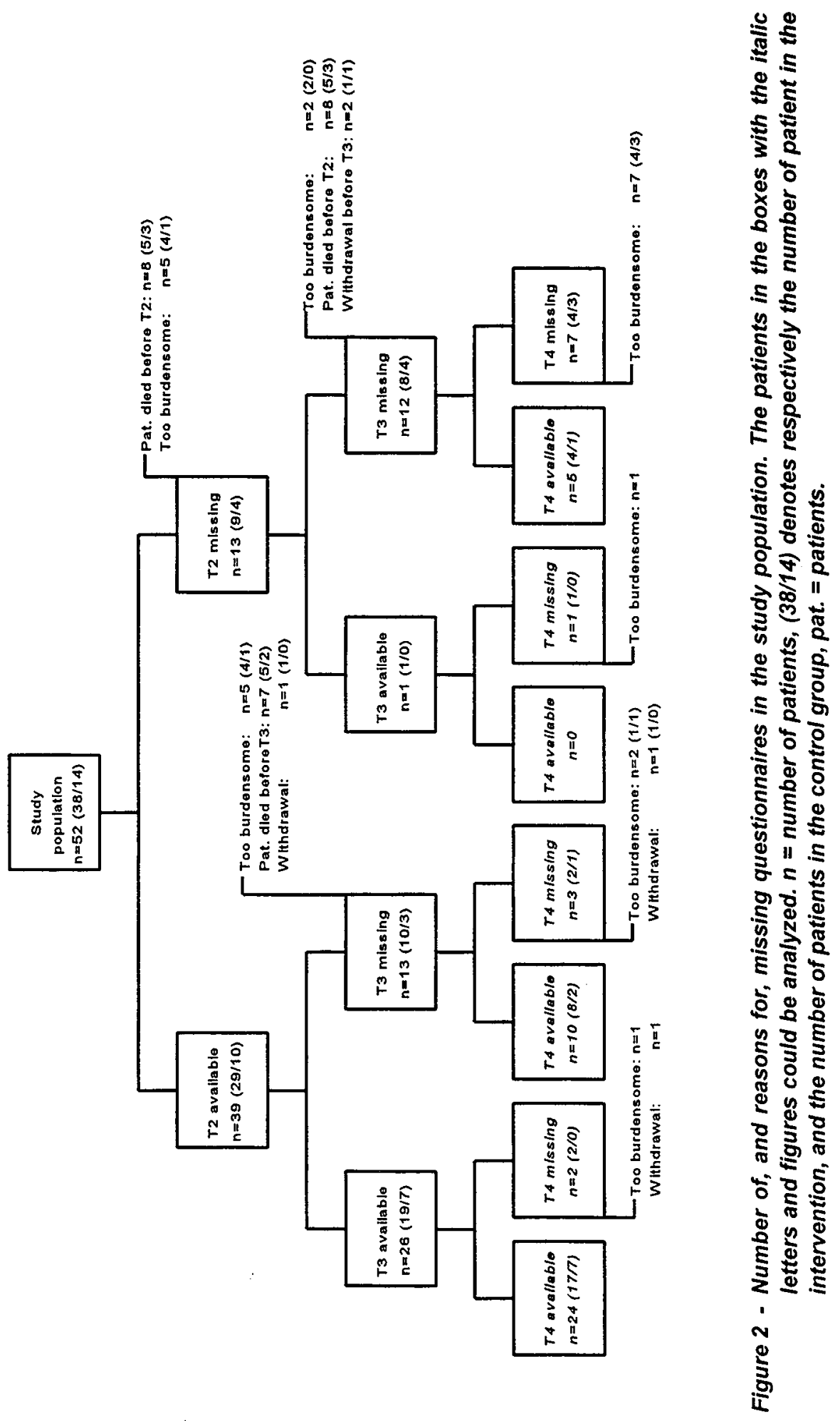


Table 1 Initial direct caregiver characteristics

\begin{tabular}{|c|c|c|c|}
\hline \multicolumn{2}{|c|}{ Direct Caregiver (DCG) characteristics } & \multirow{2}{*}{$\begin{array}{c}\text { IG } \\
N=34 \\
\text { Mean } \pm \text { SD }\end{array}$} & \multirow{2}{*}{$\begin{array}{c}C G \\
N=11 \\
\text { Mean } \pm \text { SD }\end{array}$} \\
\hline & & & \\
\hline Age & & $54.6 \pm 14.4$ & $61.2 \pm 10.7$ \\
\hline Gender ( $\%$ male) & & 29.4 & 36.4 \\
\hline \multicolumn{2}{|c|}{ Relationship with patient: \% partner } & 70.6 & $100.0^{\star}$ \\
\hline & $\%$ child & 14.7 & .0 \\
\hline & $\%$ sibling & 2.9 & .0 \\
\hline & $\%$ son/daughter in law & 2.9 & .0 \\
\hline & $\%$ friend & 8.8 & .0 \\
\hline \multirow[t]{3}{*}{ Professional status $\uparrow:$} & $\%$ low & 25.0 & 20.0 \\
\hline & $\%$ middle & 75.0 & 60.0 \\
\hline & $\%$ high & .0 & 20.0 \\
\hline OQOLI $\ddagger$ at $\mathrm{T} 1$ & & $-.19 \pm 3.0$ & $.65 \pm 3.4$ \\
\hline
\end{tabular}

IG = intervention group, $C G=$ control group, $N=$ number of patients, $S D=$ standard deviation, $\dagger=$ only data available of caregivers who are the patient's partner (intervention group $=24 \mathrm{DCGs}$, control group $=11 \mathrm{DCGs}), \mathrm{OQOLI}=$ overall quality of life index $\neq$ a higher value indicates a better OQOLI. No significant differences between the 2 groups were found except where otherwise indicated: ${ }^{*}=p<.05$.

was used. Eventually 2 potential confounders were identified: The patient initially being treated with chemotherapy and the direct caregiver also being the patient's partner.

To assess whether the intervention contributed positively to the direct caregivers' $O Q O L I$, hierarchical backward multiple regression analyses were performed on this outcome measure. ${ }^{23}$ To control for initial differences in the direct caregivers' OQOLI and the potentially confounding factors, these were added to the experimental condition as independent variables in the regression analyses. The final results of these analyses are depicted in Table 3, showing that the intervention contributed positively to the direct caregivers' $O Q O L I$ at $\mathrm{T} 2$ and $\mathrm{T} 4$.

In looking for evidence for those dimensions of the direct caregiver's quality of life that could have caused these positive effects, the means of the 4 separate dimensions used to construct the OQOLI, as well as the means of this OQOLI at the subsequent time assessments are presented in Table 4, which shows that the positive contribution of the intervention to the $O Q O L I$ at $\mathrm{T} 2$ was probably caused by improvements on the fear, general well-being, and loneliness scales, whereas at $\mathrm{T} 4$ this was probably caused by improvements on the SIP and fear scales. 
Table 2 Initial characteristics of the patient the direct caregiver had to care for

\begin{tabular}{|c|c|c|c|}
\hline \multirow{2}{*}{\multicolumn{2}{|c|}{ Patient characteristics }} & $\begin{array}{c}\text { IG } \\
N=34\end{array}$ & $\begin{array}{c}C G \\
N=11\end{array}$ \\
\hline & & Mean \pm SD & Mean \pm SD \\
\hline \multicolumn{4}{|c|}{ Personal characteristics } \\
\hline \multicolumn{2}{|c|}{ age } & $63.1 \pm 11.2$ & $62.1 \pm 8.1$ \\
\hline \multicolumn{2}{|c|}{ gender ( $\%$ male) } & 50.0 & 63.6 \\
\hline \multicolumn{2}{|c|}{ social economic status: \% low } & 41.4 & 50.0 \\
\hline \multicolumn{2}{|c|}{$\%$ middle } & 37.9 & 20.0 \\
\hline \multicolumn{2}{|c|}{$\%$ high } & 20.7 & 30.0 \\
\hline \multicolumn{2}{|c|}{$\%$ of patients living with partner } & 70.6 & $100.0^{*}$ \\
\hline \multicolumn{2}{|c|}{ pre-morbid statust: } & $11.0 \pm 5.2$ & $9.6 \pm 3.2$ \\
\hline \multirow[t]{4}{*}{ coping behavior } & problem solving & $13.5 \pm 2.8$ & $13.1 \pm 2.5$ \\
\hline & seeking social support & $8.4 \pm 3.4$ & $10.3 \pm 4.0$ \\
\hline & palliative reaction & $4.6 \pm 1.3$ & $4.0 \pm .7$ \\
\hline & avoidance & $5.4 \pm 2.2$ & $6.0 \pm 2.1$ \\
\hline \multicolumn{4}{|c|}{ Disease characteristics } \\
\hline \multirow[t]{4}{*}{ primary tumor: } & $\%$ lung & 26.5 & 54.5 \\
\hline & $\%$ intestine & 32.4 & $.0^{*}$ \\
\hline & $\%$ breast & 17.6 & 18.2 \\
\hline & $\%$ other & 23.5 & 27.3 \\
\hline \multicolumn{2}{|c|}{$\%$ with metastasis } & 79.4 & 90.0 \\
\hline \multicolumn{2}{|c|}{$\%$ chemotherapy (as initial treatment) } & 8.8 & 30.0 \\
\hline \multicolumn{2}{|c|}{$\%$ radiotherapy (as initial treatment) } & 41.2 & 60.0 \\
\hline \multicolumn{2}{|c|}{$\%$ surgical therapy (as initial treatment) } & 52.9 & 50.0 \\
\hline \multicolumn{2}{|c|}{$\%$ hormonal therapy (as initial treatment) } & 11.4 & 7.1 \\
\hline \multicolumn{2}{|c|}{$\%$ chemotherapy (at present) } & 9.1 & 14.3 \\
\hline \multicolumn{2}{|c|}{$\%$ radiotherapy (at present) } & 11.4 & 10.0 \\
\hline \multicolumn{2}{|c|}{$\%$ surgical therapy (at present) } & 4.5 & 1.4 \\
\hline \multicolumn{2}{|c|}{$\%$ hormonal therapy (at present) } & .0 & 7.1 \\
\hline \multicolumn{2}{|c|}{ duration of the disease in months } & $18.1 \pm 25.8$ & $29.0 \pm 51.2$ \\
\hline \multicolumn{2}{|c|}{$\%$ of patients having significant co-morbidity } & 33.3 & 47.8 \\
\hline \multicolumn{4}{|c|}{ Social network characteristics } \\
\hline \multicolumn{2}{|c|}{ number of persons in the household } & $.7 \pm .5$ & $1.2 \pm .4^{\star}$ \\
\hline \multicolumn{2}{|c|}{ number of women in the household } & $.4 \pm .5$ & $.6 \pm .5$ \\
\hline \multicolumn{2}{|c|}{ number of persons in the social network } & $7.0 \pm 4.1$ & $6.7 \pm 4.9$ \\
\hline number of nwm & giving emotional support & $5.2 \pm 3.3$ & $5.9 \pm 4.7$ \\
\hline number of nwm & giving practical support & $4.1 \pm 3.4$ & $4.5 \pm 3.2$ \\
\hline number of nwm & giving support in dealing with the disease & $5.9 \pm 4.0$ & $5.6 \pm 5.1$ \\
\hline experienced em & totional support§ & $27.1 \pm 14.2$ & $27.4 \pm 18.9$ \\
\hline experienced pra & actical support & $22.8 \pm 12.9$ & $24.6 \pm 15.4$ \\
\hline experienced sup & pport in dealing with the disease & $29.4 \pm 15.7$ & $28.8 \pm 20.6$ \\
\hline number of conta & acts with persons in the network per year & $106.2 \pm 67.0$ & $120.9 \pm 82.7$ \\
\hline
\end{tabular}




\section{Footnotes to table 2}

See table $1, t=$ a higher level indicates a less favorable premorbid status, $\ddagger=$ a higher level indicates a more frequent use of that specific coping behavior, $\S=$ a higher level indicates a higher level of experienced support, nwm $=$ network members. No significant differences between groups were found except where otherwise indicated: ${ }^{\star}=p<.05$.

Table 3 Results of the multiple regression analyses

\begin{tabular}{|c|c|c|c|}
\hline \multirow{2}{*}{ Independent variable } & OQOLI at T2 & OQOLI at T3 & OQOLI at T4 \\
\hline & $\beta$ & $\beta$ & $\beta$ \\
\hline Value of OQOLI at T1 & $.62^{*}$ & $.66^{\star}$ & $.53^{\star}$ \\
\hline Initial treatment CT & - & - & - \\
\hline DCG partner of patient & - & - & - \\
\hline Intervention & $.30 \dagger$ & $.14 \mathrm{~ns}$ & $.28 t$ \\
\hline Adjusted $\mathrm{R}^{2}$ & $.40^{\star}$ & $.40 \ddagger$ & $.30^{*}$ \\
\hline$N$ & 39 & 27 & 37 \\
\hline F value & 13.14 & 9.17 & 9.07 \\
\hline
\end{tabular}

See table 1, T1-T4 see text. CT = chemotherapy, DCG = direct caregiver, $R^{2}=$ coefficient of determination, $\beta=$ standardized regression weight, $n s=$ not significant, $\dagger=p \leq$ $.05,{ }^{*}=p \leq .001, \neq=p \leq .01$.

Table 4 Mean score development of the means of the 4 separate quality of life dimensions and the OQOLI

\begin{tabular}{|c|c|c|c|c|c|c|c|c|}
\hline \multirow{3}{*}{$\begin{array}{l}\text { Group } \\
\text { Time point } \\
\mathbf{N}\end{array}$} & \multicolumn{4}{|c|}{ Intervention Group } & \multicolumn{4}{|c|}{ Control Group } \\
\hline & T1 & $T 2$ & T3 & T4 & T1 & $\mathrm{T} 2$ & T3 & T4 \\
\hline & 29 & 29 & 20 & 27 & 10 & 10 & 7 & 10 \\
\hline Loneliness§ & 2.3 & $1.0 t$ & 2.3 & 2.1 & 1.8 & 1.1 & 1.7 & 3.4 \\
\hline Fear§ & 23.9 & $19.5+$ & $20.1 \dagger$ & $19.0 \ddagger$ & 22.2 & 20.4 & 21.9 & 20.6 \\
\hline SIP§ & 9.1 & 8.7 & $7.3^{*}$ & $2.8 \ddagger$ & 8.6 & 11.6 & 10.7 & 6.9 \\
\hline Well being & 8.9 & $10.9 \dagger$ & 9.8 & 9.2 & 9.6 & 10.3 & 11.0 & 8.0 \\
\hline OQOLIII & .15 & $2.1 \ddagger$ & $1.4^{*}$ & $2.2 \dagger$ & .27 & .34 & .09 & -.39 \\
\hline
\end{tabular}

See table 1 and $3 . \S=$ a higher score represents a less favorable outcome, $\|=$ a higher score represents a more favorable outcome. The direct caregiver's scores at T2, T3 and T4 were compared with their preintervention scores ( $\mathrm{T} 1 \mathrm{scores}$ ) by using the Wilcoxon Signed Rank Test. Differences were not significant, except where otherwise indicated: * $=p \leq .05 ; \dagger=p \leq .01 ; \ddagger=p \leq .001$. 


\section{Discussion}

In this study the effects of an intervention program on the quality of life of direct caregivers of terminally ill cancer patients was investigated. The intervention program was characterized by measures being taken to enhance the coordination and cooperation between intramural and extramural health care organizations. Emphasis was put on good communication and continuity of care. This enabled these organizations to complement one another, providing a high quality service. To our knowledge this is the first controlled study that has investigated the effects of this kind of intervention on the quality of life of direct caregivers.

The study shows (Table 3) that this intervention compared with standard care contributed significantly to a better OQOLI of direct caregivers at T2 and T4. At T3 no significant positive influence of the intervention on the $O Q O L I$ of the direct caregiver was observed. This may seem inconsistent, but may be explained by the lower number of direct caregivers that could be analyzed for this time frame (possible Type II error).

When examining the mean score development of the 4 separate dimensions used to construct the OQOLI (Table 4), it may be speculated that the positive contribution of the intervention on the $O Q O L I$ at T2 may be owing to improvements on fear, loneliness, and general well-being scales, whereas at T4 this may be caused by improvements on the SIP and fear scales. These improvements were only observed in the intervention and not in the control group. However, as the number of patients in the control group is low, one has to be cautious in drawing definitive conclusions.

These findings of positive effects of the transmural home care program on the OQOLI of the direct caregivers of terminal cancer patients can be criticized on two fronts. First, a selection bias may have occurred due to drop-out. However, because drop-out in the intervention group was not significantly different from that in the control group and because direct caregivers who dropped-out matched the study group on initial characteristics, a selection bias occurring in this study seems unlikely. Moreover, dropout in the study group appeared to be fairly low (13.5\%) considering that a large number of drop-outs is not unusual such studies. ${ }^{4,36}$

The second criticism one can raise is whether the disadvantages of a quasi experimental design have been adequately met. Because the area in which the direct caregivers of the intervention group were residing is similar to that of the control group, especially regarding to the availability of home care services and the accessibility to the hospital, it is most unlikely that these different residential areas could explain our findings. Furthermore, a wide spectrum of initial patient characteristics and direct caregiver characteristics were collected. Eventually, the intervention group proved to be fairly comparable with the control group (Tables 1 and 2). Nevertheless, the data were extensively checked for potential confounders. Potential confounders together with the pre-intervention OQOLI (at T1) were added to the final multiple regression analyses. Because of these precautions it was concluded that the final results of this study are correct.

In conclusion, the transmural care intervention program, compared with standard 
community care, had a significantly positive effect on the $O Q O L I$ of direct caregivers of terminal cancer patients, both 1 week after discharge and 3 months after death of the patient. This finding can be explained by the enhanced coordination and cooperation between professional caregivers working in intramural and extramural care, leading to improved supportive care for these patients and their direct caregivers.

In view of these findings, as well as the observed positive contribution to the patient's quality of life with fewer re-admissions to hospital, ${ }^{37}$ health care policy makers should encourage further development of such transmural care programs. 


\section{References}

1. Fraumeni JF, Hoover RN, Devesa SS, Kinlen LJ. Epidemiology of cancer. In: Devita VT, Hellman S, Rosenberg SA, eds. Cancer. Principles \& practice of oncology. Philadelphia: J.B. Lippincott, 1993;196-236.

2. Steering Committee on Future Health Care Scenarios. Cancer in the Netherlands: scenarios on cancer 1985-2000. Dordrecht: Kluwer Academic Publishers, 1988.

3. Macdonald N. The interface between oncology and palliative medicine. In: Doyle D, Hanks GWC, MacDonald N, eds. Oxford textbook of palliative medicine. Oxford: Oxford University Press, 1997;11-7.

4. Ahmedzai S. Quality of life research in the European palliative care setting. In: Osaba D, ed. Effect of cancer on quality of life. London: CRC Press, 1991;323-32.

5. van den Akker PAM, Tits MHI, Kok NM. Leven met de dood. Over terminale patienten en terminale zorg in Nederland [Living with death. Terminal patients and terminal care in The Netherlands]. Houten: Bohn Stafleu Van Loghum, 1994.

6. Field D, James N. Where and how people die. In: Clark D, ed. The future of palliative care. Buckingham: Open University Press, 1993;6-29.

7. Wilkes E. Dying now. Lancet 1984;i:950-2.

8. Jones RV, Hansford J, Fiske J. Death from cancer at home: the carers' perspective. $B M J$ 1993;306:249-51.

9. O'Hare PA, Yost LS, McCorkle R. Strategies to improve continuity of care and decrease rehospitalization of cancer patients: a review. Cancer Invest 1993;11:140-58.

10. Conkling VK. Continuity of care issues for cancer patients and families. Cancer 1989;64:290-4.

11. Schrijvers AJP. Health and Health Care in the Netherlands. A critical Self-assessment by Dutch Experts in the Medical and health Sciences. Utrecht: De Tijdstroom B.V. 1997.

12. McCorkle RG, Benoliel JQ, Donaldson G, Georgiadou F, Moinpour C, Goodell B. A randomized clinical trial of home nursing care for lung cancer patients. Cancer 1989;64:1375-82.

13. Greer DS, Mor V, Morris JN, Sherwood S, Kidder D, Birnbaum H. An alternative in terminal care: results of the National Hospice Study. J Chronic Dis 1986;39:9-26.

14. Kane LK, Wales J, Bernstein L, Leibowitz A, Kaplan S. A randomised controlled trial of hospice care. Lancet 1984;i:890-4. 
15. Cummings JEB, Hughes SL, Weaver FM, Manheim LM, Conrad KJ, Nash $\mathrm{K}$, et al. Cost-effectiveness of Veterans Administration hospital-based home care. A randomized clinical trial. Arch Intern Med 1990;150:1274-80.

16. Hinton J. Can home care maintain an acceptable quality of life for patients with terminal cancer and their relatives? Palliat Med 1994;8:183-96.

17. McMillan SC, Mahon M. The impact of hospice services on the quality of life of primary caregivers. Oncol Nurs Forum 1994;21:1189-95.

18. Dawson NJ. Need satisfaction in terminal care settings. Soc Sci Med 1991;32:83-7.

19. Nationale Raad voor de Volksgezondheid/College voor Ziekenhuisvoorzieningen. Transmurale somatische zorg [Transmural somatic care]. Zoetermeer: Nationale Raad voor de Volksgezondheid, 1995.

20. Commissie modernisering curatieve zorg. Gedeelde zorg; betere zorg [Shared care; better care]. Zoetermeer: Commissie Modernisering Curatieve Zorg, 1994.

21. Cook TD, Campbell DT. Quasi-experimentation. Design \& analysis issues for field settings. Boston: Houghton Mifflin Company, 1979.

22. Polit DF, Hungler BP. Nursing research. Principles and methods. Philadelphia: J.B. Lippincott company, 1995.

23. Tabachnick BG, Fidell CS. Using multivariate statistics. New York: HarperCollins College Publishers, 1996.

24. de Witte LP. After the rehabilitation centre. A study into the course of functioning after discharge from rehabilitation [Thesis]. Amsterdam/Lisse: Swets \& Zeitlinger, 1991.

25. Scheurs PJG, Tellegen B, van de Willege G. Coping lijst [Health, stress and coping: The development of the Utrecht Coping Scale]. Gedrag 1984;12:101-17.

26. Furer JW, Konig-Zahn C, Tax B. Het meten van gezondheidstoestand. Deel I [The measurement of health state. Part 1]. Assen: Van Gorcum, 1995.

27. Bruin AF, Diederiks JPM, de Witte LP, Stevens FCJ, Philipsen H. The development of a short generic version of the Sickness Impact Profile. J Clin Epidemiol 1994;47:407-18.

28. de Witte L, Jacobs H, van der Horst F, Luttik A, Joosten J, Philipsen H. De waarde van de Sickness Impact Profile als maat voor het functioneren van patienten [The value of the Sickness Impact Profile as a measure for functioning of patients]. Gezondheid \& Samenleving 1987;8:120-7.

29. Greenwald HP. The specificity of quality of life measures among the seriously ill. Med Care 


\section{Chapter 5}

$1987 ; 25: 642-51$.

30. van der Ploeg HM. Validatie van de Zelf-Beoordelings-Vragenlijst [Validation of the Dutch version of the Spielberger State-Trait-Anxiety Inventory]. Ned Tijdschr Psychologie 1980;35:243-9.

31. de Jong-Gierveld J. Eenzaamheid: een meersporig onderzoek [Loneliness: a diverse investigation]. Deventer: Van Loghum Slaterus, 1984.

32. de Jong-Gierveld J, Tilburg T. Manual of the loneliness scale. Amsterdam: Dep. Social Research Methodology, Free University, 1990.

33. de Jong-Gierveld J, Kamphuis F. The development of a rash-type loneliness scale. Appl Psychol Measurement 1985;9:289-99.

34. Andrews FM, Witchey SB. Social indicators of well-being. Americans' perceptions of life quality. New York: Plenum Press, 1976.

35. de Haes JCJM. Kwaliteit van leven van kanker patienten [Quality of life of cancer patients]. Amsterdam/Lisse: Swets \& Zeitlinger, 1988.

36. Osaba $\mathrm{D}$. Lessons learned from measuring health related quality of life in oncology. $J$ Clin Oncol 1994;12:608-16.

37. Smeenk FWJM, de Witte LP, van Haastregt JCM, Schipper RM, Biezemans JPH, Crebolder HFJM. Transmural care. A new approach in the care for terminal cancer patients: Its effects on re-hospitalization and quality of life. Patient Educ Couns 1997; accepted. 


\section{Appendix}

Results of principal component analysis on the direct caregiver's quality of life outcome measures (in this analysis every time only 1 factor was extracted)

\begin{tabular}{lcccc}
\hline Time point & T1 & T2 & T3 & T4 \\
EVI\% of variance & $2.3 / 57.8$ & $2.3 / 57.1$ & $2.1 / 52.2$ & $2.7 / 66.4$ \\
\hline Outcome measure & \multicolumn{5}{c}{ Factor loadings } \\
\hline Loneliness & .62 & .81 & .51 & .67 \\
Fear & .84 & .86 & .85 & .90 \\
SIP & .82 & .54 & .67 & .84 \\
General well-being & .54 & .76 & .80 & .82 \\
\hline
\end{tabular}

T1-T3: see text, EV = Eigenvalue; SIP = Sickness Impact Profile. 


\section{CHAPTER}

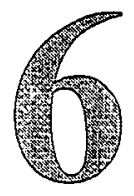

\section{Does a transmural home care intervention programme improve coordination and continuity of care?}

Submitted for publication as:

Smeenk FWJM, de Witte LP, Nooyen IWCJ, Crebolder HFJM. Does a transmural home care intervention programme improve coordination and continuity of care? 


\section{Abstract}

Although health care in The Netherlands is of a high quality with strong emphasis on primary care and high accessibility to hospital care, care deficits may arise in patients with chronic complex health problems who need the attention of several caregivers. Because no regular coordinating centre or person exists, coordination of care may be deficient leading to a poor continuity of care. This may be particularly true for those patients being cared for by both primary and hospital care teams, as is the case for most terminal cancer patients.

Therefore, a transmural home care intervention programme for terminal cancer patients was introduced which intended to optimize coordination of care and thereby improve continuity of care.

In a quasi-experimental study the effects of this intervention (including 79 patients) on indicators of coordination of care (i.e. task agreement among professional caregivers) and continuity of care (i.e. various aspects of communication and satisfaction with care) were compared to those of standard care (including 37 patients).

When considering the level of coordination of care, it was found to be poor. The various caregiver couples in the intervention group only showed a little more agreement on care tasks (3.9 out of 10 tasks) than those in the control group (3.7). Task agreement between the 'consultant specialist - specialist nurse coordinator' and 'hospital nurse community nurse' couples were significantly higher in the intervention group.

With regard to continuity of care, this study found that communication among caregivers in both groups was most adequate. This left little room for any improvement by the intervention on this indicator of continuity of care. Nevertheless, the consultant specialists were of the opinion that other caregivers in the intervention group were significantly better informed regarding the patient's discharge. Satisfaction with care was high for both patients, and their direct caregivers, in both groups (no significant differences between the groups).

In conclusion, this study showed slight positive effects on various indicators of coordination and continuity of care by the transmural home care intervention programme. Future prospective studies are needed to further elucidate the effects of transmural care on these aspects of care. 


\section{Introduction}

In The Netherlands, as in many other countries, health care is organized in 2 echelons. The first consists of primary (community) care, the second of hospital care. ${ }^{1}$ Health care in The Netherlands is characterized by its strong emphasis on primary care. Primary care consists of a general practitioner service available for consultation 24 hours a day, community nursing service (also available for 24 hours where necessary; i.e. 'intensive' community nursing), 'home help' for domestic assistance, 'meals on wheels', physiotherapy and a medical aids service which can provide equipment such as special beds, (wheel-) chairs etc. In the Dutch health care system the general practitioner functions as a 'gatekeeper' for other community and institutional services.'

For patients with acute uncomplicated health problems that can be solved by one caregiver, this system functions very well. For patients, however, with more problematic, complex and chronic health problems for which care is needed from several caregivers, this structure may show some deficits. ${ }^{2}$ No regular coordination of care facility is available which will bring together and integrate the care provided by these various caregivers. ${ }^{3}$ This may consequently lead to deficits in the continuity of care. Continuity of care is defined by most investigators as 'the degree to which professional and informal care is provided as a coordinated and uninterrupted sequence of activities, in accordance with the actual needs of the patient during the disease trajectory', ${ }^{2,4-6}$ Continuity of care can thus be seen as a dimension of the care process itself. The strategy which caregivers apply to enhance continuity of care, is regarded as the coordination of care..$^{3,47-9}$ Examples of coordination of care are: the introduction of a case manager/coordinator, the development of multidisciplinary care protocols, and the installation of regular team meetings.

In The Netherlands it has been shown that coordination of care was low (as measured by the degree of task agreement), especially between primary based and hospital care based caregivers, and that this lead to a considerable overlap in care., ${ }^{4,10}$ This problem may be of considerable importance to many patients as, in The Netherlands, it has been shown that as many as 9.3 percent of patients in a general practice will have chronic and complex health care problems. " Because terminal cancer patients are often cared for by various professional caregivers from both primary and hospital care teams, this coordination and continuity of care problem especially will affect them.

In order to improve the coordination and continuity of care, a transmural home care programme for these terminal cancer patients was introduced.

The main question of the present study was whether this transmural home care programme, focused on improving the coordination of care for terminal cancer patients, would show improvements in various aspects related to the coordination and continuity of care. 


\section{Methods}

\section{Design of the study (Figure 1)}

Due to ethical and practical reasons a randomized design was unfeasible. The main ethical reason was that the treating physicians considered it unethical to submit terminally ill patients to a randomization procedure. The main practical reason was the complexity of the health care settings. A randomized design would not only have required the cooperation of the many health care organizations within the city of Eindhoven (where the hospital was located and the intervention programme was based), but also that of those organizations in the surrounding urbanized areas. With a quasiexperimental design we could organize the intervention in Eindhoven initially and use the surrounding areas as a control group. Thus, patients being admitted to hospital and living in Eindhoven were allocated to the intervention group and those from the surrounding areas to the control group. After the patients' discharge from hospital (i.e. being the starting point of the intervention), patients from both groups received the standard care available in The Netherlands. Patients in the intervention group were offered the intervention programme additionally.

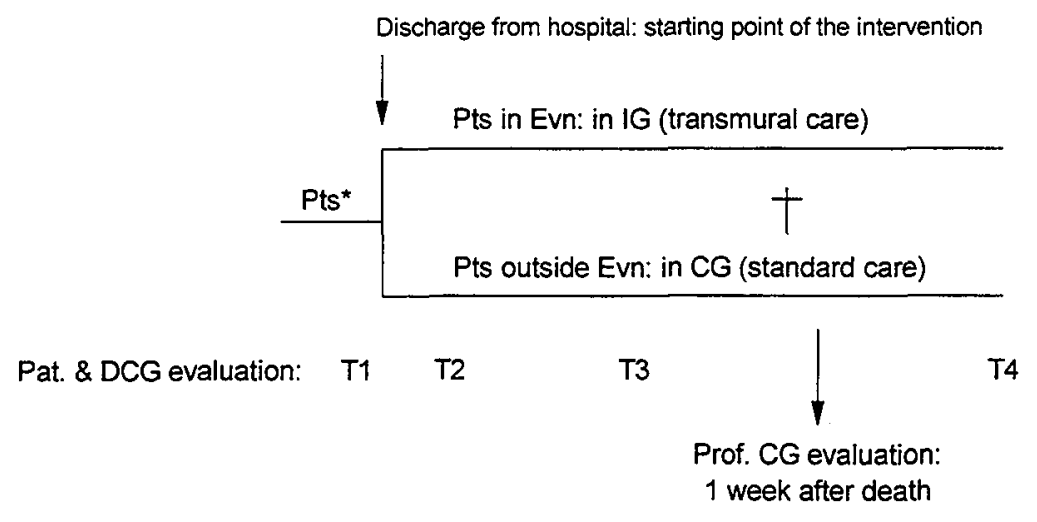

Figure 1 Study design

Prof. $\mathrm{CG}=$ professional caregiver; Pat. $=$ patient; $\mathrm{DCG}=$ direct caregiver; T1-T4; see text; $\mathrm{Pts}^{\star}=$ patients admitted to the hospital's multidisciplinary ward; Pts. $=$ patients; $\dagger=$ death of patient; Evn $=$ Eindhoven; $I G=$ intervention group; $C G=$ control group.

Indicators of coordination of care and continuity of care were evaluated by means of pre-structured questionnaires. Patients and their direct caregivers (that person who was considered to be the patient's principal informal caregiver i.e. being in most instances the patient's partner) received these questionnaires 1 week before (pre-intervention measurement: T1), 1 week after (T2), and 4 weeks after (T3) the patient's discharge from hospital. The direct caregivers of patients also received a questionnaire 3 months 
after the patient had died (T4). Professional caregivers received a questionnaire 1 week after the patient had died.

The study was approved by the hospital's Medical Ethical Committee. All patients gave their informed written consent.

\section{The intervention programme}

The intervention was specifically aimed at improving the coordination of care and consisted of four main items, each enhancing coordination in a specific way.

First, specific care protocols were set up by a multidisciplinary team. These protocols helped to standardize specific care and skills.

Second, a Specialist Nurse Coordinator was appointed. Her task was to coordinate the discharge of patients from hospital, and to organize the care needed at home, tailored to meet his individual needs.

Third, a 24-hour Consultation Telephone Service was established in the Catharina hospital (i.e. the hospital where the intervention was based). A member of the primary care team could use this service if and when problems arose. The service was manned by trained nurses from the oncology ward and the Accident \& Emergency Department. If the problem, often a technical nursing problem, could not be solved by the caregiver in the home setting, a hospital nurse from the consultation team could pay the patient a home visit (See Figure 2). If necessary, a medical specialist was available for consultation.

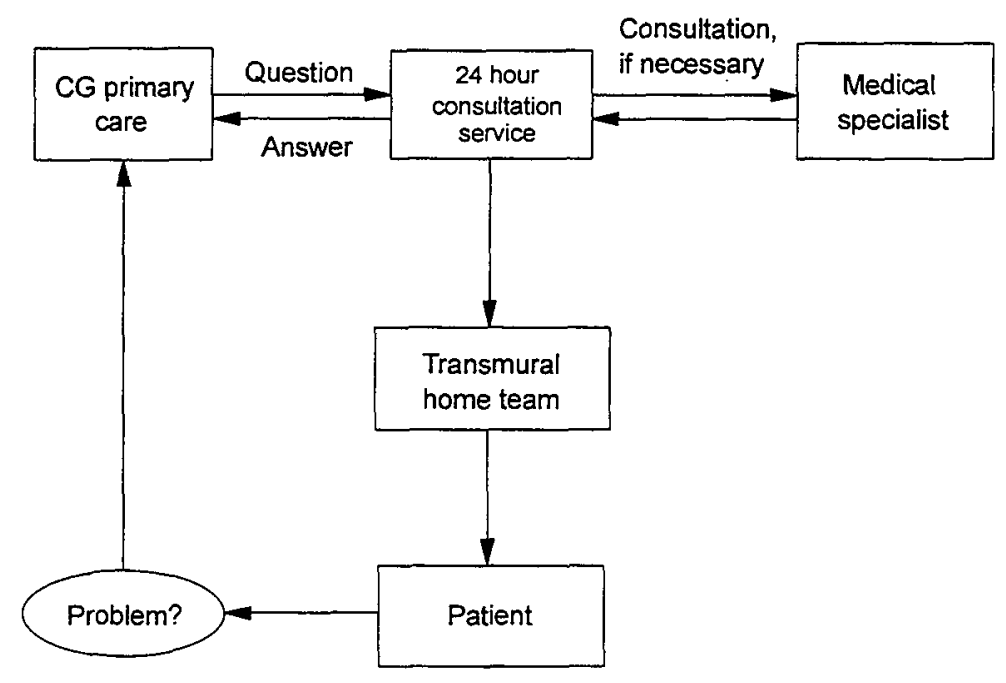

Figure 2 Organization structure of the 24-hour telephone consultation service and the transmural home team (CG = caregiver)

Fourth, a dossier kept at home with the patient was introduced. Each caregiver reported his/her findings and actions undertaken. It contained all the necessary medical and 


\section{Chapter 6}

nursing information.

\section{Patients}

Patients who were admitted to the hospital's multidisciplinary oncological unit and who met the following inclusion criteria: cancer, an estimated prognosis of less than 6 months, age 18 years or older, and being fully informed concerning diagnosis, could be included into the study. Patients were included from January 1994 till the first of February 1995.

Data concerning the patient's personal, socio-demographic and disease characteristics were collected on entry to the study. The complexity of their health problems at the start of the study (T1) were measured by counting the number of caregivers involved in the care of the patient at that moment.

\section{Outcome measures}

The levels of coordination of care and continuity of care were considered as outcome measures.

\section{Coordination of care}

The degree of agreement on care tasks among professional caregivers was considered as an indicator for the level of coordination of care. This was measured, in accordance with several previous studies, ${ }^{4,10}$ by asking caregivers 1 week after the patient's death which care tasks they thought belonged to which caregiver for that particular patient. The specific care tasks laid before the caregivers can be found in the appendix. These were adopted from a previous study by Courtens. ${ }^{4}$ When looking at the degree of task agreement amongst caregivers, pairs of caregivers (couples) were evaluated, specifically those who would have had the most intensive contacts during the care process (See Table 2).

\section{Continuity of care}

Communication and satisfaction with care are recognized by most investigators as important indicators of continuity of care. ${ }^{12-16}$

In the questionnaires addressing the various communication aspects, the following questions were put before the patients and their caregivers:

- At T2 the patients were asked how many days passed, after discharge from hospital, before caregivers visited them at home (i.e. this may be seen as reflecting the communication process between hospital and primary care regarding the patient's discharge).

- Patients (at T3) and their direct caregivers (at T4) were asked with which caregivers they had contact during their stay at home.

- Patients, their direct caregivers (at T2) and their professional caregivers (1 week after the patient died) were asked to what extent caregivers were informed about the discharge of the patient (rating on a 5-point scale from $1=$ poor till $5=$ most adequate) and whether they had problems in contacting caregivers. 
- Professional caregivers were asked, 1 week after the patient died, which caregivers they had contact with during and after the patient's discharge from hospital.

Satisfaction with care was evaluated by asking the professional caregivers:

- To judge the discharge arrangements after the patient's discharge (rating 'very poor' to 'very good'; 5-point scale).

- To give 'marks out of 10 ' ( 10 being the best possible rating) for the overall coordination of care.

The patients (at T3) and their direct caregivers (at T4) were asked to give 'marks out of 10 ' for the intervention as a whole.

\section{Analyses}

For all statistical analyses the SPSS package for windows (Statistical Package Social Sciences International BV, Gorinchem, The Netherlands) was used. Appropriate tests were used for comparing the variables according to their distribution. ${ }^{17} \mathrm{~A}$ p-value of .05 or less was considered significant.

\section{Results}

\section{Patients}

Seventy-nine patients could be included in the intervention and 37 in the control group. The intervention group and control group appeared to be fairly comparable with regards to initial patient characteristics (Table 1). The patients averaged 64 years of age and approximately $60 \%$ of them were men. The only difference found was that patients in the control group were more likely to still be living with their partner. There were therefore probably slightly more women and persons present in the household too.

At the start of the study ( $T 1$ ), the mean number of professional caregivers involved in the care of these patients was not significantly different in both groups. So the complexity of care at the start of the study was equal for both groups of patients.

The patients studied were a true representation of cancer patients in the 'terminal' phase. The mean survival of patients in the intervention group was 101.2 (standard deviation [SD] 141.5) days and 68.6 (SD 81.5) days in the control group (difference not significant). ${ }^{18}$

\section{Outcome measures \\ Coordination of care}

When considering the general opinion of the various professional caregiver categories regarding which care tasks they considered the responsibility of which specific caregiver category, the overall agreement on care tasks between the various caregiver categories was poor. Complete agreement was only found for care task no. 3 (i.e. 'the provision of medical information about the disease and options for treatment'), where almost all caregivers felt that this task should be carried out by the consultant specialist. Only 2 caregiver couples agreed on more than 5 tasks (i.e. the 'consultant specialist - general 
practitioner' couple agreed on 6 care tasks, and the 'specialist nurse coordinator hospital nurse' agreeing on 9 tasks).

Table 1 Initial patient characteristics

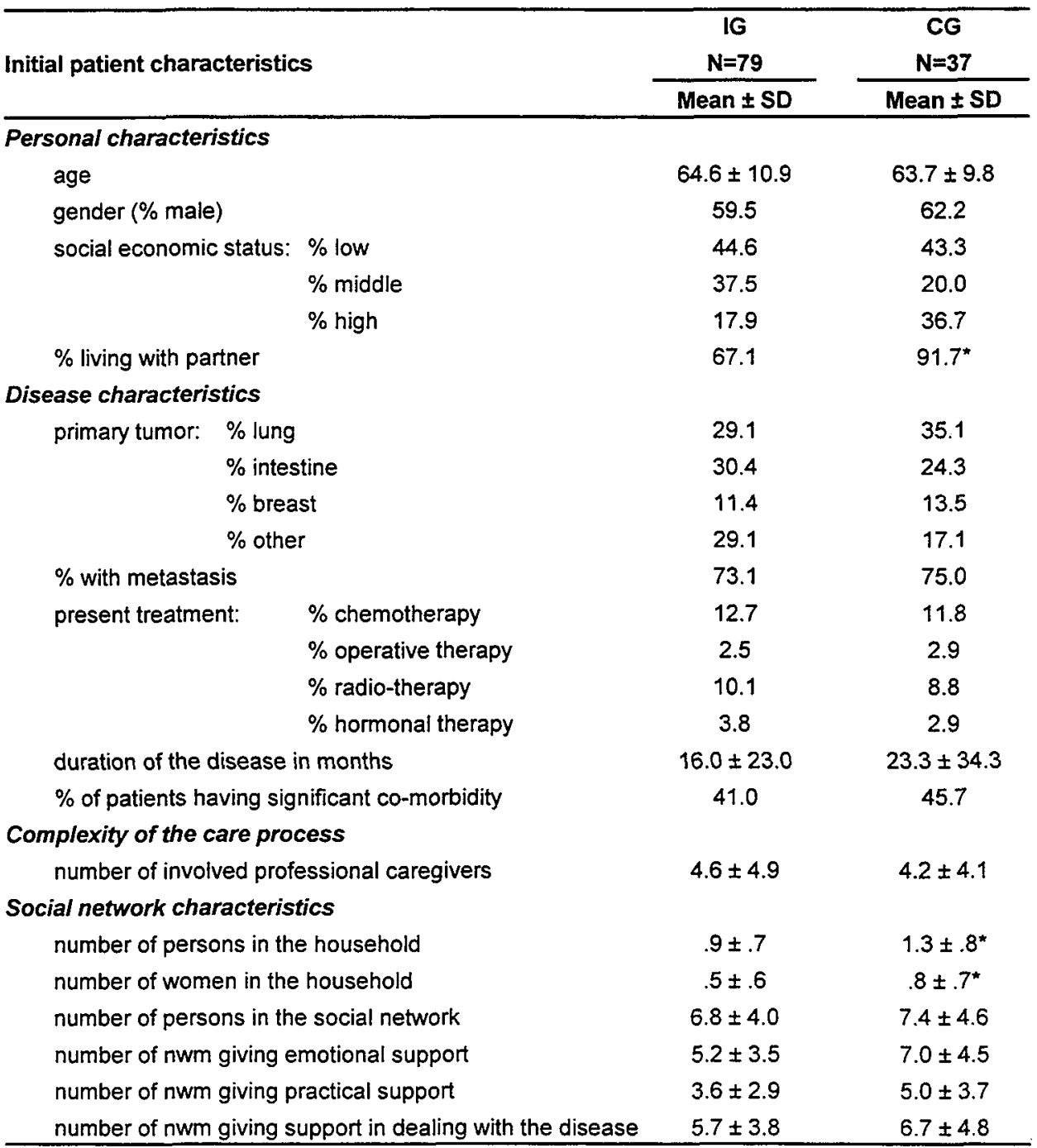

$1 G$ = intervention group, $C G=$ control group, $N=$ number of patients, $S D=$ standard deviation, $\mathrm{Nwm}=$ network members. No significant differences between the 2 groups were seen, except where otherwise indicated: ${ }^{\star}=p<.05$. For continuous and normally distributed variables the student's $t$ test were applied. For all other comparisons the $x^{2}$ test were applied. 
Table 2 Mean number of care tasks various caregiver couples agreed upon

\begin{tabular}{lcc}
\hline CG couple & $\begin{array}{c}\text { IG } \\
(\mathbf{N}=45-61)\end{array}$ & $\begin{array}{c}\text { CG } \\
(\mathbf{N}=\mathbf{1 7 - 2 5})\end{array}$ \\
\hline Spec-SNC & 3.7 & $3.1^{\star}$ \\
Spec-HN & 3.5 & 3.4 \\
Spec-GP & 4.0 & 4.4 \\
HN-SNC & 5.7 & 5.5 \\
HN-CN & 4.4 & $3.8^{\star}$ \\
GP-SNC & 2.6 & 2.4 \\
GP-CN & 2.5 & 2.7 \\
CN-SNC & 4.4 & 4.6 \\
\hline Mean for all & 3.9 & 3.7 \\
\hline
\end{tabular}

See table 1. IG = intervention group, $C G=$ control group, $C G=$ caregiver, $S p e c=$ consultant specialist, $\mathrm{SNC}=$ specialist nurse coordinator, $\mathrm{HN}=$ hospital nurse, $\mathrm{GP}=$ general practitioner, $\mathrm{CN}=$ community nurse, $\mathrm{HH}=$ home help, ${ }^{*}=$ difference significant (student's $t$ test; $p<.05$ )

The mean number of care tasks that individual professional caregiver couples agreed upon in the care of their individual patients are depicted in Table 2. This number averaged 3.9 in the intervention group and 3.7 in the control group (difference not significant). The 'consultant specialist - specialist nurse coordinator' couples in the intervention group agreed significantly more upon tasks than those in the control group (3.7 vs. 3.1). This was also true for the 'hospital nurse - community nurse' couple in the intervention group (4.4. vs. 3.8). The highest mean number was found for the 'hospital nurse - specialist nurse coordinator' couple in the intervention group (5.7); the lowest number was found for the 'general practitioner - specialist nurse' coordinator couple in the control group (2.4).

Where differing opinions exist on which care tasks should be performed by which caregiver, 2 consequences may result. First, both caregivers may view this task as belonging to themselves, in which case the task will be repeated by 2 caregivers. Second, 2 caregivers may feel that the task belongs to another, in which case the task will not be executed at all. The second possibility, both caregivers viewing the task the responsibility of another, appeared to be negligible. It was only observed in a minority of cases for task no. 4 (i.e. 3 out of 57 'consultant specialist - general practitioner' couples; 1 out of 63 'hospital nurse - community nurse' couples; and 3 out of 58 'consultant specialist - hospital nurse' couples), for task no. 8 (i.e. 5 out of 79 'community nurse - specialist nurse coordinator' couples), and for task no. 9 (i.e. 1 out of 72 'general practitioner - specialist nurse coordinator' couples; 1 out of 71 'specialist nurse coordinator - hospital nurse' couples; and 3 out of 65 'community nurse - specialist nurse 
coordinator' couples).

\section{Continuity of care}

With respect to the indicators for continuity of care, we found:

- Sixty five percent of the patients in the intervention and $71 \%$ of the patients in the control group were visited within the first 2 days after discharge by their general practitioner. These percentages were 72 and 64 respectively for the community nurse.

- With regard to the communication between patients and their (professional) caregivers (Table 3) at $\mathrm{T} 3$, significantly less patients in the intervention group reported having had contact with their consultant specialist, hospital nurse and general practitioner, whereas significantly more patients claimed to have had contact with their community nurse and home helper.

Table 3 Percentage of patients (at T3) and direct caregivers (at T4) reporting to have had contact with a particular professional caregiver

\begin{tabular}{lccccccccc}
\hline & Group & N & Spec & SNC & HN & GP & CN & HH & Mean \\
\hline Pat. T3 & IG & 35 & $29^{*}$ & $40^{*}$ & $17^{*}$ & $76^{*}$ & $77^{*}$ & $49^{*}$ & 2.9 \\
& CG & 10 & 80 & 0 & 30 & 90 & 60 & 40 & 3.0 \\
DCG T4 & IG & 57 & 98 & 86 & 96 & 100 & $89^{*}$ & $47^{*}$ & 5.2 \\
& CG & 22 & 100 & 86 & 100 & 100 & 95 & 27 & 5.1 \\
\hline
\end{tabular}

See table 2. T3 and T4: see text. Mean represents the mean number of caregivers the patient or direct caregiver had contact with, Pat $=$ patient, $D C G=$ direct caregiver, ${ }^{*}=$ difference significant (student's $t$ test; $p<.05$ )

With regard to the communication between direct caregivers and (professional) caregivers at $\mathrm{T} 4$, significantly more direct caregivers reported having had contact with their home helper, whereas slightly less said to have had contact with their community nurse.

Contacting the various caregivers when needed did not seem to be a problem at all for patients and their caregivers.

- In general, patients and their direct caregivers in both groups judged their various professional caregivers as being 'adequately' to 'most adequately' informed about the patient's discharge (mean rating $3.8-4.6$ on a 5 point scale at the different timepoints). The only significant difference found in this area was that the consultant specialists in the intervention group viewed their colleague caregivers somewhat better informed about patient discharge (mean 3.8: SD .2 on a 5-point scale) compared to the views of those consultant specialists in the control group (mean 2.2; SD $.1: \mathrm{p}<.05)$.

- With regard to the communication amongst professional caregivers, we failed to 
find obvious differences between the intervention and control group (Table 4). Only the community nurse in the intervention group had significantly less contact with other caregivers before the patient's discharge (See Table 4: 1.4 vs. $2.1 ; p<.05$ ).

Table 4 also shows that the outcome of the study may be influenced by a contamination bias i.e. the specialist nurse coordinator also reported having had contact with other caregivers for patients in the control group.

When considering the satisfaction with care analysis, this study found that the various caregivers' opinion on the patient's care transfer from hospital to primary care was on average high (mean of 3.9 on a 5 point scale in the intervention group and 4.0 in the control group).

The mean 'marks out of $10^{\prime}$ ' given by the various caregivers for the way care was coordinated ranged from 7.1 (home helpers in the intervention group; SD 1.4) to 8.1 (consultant specialists in the intervention and control group; SD .7).

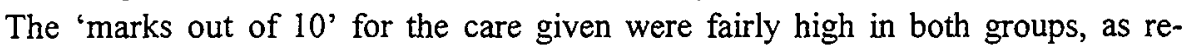
warded by patients and their direct caregivers. Patients in the intervention and the control group rated this 8.3 (SD .9) and 8.2 (SD 1.1) respectively. Direct caregivers in the intervention and control group rated this 8.6 (SD 1.0) and 9.1 (SD .9) respectively.

Table 4 The mean number of different caregivers with which a specific professional caregiver reported to have had contact with before - and after the patient's discharge

\begin{tabular}{|c|c|c|c|c|}
\hline \multirow[b]{3}{*}{ Caregiver } & \multicolumn{2}{|c|}{ Before discharge } & \multicolumn{2}{|c|}{ After discharge } \\
\hline & IG & CG & IG & CG \\
\hline & $\operatorname{mean}(\mathrm{n})$ & mean $(n)$ & mean $(n)$ & $\operatorname{mean}(\mathrm{n})$ \\
\hline Specialist & $2.3(61)$ & $2.4(25)$ & $.7(59)$ & $1.0(23)$ \\
\hline SNC & $3.5(75)$ & $2.6(28)^{\star}$ & $2.0(72)$ & $1.1(24)^{\star}$ \\
\hline$H N$ & $2.5(65)$ & $2.7(26)$ & $.6(48)$ & $.5(22)$ \\
\hline GP & $1.7(67)$ & $2.0(26)$ & $1.9(66)$ & $2.2(25)$ \\
\hline $\mathrm{CN}$ & $1.4(61)$ & $2.1(20)^{\star}$ & $1.9(61)$ & $1.8(22)$ \\
\hline $\mathrm{HH}$ & $1.1(19)$ & $.5(4)$ & $1.4(19)$ & $1.3(4)$ \\
\hline
\end{tabular}

See table 2. $n=$ total number of caregivers that could be analysed, ${ }^{*}=$ difference significant (student's $t$ test; $p<.05$ ).

With respect to these various outcome measures on satisfaction, no significant differences could be found between the intervention and control group.

\section{Discussion}

This study showed slightly positive effects of the transmural home care intervention 
programme on coordination of care (i.e. task agreement being higher for the 'consultant specialist - specialist nurse coordinator' couple and 'hospital nurse - community nurse' couple in the intervention group), and continuity of care (i.e. the consultant specialists in the intervention group were of the opinion that the other professional caregivers were better informed about the patient's discharge when compared to the opinion of those specialists in the control group).

When looking at these results in more detail, some observations merit a mention. First, overall task agreement amongst caregivers appeared to be poor. The various caregiver couples in the intervention group agreed on a mean of 3.9 care tasks (out of a set of 10 tasks) whereas this number was 3.7 in the control group. If the questionnaires had been randomly filled in by all, this mean number would have been 1.7. Task agreement by the majority of caregivers was only observed for 1 task, i.e. that of giving the patient medical information about his disease and options for treatment. Caregivers viewed this task as belonging to the patient's consultant specialist. This poor agreement on care tasks has also been observed by others. ${ }^{4.10}$ However, despite this poor agreement, hardly any care tasks were found on which caregiver couples disagreed in such a way that the task would not be executed at all (i.e. every caregiver thinking that another is responsible for this task).

Second, this study shows that communication between patients and caregivers and amongst professional caregivers was not a problem at all for either the intervention or control group during the care process. Furthermore, most patients were visited at home by their general practitioner and community nurse within 2 days of discharge from hospital (indicating that these primary caregivers were indeed informed of the patient's discharge by hospital caregivers), and most caregivers felt that their colleagues were adequately informed about the patient's discharge.

Third, it should be noted that significantly less patients in the intervention group reported having had contact with their consultant specialist, hospital nurse and general practitioner at $\mathrm{T} 3$ when compared to those in the control group, whereas a significantly higher percentage of these patients claimed to have had contact with their community nurse and home helper. This can be explained by the fact that, due to the intervention programme, patients in the intervention group underwent less re-hospitalization. ${ }^{19}$ Because of this, patients in the intervention group probably needed more help from their community nurse and home helper, with less need of care by their general practitioner (for arranging the hospital admissions), specialist and hospital nurse.

Furthermore, community nurses in the intervention group reported that, before the patient's discharge, they had contact with significantly less other caregivers when compared to the community nurse in the control group. This can be explained by the presence of a specialist nurse coordinator in the intervention group. It was the task of this functionary to coordinate the care for the patient. In doing so, it may have relieved the community nurse of the task of preparing the home environment for the patient's discharge, thereby reducing the need for her to have contact with other caregivers.

Lastly, satisfaction with care of patients and their direct caregivers was excellent in 
both groups (mean 'marks out of 10': 8.2-9.1).

Previous studies showed that the programme had positive effects on the re-hospitalization of patients as well as the patients' and their direct caregivers' quality of life, ${ }^{19,20}$ suggesting that the quality of care, and more specifically the coordination and continuity of care, offered to patients in the intervention group would be higher. Why then were only slight effects of the intervention on coordination and continuity of care observed in this study? Several explanations are possible.

First, various aspects of communication under consideration in this study appeared to have been adequately arranged already. Therefore, it would be hard for any intervention programme to gain improvements on these aspects of communication (ceiling effect).

Second, the applied outcome measures may have been too insensitive. In this study we used pre-structured questionnaires asking patients and caregivers to recall several aspects of communication and task agreement. This may have mitigated possible existing differences which may then become undetectable. Furthermore, professional caregivers, who received their questionnaires 1 week after the patient died (thus, after actual care process cessation), may well have given socially desirable answers thereby introducing an observation bias.

Third, it is important that communication has at least 2 aspects. First, there is the quantitative aspect. In general, it may be assumed that the more frequent caregivers communicate about the care a patient should receive, the better the continuity of care for that particular patient. Second, there is the qualitative aspect to communication. This aspect is even more important. Caregivers can communicate frequently with each other but if they fail to understand one another, or have differing opinions about the care process, this will jeopardize the continuity of care. Because only quantitative aspects of communication were looked at in this study, and because the intervention was specifically designed to improve the coordination of care, it seems reasonable to assume that the qualitative aspects of communication in particular explain the overall better outcome measures on re-hospitalization and patient's quality of life. ${ }^{19}$ However, this study did not evaluate this qualitative aspect, and therefore possible effects of the intervention on this area of communication would have gone unnoticed.

Fourth, due to ethical reasons it appeared impossible for us to withhold all the services of the intervention to all patients in the control group. As can be seen from Tables 3 and 4 the specialist nurse coordinator was also involved in the care of some patients in the control group. This would have minimized possible differences in the outcome measures between the 2 groups (contamination bias).

Fifth, patients and direct caregivers received their questionnaires during the care process. The direct caregivers also received a questionnaire 3 months after the patient's death. Because most of these patients (and their direct caregivers) required a great deal of medical and nursing attention, a 'please your doctor' bias (i.e. another observation bias) may have influenced their answers. This will probably have mitigated possible differences between the groups as well. 
Chapter 6

In summary, this study showed slightly positive effects of the transmural home care intervention programme on the indicators of coordination and continuity of care. Future prospective studies of a more qualitative nature will be needed to further elucidate the effects of transmural care on continuity and coordination of care. 


\section{References}

1. Schrijvers AJP. Health and Health Care in the Netherlands. A critical Self-assessment by Dutch Experts in the Medical and health Sciences. Utrecht: De Tijdstroom B.V. 1997.

2. Philipsen H, Stevens FCJ. Modernization, rationality, and continuity of care: Theoretical concepts and empirical findings. Sociological Focus 1997;30:189-204.

3. van Achterberg T, Stevens FCJ, Crebolder HFJM, de Witte LP. Coordination of care: effects on the continuity and quality of care. Int J Nurs Stud 1996;33:638-50.

4. Courtens AM. Kenmerken van zorg en kwaliteit van leven bij patienten met kanker ICharacteristics of care and quality of life in cancer patients; Thesis]. Maastricht: Universitaire Pers Maastricht, 1993.

5. Rogers J, Curtis P. The concept and measurement of continuity in primary care. Am J Public Health 1980;70:122-6.

6. van Achterberg T. Continuity of care and client satisfaction in the community. A study of professional and non-professional care for the chronically ill [Thesis]. Maastricht: Unigraphic, 1997.

7. Weiss LJ. Care coordination: An integration mechanism. In: Evanshwick CJ, Weiss LJ, eds. Managing the continuum of care. Rockville, MD: Aspen Publishers, 1987;271-91.

8. Austin $\mathrm{CD}, \mathrm{O}^{\prime}$ Connor $\mathrm{K}$. Case management: Components and program contexts. In: Petersen $\mathrm{MD}$, White DL, eds. Care of the elderly: An information source book. Newbury Park, CA: SAGE, 1989;167-205.

9. Alter C, Hage J. Organizations working together. Newbury Park, CA: SAGE, 1993.

10. de Witte LP. After the rehabilitation centre. A study into the course of functioning after discharge from rehabilitation [Thesis]. Amsterdam/Lisse: Swets \& Zeitlinger, 1991.

11. Knottnerus JA, Metsemakers J, Hoppener P, Limonard C. Chronic Illness in the community and the concept of 'social prevalence'. Fam Pract 1992;9:15-21.

12. Hampson JP, Roberts RI, Morgan DA. Shared care: a review of the literature. Fam Pract 1996;13:264-79.

13. O'Hare PA, Yost LS, McCorkle R. Strategies to improve continuity of care and decrease rehospitalization of cancer patients: a review. Cancer Invest 1993;11:140-58.

14. Haines A, Booroff A. Terminal care at home: perspective from general practice. $\mathrm{Br} M e d J$ (Clin Res Ed) 1986;292:1051-3. 
Chapter 6

15. Cartwright $A$. The relationship between general practitioners, hospital consultants and community nurses when caring for people in the last year of their lives. Fam Pract $1991 ; 8: 350-5$.

16. Hjortdahl $P$, Laerum E. Continuity of care in general practice: effect on patient satisfaction. $B M J$ 1992;304:1287-90.

17. Armitage P, Berry G. Statistical methods in clinical research. Oxford: Blackwell scientific publications, 1987.

18. Wachtel T, Allen-Masterson S, Reuben D, Goldberg R, Mor V. The end stage cancer patient: terminal common pathway. Hosp $J$ 1988;4:43-80.

19. Smeenk FWJM, de Witte LP, van Haastregt JCM, Schipper RM, Biezemans JPH, Crebolder HFJM. Transmural care. A new approach in the care for terminal cancer patients: Its effects on re-hospitalization and quality of life. Patient Educ Couns 1997; accepted.

20. Smeenk FWJM, de Witte LP, van Haastregt JCM, Schipper RM, Biezemans JPH, Crebolder HFJM. Transmural care of terminal cancer patients: Effects on the quality of life of direct caregivers. Nurs Res 1997; accepted. 


\section{Appendix}

Care tasks laid before caregivers asking them which tasks they judged to be the primary concern of which caregivers.

Care task:

1. The supervision/guidance of patients during their stay in hospital.

2. The supervision/guidance of the patients' family during their stay in hospital.

3. The provision of medical information about the diseases and options for treatment.

4. The provision of health education and explanation about lifestyle.

5. Giving support in dealing with and accepting the disease.

6. Giving support to families with respect to possible problems due to the patient's illness.

7. Preparing the patient's discharge from hospital and his/her coming home.

8. The coordination of care after the patient's discharge.

9. Safeguarding the whole care process and, if necessary, taking the initiative to solve possible gaps in care provision.

10. To guide and inform patients on important settlements and institutions that are important for him/her. 


\section{CHAPTER}

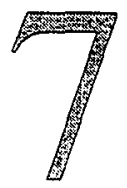

\section{A cost analysis of transmural home care for terminal cancer patients}

Accepted for publication as:

Smeenk FWJM, Ament AJHA, van Haastregt JCM, de Witte LP, Crebolder HFJM. A cost analysis of transmural home care for terminal cancer patients. Patient Educ Couns 1997. 


\begin{abstract}
The economic implications of home care service programmes for oncology patients remain unclear. This quasi-experimental study investigated the costs of a transmural home care programme for terminal cancer patients and compared them to those of the standard care available. The programme intended to optimize communication, cooperation and coordination between the intra- and extra-mural health care organizations (transmural care). Complete sets of data could be retrieved for 57 of the 79 patients in the intervention group, and for 29 of the 37 patients in the control group. Comparison of the intervention and control group revealed significantly lower pharmaceutical and rehospitalization costs in the intervention group, whilst community and home help costs were significantly higher. However, no significant difference could be found for total health care costs between both groups. In view of this, and that the programme has proved to have significantly positive effects on both the patient's and direct caregiver's quality of life, the installation of such programmes in every hospital with a multidisciplinary oncology unit is recommended.
\end{abstract}




\section{Introduction}

Despite all the technological innovations in treatment, cancer remains incurable in more than 50 percent of all cases.' Inevitably, most incurable patients will enter a terminal phase of their disease. This phase appears to be associated with high costs for both health care insurance companies and society at large. ${ }^{2,3}$ The most important reason for this is the high rate of re-hospitalization. ${ }^{4-6}$ The main causes of re-hospitalization are: inability to relieve symptoms at home, overburdened direct caregivers and inadequate communication between professional caregivers..$^{711}$

Home care programmes based at hospitals, hospices or the primary care setting have tried to reduce re-hospitalization rates and thereby health care costs as well. However, investigation results have, until now, been inconsistent. ${ }^{12-18}$ This may have several explanations, for instance: selection bias of those patients using these services, different assessment time frames, limited range of costs being evaluated et cetera. ${ }^{2.19}$ Furthermore, most studies can be criticized because they only considered costs refunded by insurance companies as their main outcome measure, which does not necessarily represent the real costs. $^{2}$

In order to be able to provide better supportive care for these patients at home, an intervention programme was developed. Its main objective was to offer the patient care specially tailored to meet his individual needs, and provided by professional caregivers from primary and hospital teams. In the intervention group the primary care team continued to care for the patient as usual, but with collaborative support from the hospital care team. The intervention intended to optimize care by improving the continuity of care and emphasizing the 'complementary approach' in care giving, with maximal coordination and communication. This kind of care was named transmural care in The Netherlands. ${ }^{20}$ The development of such care at a regional level was highly recommended by the Dutch government. ${ }^{21}$

Earlier reports showed that this transmural home care intervention programme, when compared to standard care, had a significantly positive effect on the patients' and their direct caregivers' quality of life, whilst simultaneously reducing re-hospitalization. $^{22,23}$

This study examined the cost aspects of this programme and compared them to that of the standard care available.

\section{Methods}

\section{Design of the study}

Due to ethical and practical reasons, the study design had to be quasi-experimental. These reasons are extensively discussed in an earlier paper reporting the effects of this transmural intervention programme on re-hospitalization and the patients' quality of life. ${ }^{22}$ Patients living in Eindhoven were allocated to the intervention group and those 
from the surrounding areas to the control group. Both groups received the standard care available in The Netherlands. ${ }^{24}$ In addition to this care patients included in the intervention group were also offered the intervention programme.

Ideally, an economic analysis should be performed from a societal perspective. ${ }^{2 s}$ However, the patients studied were all terminally ill and it could be safely assumed that possible productivity loss would be on the same low level for both groups. Consequently the analysis was confined to the health care perspective, i.e. the total sum of health care costs. The total costs were examined from inclusion until death.

When considering the design of the study it should be recognized that these 2 study areas are very similar. Eindhoven is a city with 200,000 inhabitants and a highly urbanized surrounding area. About 200,000 people also live in these urbanized surroundings. The maximum distance needed to travel to our hospital is about 30 kilometres (20 miles). This makes access to hospital very easy for all patients in the study. These areas are in fact so much urbanized that together they form 1 big city. The Dutch government has therefore recently decided to merge Eindhoven and its surrounding municipalities into one city province. Furthermore, the availability of home care services was similar in both groups and a pilot study using the data base of the Comprehensive Cancer Centre South did not reveal any major differences in the incidence of the various types of cancer, age, or gender of the cancer patients in these 2 populations.

The study was approved by the hospital's Medical Ethical Committee. All patients gave their written informed consent.

\section{The standard care available}

In The Netherlands health care is characterized by its strong emphasis on community care. ${ }^{24}$ Primary care consists of a general practitioner which service is available for 24 hours a day, community nurses (also available for 24 hours where necessary; i.e. 'intensive' [nocturnal] community nursing), 'home help' for domestic assistance, 'meals on wheels', physiotherapy and a medical aids service which can provide equipments such as special beds, (wheel-)chairs etc. The general practitioner functions as a 'gatekeeper' for other community and institutional services. The Dutch community and hospital health care services are highly accessible. Primarily because these services are fully covered by compulsory national or private insurance, or require only limited contributions. Furthermore, most people live within a relatively short distance from a hospital because of the high population density (average density of 400 per square kilometre; range $100-1,000$ ) in The Netherlands.

\section{The intervention programme}

The transmural home care intervention programme was specifically aimed at assisting the primary care team, and consisted of four main items: a specialist nurse coordinator, a 24-hour telephone service in the hospital with access to a transmural home team, a collaborative home care dossier (case file), and protocols designed for specific care. 


\section{The specialist nurse coordinator}

On request of the treating specialist, this functionary makes the necessary patient discharge arrangements. She monitors the care provision process, as well as tracking down and solving any possible defaults or shortcomings. The discharge of patients from the control group was planned, as usual, by the nurse responsible for his/her care.

The 24-hour telephone service and transmural home team (Figure 1)

A 24-hour telephone consultation service was installed on the hospital's multidisciplinary oncology ward. In case of problems arising at home this service could be contacted for advice, thus guaranteeing continuity of the care. If specific nursing problems could not be solved by the primary care team, the patient's general practitioner could request the support of trained nurses from the hospital's transmural home team.

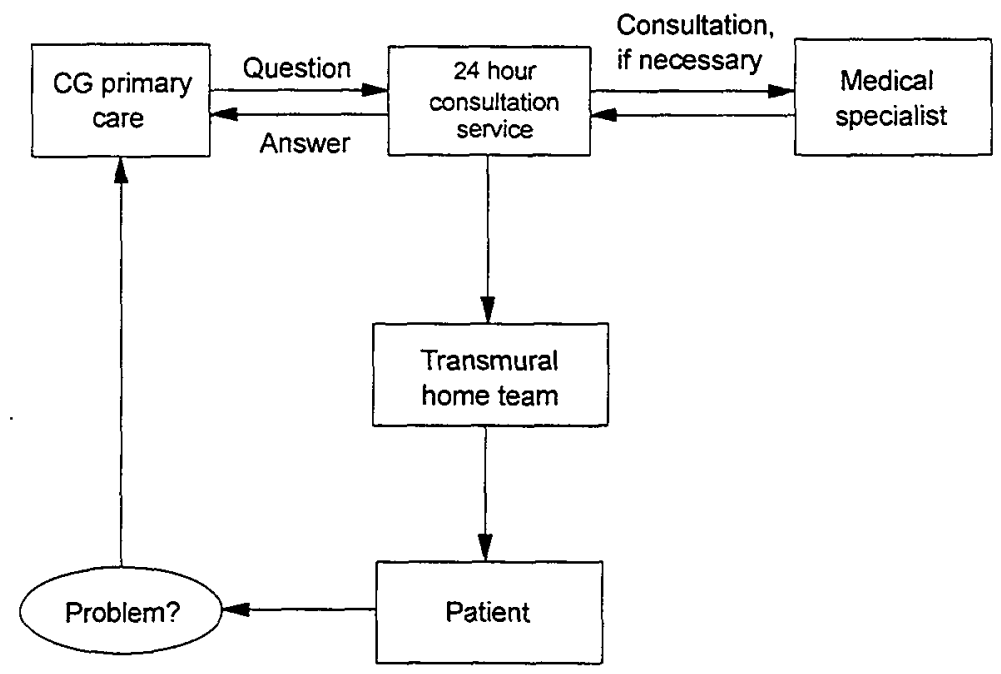

Figure 1 Organization structure of the 24-hour consultation service and the Transmural Home Team ( $\mathrm{CG}=$ caregiver)

The home care dossier

Caregivers from primary - and hospital care teams were asked to collaborate in reporting their findings, and actions undertaken, in this dossier. Patients themselves act as the actual trustees of their dossier. This ensures the continued availability of information on findings and care plans for all caregivers.

The care protocols

These were developed by a multidisciplinary team. Protocols that were introduced are: intravenous therapy, epidural-spinal pain relief, and the pharmaceutical trajectory.

A more detailed description of the intervention can be found elsewhere. ${ }^{22}$ It is 
important to stress that the implementation of this programme did not alter the existing health care organizational structure. So, the responsibility for patient admission to hospital remained that of the consultant specialist, and for patient care at home that of the general practitioner.

\section{Patients}

Those patients admitted to the hospital's multidisciplinary oncology ward and who matched the following inclusion criteria: cancer, an estimated prognosis of less than 6 months, age 18 years or older, and being fully informed of their diagnosis could be included in the study. Patients were included from January 1994 till the first of February 1995. Data concerning the disease, its treatment, the social network and social support, ${ }^{26}$ socio-demographic and personal characteristics were collected upon inclusion in the study.

\section{Outcome variables}

Total health care costs were divided into 3 categories:

1. Costs that were covered by health care insurance companies. Here, 6 sub-categories were distinguished: physician's fees, hospital admissions, outpatient/day care treatments, physiotherapy, drugs, transportation and medical aids supplied at home.

2. Costs made by home care facilities. In this category 3 sub-categories were distinguished: 'intensive' (nocturnal) community nursing care, standard community nursing care and (professional) home help.

3. Costs attributable to the intervention programme itself. In this category 3 sub-categories were distinguished: the specialist nurse coordinator, the 24-hour consultation telephone service and the Transmural Home Team.

Costs covered by insurance companies and for 'intensive' (nocturnal) community nursing care (in 1994 these costs were refunded by the insurance companies) could be retrieved from the databases of the two local insurance companies (VGZ and CZ), who cooperated with this study.

Standard community nursing care, home help costs and the costs for the intervention programme itself were recorded for the intervention group prospectively. For the control group these costs could only be retrospectively retrieved from the various home care agencies.

In accordance with the guidelines by Drummond et al., ${ }^{25}$ real costs made by these patients were considered wherever possible rather than tariffs. The average real costs of hospital admission per day for this patient category was calculated. Furthermore, the hourly rate of pay of the various caregivers were increased taking into account their 'unproductive' working hours and use of the various 'overheads'. For the calculation method see appendix. Costs refunded by insurance companies for physician's fees, hospital outpatient treatments, drugs and transportation and medical aid supplies were considered to represent the approximated real costs. 


\section{Analyses}

For all statistical analyses the SPSS package for windows (Statistical Package Social Sciences International B.V., Gorinchem, The Netherlands) was used. Appropriate tests were used for comparing the variables according to their distribution. ${ }^{27} \mathrm{~A}$ p-value of .05 or less was considered significant, except for the analyses performed for detecting possible confounders. Here, a conservative significance level of $\mathrm{p} \leq .10$ was used. To check for selection bias the initial patient characteristics of the final study group were compared to those of the 'drop-outs'. The intervention and control group underwent the same procedure. The costs made in the various categories were then compared between the intervention and control group.

Finally, a search was performed for potential confounders in the relationship between the experimental condition and the main outcome variables i.e. the total health care costs. Initial patient characteristics that were significantly related to this main outcome variable and which also showed a significant difference between the intervention and control group, were considered potential confounders. ${ }^{28}$

\section{Results}

\section{Patients}

Seventy-nine patients were included in the intervention group and 37 in the control group. In 86 of these patients complete sets of data on the various costs categories could be retrieved. The main reason for incomplete sets of data was that 25 cases were not insured by one of the 2 local insurance companies that cooperated with this study. In 3 cases the data on standard community nursing and home help were lost, as was the data for home help in 2 cases.

\section{Patients with incomplete data sets ('drop-outs')}

The percentage of incomplete sets of data in the intervention group (27.8\%) was not significantly different to that in the control group (21.6\%).

Comparing the initial patient characteristics of these 'drop-out' patients with the patients that could be evaluated, the only differences found were that significantly more patients in the 'drop-out' group had a high socio economic status (50.0 vs. $16.7 \%$; $\chi^{2}$ test, $\mathrm{p}=.01$ ) and patients in the 'drop-out' group had slightly less children living nearby ( 1.0 vs. 1.4 ; student's $t$ test, $\mathrm{p}=.05$ ).

\section{Initial patient characteristics}

The initial patient characteristics, displayed in Table 1, reveal that the intervention and control group were comparable. The control group did show significantly more patients with a partner at home and more household members. 
Table 1 The initial patient characteristics

\begin{tabular}{|c|c|c|c|}
\hline \multicolumn{2}{|c|}{ Initial patient characteristics } & IG $(N=57)$ & CG $(\mathrm{N}=29)$ \\
\hline \multicolumn{4}{|c|}{ Personal characteristics } \\
\hline \multicolumn{2}{|c|}{ age (mean age $\pm S D$ ) } & $63.4 \pm 10.4$ & $63.6 \pm 10.5$ \\
\hline \multicolumn{2}{|c|}{ gender ( $\%$ male $)$} & $56.1 \%$ & $62.1 \%$ \\
\hline \multirow[t]{3}{*}{ socio-economic s } & low & $43.9 \%$ & $52.0 \%$ \\
\hline & middle & $43.9 \%$ & $24.0 \%$ \\
\hline & high & $12.2 \%$ & $24.0 \%$ \\
\hline \multicolumn{2}{|l|}{ private insurance } & $8.8 \%$ & $6.9 \%$ \\
\hline \multicolumn{2}{|l|}{ living with partner } & $69.1 \%$ & $96.6 \%^{*}$ \\
\hline \multicolumn{4}{|c|}{ Disease characteristics } \\
\hline \multirow[t]{4}{*}{ primary tumor: } & & $29.8 \%$ & $37.9 \%$ \\
\hline & & $33.3 \%$ & $20.7 \%$ \\
\hline & & $15.8 \%$ & $13.8 \%$ \\
\hline & & $21.1 \%$ & $27.6 \%$ \\
\hline \multicolumn{2}{|l|}{ metastasis } & $76.8 \%$ & $75.0 \%$ \\
\hline \multirow[t]{4}{*}{ present treatment } & chemotherapy & $12.3 \%$ & $7.4 \%$ \\
\hline & operative therapy & $1.8 \%$ & $3.7 \%$ \\
\hline & radio-therapy & $10.5 \%$ & $3.7 \%$ \\
\hline & hormonal therapy & $5.3 \%$ & $.0 \%$ \\
\hline \multicolumn{2}{|c|}{ duration of the disease in months (mean $\pm S D$ ) } & $17.1 \pm 25.3$ & $21.8 \pm 35.0$ \\
\hline \multicolumn{2}{|c|}{ patients having significant co-morbidity } & $48.4 \%$ & $48.1 \%$ \\
\hline \multicolumn{2}{|c|}{ Social network characteristics } & Mean $\pm S D$ & Mean $\pm S D$ \\
\hline \multicolumn{2}{|c|}{ number of persons in the household } & $.9 \pm .6$ & $1.3 \pm .8^{*}$ \\
\hline \multicolumn{2}{|c|}{ number of persons in the social network } & $6.9 \pm 4.2$ & $7.5 \pm 4.7$ \\
\hline \multicolumn{2}{|c|}{ total number of contacts with nwm per year } & $98.4 \pm 64.1$ & $116.4 \pm 69.1$ \\
\hline
\end{tabular}

$I G=$ intervention group, $C G=$ control group, $N=$ number of patients, $S D=$ standard deviation, $\mathrm{Nwm}=$ network members. No initial patient characteristics were significantly different for both groups except where otherwise indicated: ${ }^{*}=p<.05$. The student's $t$ test was used for those continuous variables which showed a normal distribution. For all other comparisons the $x^{2}$ test was used.

\section{Outcome measures}

Table 2 shows that patients in the intervention group underwent significantly less rehospitalization (on average 5.3 vs. 12.7 days for their remaining life) and that the survival period of patients was not significantly different.

The average time personnel needed for patient care in the intervention programme is depicted in Table 3.

Table 4 displays the results of various cost category comparisons between the intervention and control group. It shows that the total costs per patient for drugs was significantly lower in the intervention group compared to the control group (579 vs. 957 
Dutch guilders [Dfl.]; in 1994 one US Dollar approximated 1.80 Dfl.). Also for hospital admissions (2,593 vs. 6,252 Dfl.). Costs for standard community nursing (1,351 vs. 206 Dfl.) and home help $(3,911$ vs. $770 \mathrm{Dfl}$.), however, were significantly higher in the intervention group. The total cost per patient for the intervention programme was 598 Dfl. The total sum of health care costs per patient in the intervention group $(14,287 \mathrm{Dfl}$.) was not significantly different from that in the control group (13,052 Dfl.).

Table 2 Re-hospitalization and survival

\begin{tabular}{lccc}
\hline & IG $(n=57)$ & & CG $(n=29)$ \\
\cline { 2 - 2 } & Mean \pm SD & & Mean $\pm S D$ \\
\hline Days in hospital & $5.3 \pm 12.1$ & & $12.7 \pm 16.7^{*}$ \\
Survival (in days) & $85.2 \pm 114.9$ & & $68.1 \pm 79.9 n s$ \\
Days in hospital/survival $\times 100$ & $6.5 \pm 14.0$ & & $24.0 \pm 25.2 \dagger$ \\
\hline
\end{tabular}

$I G=$ intervention group, $C G=$ control group, $S D=$ standard deviation, $n=$ number of patients. For all statistical comparisons the Mann-Whitney $U$ test was used; ns = not significant, ${ }^{*}=p<.01, t=p<.001$.

Table 3 Average time investment (in minutes) \pm SD per patient by the intervention programme personnel

\begin{tabular}{lcc}
\hline & $\begin{array}{c}\text { Total intervention group } \\
(n=79)\end{array}$ & $\begin{array}{c}\text { Study group* } \\
(n=57)\end{array}$ \\
\hline Specialist nurse coordinator & $453 \pm 246$ & $420 \pm 203$ \\
24 Hour telephone service & $12 \pm 23 \dagger$ & $15 \pm 25 \|$ \\
Transmural home team & $8 \pm 28 \S$ & $6 \pm 23 \pi$ \\
\hline
\end{tabular}

* = those patients from the intervention group with complete sets of data on the various cost categories, $\dagger=$ this service was contacted 100 times in total during the study period , $\S=$ the out-reach team had to visit patients on 8 occasions in total, $\|=$ this service was contacted 80 times in total,$\pi=$ the out-reach team had to visit the patient at home on 5 occasions in total.

The search for potential confounders revealed 2 possible confounders: marital status and the number of persons in the household. These 2 confounders highly correlated with one another $(\mathrm{R}=.60 ; \mathrm{p}<.001)$. In order to correct for these possible confounders, a hierarchical backward multiple regression analysis was performed. The 2 possible confounders were added to the experimental condition as independent variables in the regression analysis on total health care costs. This analysis confirmed our previous findings that the intervention did not significantly influence the total health care costs.

In addition to these calculations, the same analysis was performed using the health 
Chapter 7

care tariffs instead of real costs. This analysis resulted in the same conclusions.

Table 4 Average health care costs (in Dutch guilders) \pm SD per patient

\begin{tabular}{lcc}
\hline & $\begin{array}{c}\text { Intervention Group } \\
(\mathbf{n}=57)\end{array}$ & $\begin{array}{c}\text { Control Group } \\
(\mathbf{n}=29)\end{array}$ \\
\hline Costs made by the insurance companies & & \\
Physician's aid & $1445 \pm 2847$ & $1489 \pm 2511$ \\
Drugs & $579 \pm 1309$ & $957 \pm 1442^{*}$ \\
Physiotherapy & $42 \pm 146$ & $14 \pm 75$ \\
Hospital admission & $2593 \pm 5958$ & $6252 \pm 8222 \dagger$ \\
Day treatment in hospital & $59 \pm 198$ & $26 \pm 97$ \\
Transportation and medical aid supplies & $1350 \pm 2436$ & $1117 \pm 1039$ \\
\hline Total costs insurance companies & $6069 \pm 8778$ & $9854 \pm 10137 \dagger$ \\
\hline Costs for home nursing & & $1810 \pm 4737$ \\
"Intensive" home nursing & $2621 \pm 5818$ & $206 \pm 663 \S$ \\
Home nursing & $1351 \pm 2427$ & $770 \pm 2067 \dagger$ \\
\hline Family aid & $3911 \pm 7581$ & $2295 \pm 5263 \S$ \\
\hline Total costs for home nursing & $7930 \pm 12397$ & - \\
\hline Costs for the intervention programme & & - \\
Specialist Nurse Coordinator & $571 \pm 276$ & - \\
24 Hour Consultation Service & $16 \pm 27$ & - \\
Home Care Team & $10 \pm 36$ & $13052 \pm 11477$ \\
\hline Total costs of the intervention & $598 \pm 283$ & \\
\hline \hline TOTAL CosTS & $14287 \pm 15285$ & \\
\hline
\end{tabular}

$\mathrm{n}=$ number of patients, differences not significant except where otherwise indicated: * $p \leq .05, \dagger=p \leq .01, \S=p \leq .001$. Comparisons were performed using the Mann-Whitney $U$ test.

Because some costs for community nursing in the control group may have been lost, due to retrospective data gathering in this group, the observed differences in costs for community nursing and home help (costs for home help in the control group had to be estimated using the cost's ratio between the intervention and control group for community nursing and the cost's ratio: See Appendix) between the two groups may represent an overestimation. This becomes more probable when one takes into account the fact that the agencies supplying home care for the control group changed their data file system during this period. The approximate 6-fold rise in costs for these items seems even more unlikely when one considers the following: If patients in the intervention group did indeed have a greater need for community nursing and home help, one may 
reasonably expect a similar increase in 'intensive' community nursing care costs. This, however, was not the case. As data on 'intensive' community nursing care were retrieved similarly for both groups (provided by the insurance companies because these costs were refunded by them in 1994), we feel that this probably gives a more realistic view of the real differences in home care needs between the patients in the intervention and control group. Therefore, an alternative cost comparison was made by assuming that the costs ratios between the intervention and control group for both community nursing and home help would be the same as the costs ratio for 'intensive' community nursing between these groups. With the knowledge of this last ratio, plus the costs for community nursing and home help in the intervention group, community nursing and home help costs for the control group could be calculated. If these assumptions are valid, the average costs per patient for community nursing and home help in the control group were $933 \mathrm{Dfl}$. and 2,701 Dfl respectively. In this scenario, the average total costs per patient in the control group was calculated as being $15,710 \mathrm{Dfl}$.

\section{Discussion}

This study shows that our transmural care programme significantly reduced costs for drugs and re-hospitalization. The costs for standard community nursing care and home help were significantly higher in the intervention group. However, the total sum of health care costs was not significantly different between both groups. Several limitations of this study are discussed below.

First of all, 'drop-out' may have biased our results. Comparing the 'drop-out' patients (those patients with incomplete sets of data concerning their cost analysis) with the patients who could be evaluated, differences were only found in socio economic status and the number of children living nearby amongst a broad set of initial patient characteristics. Furthermore, 'drop-out' in the intervention group was not significantly different from that in the control group. Therefore, it seems unlikely that selection due to 'drop-out' would have influenced our results.

Secondly, the quasi-experimental design of the study may have influenced our final results. When comparing the intervention group with the control group on their initial patient characteristics which covered a broad spectrum of disease, personal and social network characteristics, the 2 groups appeared to be comparable. Moreover, these results were checked for confounders. Only 2 were found: marital status and the number of persons in the household. These correlated highly with each another, as could be expected. Patients in the control group were more likely to have a partner at home (who also acted as the direct caregiver) and to have more persons in the household. It may therefore be assumed that if patients in the control group had had a family/social network similar to that of the intervention group, community nursing and home help costs would also have been higher than we observed. The differences observed in costs for standard community nursing and home help may then be an overestimation of the real effect. On the other hand, the difference observed in costs for hospital admissions may 
be an underestimation of the real effect. Patients living in 'weaker' family/social networks are more likely to be re-admitted to hospital. ${ }^{29-31}$ So, it is extremely unlikely that these confounders will have influenced our final result: i.e. that the intervention programme did certainly not result in higher health care costs, compared to standard care. This was confirmed by a multiple regression analysis in which these confounders were added as independent variables.

Neither is the difference in residential areas between the intervention and control group likely to have influenced the final result because the 2 areas are similar (they will even be merged into one city province in the near future), especially with respect to hospital accessibility (similar short travelling distances to hospital) and the availability of home care services. Therefore, it is also unlikely that this would have biased our results. If one, nevertheless, still feels that this possible bias cannot be ruled out all together, one should recall the literature that tells us those patients living in the 'city' area (intervention group) would be re-hospitalized more frequently than those living in the 'rural' area (control group) ${ }^{10.32}$ In this study the opposite was found. The cost benefit of less hospital admissions observed in our intervention group is therefore more likely to be an underestimation of the real effect.

Lastly, costs for home nursing were gathered differently for the intervention group (prospectively) than for the control group (retrospectively). The costs for home help in the control group had to be estimated (See Appendix), whereas in the intervention group they were gathered prospectively. As was discussed in the results paragraph, this may have biased the observed differences in home nursing and home help costs, resulting in an overestimation of the real effect. Subsequently an alternative method was applied in estimating community nursing and home help costs in the control group (See Results paragraph). Using this method the average total cost was calculated at $15,710 \mathrm{Dfl}$. per patient in the control group. So, one can safely assume that the actual real average total health care costs per patient in the control group patients will lie somewhere between 13,052 and $15,710 \mathrm{Dfl}$.

Assuming that the second and third criticisms are valid, these could only have resulted in an increase of the costs for the intervention group and reduced costs for the control group. We are therefore confident with regard to our final result, i.e. that the intervention did certainly not result in increased total health care costs made by these patients.

Our costs analysis was performed from the health care perspective. Ideally, however, this would have been from the societal perspective. ${ }^{25}$ As our patients were terminally ill, the productivity loss of patients in both groups may be regarded as being the same. Unfortunately, we have no data on the productivity loss of the patients' direct caregivers, nor about the extra costs incurred by the patients being at home for a longer period of their terminal phase. No definite conclusions can therefore be drawn from this study about total costs from a societal perspective. The possible extra costs to patients' direct caregivers were however compensated by a better quality of life as was shown in a previous study. ${ }^{23}$ 
The cost savings on re-hospitalizations seen in our study correspond with those shown by other studies. ${ }^{12.13 .15 .16 .18}$ The greatest savings were made in the last phase of illness. This period is consistent with the time frame over which our patients were studied. The mean survival of our patients was 85 and 68 days for the intervention and control group respectively. Kane et al. ${ }^{14}$ however, did not find any cost savings for rehospitalization in their randomized controlled trial of hospice care. This may be explained by their intervention 'only providing limited help and advice for the patient and his familial caregiver during this time' (i.e. the time the patient was at home).

Although the observed differences in costs for community nursing care and home help in this study may represent an overestimation of the real effect, the increased costs for these areas of care in the intervention programme seem logical when one considers the reduced re-hospitalization of these terminally ill patients. This has also been observed by others. ${ }^{17,33}$

The observed cost reduction for drugs in the intervention group (i.e. those drugs prescribed by the patient's physician and refunded by insurance companies. Those drugs prescribed during an hospital admission were excluded from this category, falling into the category 'costs for hospital admission') may be explained by a more comprehensive prescribing of drugs by physicians, which in turn may have resulted from better coordination of care in this group.

\section{Practice implications}

In summary, our transmural home care programme significantly reduces drug and rehospitalization costs while increasing standard community nursing care and home help costs, when compared to the standard care available in The Netherlands. However, on the whole there is no significant difference in the total sum of health care costs. In view of this, and that the study has previously shown that the intervention contributed significantly to a better quality of life of patients and their direct caregivers, ${ }^{22.23}$ the implementation of a transmural home care programme for terminal cancer patients is recommended for all hospitals with a large multidisciplinary oncology unit. 


\section{References}

1. Macdonald $N$. The interface between oncology and palliative medicine. In: Doyle $D$, Hanks GWC, MacDonald N, eds. Oxford textbook of palliative medicine. Oxford: Oxford University Press, 1997;11-7.

2. Emanuel EJ. Cost savings at the end of life. What do the data show? JAMA 1996;275:1907-14.

3. Riley G, Lubitz J, Prihoda R, Rabey E. The use and costs of Medicare services by cause of death. Inquiry 1987;24:233-44.

4. Johansen H, Nair C, Bond J. Who goes to the hospital? An investigation of high users of hospital days. Health Rep 1994;6:253-77.

5. van den Akker PAM, Tits MHL, Kok NM. Leven met de dood. Over terminale patienten en terminale zorg in Nederland [Living with death. Terminal patients and terminal care in The Netherlands]. Houten: Bohn Stafleu Van Loghum, 1994.

6. Field D, James N. Where and how people die. In: Clark D, ed. The future of palliative care. Buckingham: Open University Press, 1993;6-29.

7. Jones RV, Hansford J, Fiske J. Death from cancer at home: the carers' perspective. BMJ $1993 ; 306: 249-51$.

8. O'Hare PA, Yost LS, McCorkle R. Strategies to improve continuity of care and decrease rehospitalization of cancer patients: a review. Cancer Invest 1993;11:140-58.

9. Wilkes E. Dying now. Lancet 1984;i:950-2.

10. Herd EB. Terminal care in a semi-rural area. Br J Gen Pract 1990;40:248-51.

11. Conkling VK. Continuity of care issues for cancer patients and families. Cancer 1989;64:290-4.

12. Brooks $\mathrm{CH}$, Smyth-Staruch $\mathrm{K}$. Hospice home care cost savings to third-party insurers. Med Care 1984;22:691-703.

13. Mor V, Kidder D. Cost savings in hospice: final results of the National Hospice Study. Health Services Research 1985;20:407-22.

14. Kane LK, Wales J, Bernstein L, Leibowitz A, Kaplan S. A randomised controlled trial of hospice care. Lancet $1984 ; \mathrm{i}: 890-4$.

15. Kidder D. The effects of hospice coverage on Medicare expenditures. Health Services Research 1992;27:195-217. 
16. National Hospice Organization. An analysis of the cost savings of the Medicare Hospice Benefit. Miami: Fla: Lewin-VHI Inc. 1995.

17. McCusker J, Stoddard AM. Effects of an expanding home care program for the terminally ill. Med Care 1987;25:373-85.

18. Raftery JP, Addington-Hall JM, MacDonald LD, Anderson HR, Bland JM, Chamberlain J, et al. A randomized controlled trial of the cost-effectiveness of a district co-ordinating service for terminally ill cancer patients. Palliat Med 1996;10:151-61.

19. Emanuel EJ, Emanuel LL. The economics of dying. The illusion of cost savings at the end of life. $N$ Engl J Med 1994;330:540-4.

20. Nationale Raad voor de Volksgezondheid/College voor Ziekenhuisvoorzieningen. Transmurale somatische zorg [Transmural somatic care]. Zoetermeer: Nationale Raad voor de Volksgezondheid, 1995.

21. Commissie modernisering curatieve zorg. Gedeelde zorg; betere zorg [Shared care; better care]. Zoetermeer: Commissie Modernisering Curatieve Zorg, 1994.

22. Smeenk FWJM, de Witte LP, van Haastregt JCM, Schipper RM, Biezemans JPH, Crebolder HFJM. Transmural care. A new approach in the care for terminal cancer patients: Its effects on re-hospitalization and quality of life. Patient Educ Couns 1997; accepted.

23. Smeenk FWJM, de Witte LP, van Haastregt JCM, Schipper RM, Biezemans JPH, Crebolder HFJM. Transmural care of terminal cancer patients: Effects on the quality of life of direct caregivers. Nurs Res 1997; accepted.

24. Schrijvers AJP. Health and Health Care in the Netherlands. A critical Self-assessment by Dutch Experts in the Medical and health Sciences. Utrecht: De Tijdstroom B.V. 1997.

25. Drummond MF, O'Brien B, Stoddart GL, Torrance GW. Methods for the economic evaluation of health care programmes. Oxford: Oxford University Press, 1997.

26. de Witte LP. After the rehabilitation centre. A study into the course of functioning after discharge from rehabilitation [Thesis]. Amsterdam/Lisse: Swets \& Zeitlinger, 1991.

27. Armitage P, Berry G. Statistical methods in clinical research. Oxford: Blackwell scientific publications, 1987.

28. Tabachnick BG, Fidell CS. Using multivariate statistics. New York: HarperCollins College Publishers, 1996.

29. McWhinney IR, Bass MJ, Orr V. Factors associated with location of death (home or hospital) of patients referred to a palliative care team. Can Med Assoc J 1995;152:361-7. 
Chapter 7

30. Costantini MH, Carnoirano E, Maddedu L, Bruzzi P, Verganelli E, Henriquet F. Palliative home care and place of death among cancer patients: a population-based study. Palliat Med $1993 ; 7: 323-31$.

31. Catalan-Fernandez JGAMAGL, Pons-Sureda O, Recober-Martinez A, Avella-Mestre A, Carbonero-Malberti JM, Benito-Oliver E, et al. Dying of cancer. The place of death and family circumstances. Med Care 1991;29:841-52.

32. Moinpour CM, Pollisar L. Factors affecting place of death of hospice and non-hospice cancer patients. Am J Public Health 1989;79:1549-51.

33. Gray D, MacAdam D, Boldy D. A comparative cost analysis of terminal cancer care in home hospice patients and controls. J Chronic Dis 1987;40:801-10. 


\section{Appendix}

\section{Description of the cost calculations for the different cost categories:}

\section{The costs of the intervention itself:}

1.a. The costs of the Specialist Nurse Coordinator.

All the time spent by this functionary in consultation with the patient, his/her family, the time needed for deliberation with the various caregivers involved with the patient's care, the paperwork et cetera was registered prospectively. This was multiplied by the hourly rate of pay. The regular hourly rate of pay was adjusted to incorporate 'unproductive working hours' (i.e. that proportion of an employee's total number of working hours where he/she is not productive (e.g. coffee breaks etc.) and 'overhead' costs (i.e. for the building, catering, computer costs etc). For the unproductive working hours the generally accepted figure of $15 \%$ was used, and thus the hourly rate of pay was increased by 8 Dutch Guilders (Dfl.) and 4 cents. The cost for 'overheads' was calculated to be 19.83 Dfl. per working hour and was also included in this hourly rate of pay. This adjusted hourly rate of pay was thus calculated at $81.48 \mathrm{Dfl}$. in 1994.

1.b. The costs of the 24-hour Consultation Telephone Service.

The same procedure as described in 1.a. was used. The hourly rate of pay for the functionaries manning this service was calculated at $64.48 \mathrm{Dfl}$. (costs for unproductive working hours 6.34 Dfl. and costs for overheads 15.69 Dfl. per working hour).

1.c. The costs of the Transmural Home Team.

Again the same procedure as described in 1.a. was followed. The hourly rate of pay for this functionary was calculated to be $94.70 \mathrm{Dfl}$. (costs for unproductive working hours 9.35 Dfl. and for overheads $23.05 \mathrm{Dfl}$. per working hour). This total is higher because it also covered 'on call' hours (i.e. those hours the functionary is not actually working but must be available in case his/her help is required/requested by a patient at home).

\section{The costs made by the insurance companies.}

These costs were divided into 6 sub-categories.

2.a. The costs for physician's fees.

2.b. The costs for hospital admissions.

2.c. The costs for outpatient/day care treatment (in the hospital).

2.d. The costs for physiotherapy.

2.e. The costs for prescribed drugs.

2.f. The costs for transportation (ambulance or taxi to and from hospital) and community medical aid supplies (special diet, bandages, et cetera)

Because the hospital charges per day of admission (in 1994 this ranged from 685.80 to 1,022.00 Dfl, depending on the patient's insurance) did not represent the real costs made by these patients, in our analyses we confined ourselves to the real costs incurred by these patients in hospital (i.e. 492.70 Dfl.). All costs made by the hospital for the patient are included in this costing e.g. drugs, catering, laundry, building, heating et 
cetera.

We assumed that the costs the insurance companies made by items 2.a, and 2.c-f represented the approximate real costs of these sub-categories.

3. The costs made by the home care facilities.

This was divided into 3 sub-categories:

3.a. The costs made by 'intensive' (night) community nursing care.

These costs were also covered by the insurance companies and could be retrieved from their databases.

3.b. The costs made for 'standard' community nursing care.

3.c. The costs made for home help

In the intervention group the time the community nurses and home help (family aid) functionaries spent with the patients was registered prospectively. This total time was multiplied by the functionaries' hourly rate of pay, in which the unproductive working time and 'overhead' costs were also included. The hourly rate of pay for the community nurses was calculated at $63.00 \mathrm{Dfl}$. (costs for unproductive working hours $5.60 \mathrm{Dfl}$. and for overhead 10.10 Dfl. per working hour) and that of the home help functionary 40.00 Dfl. (costs for unproductive working hours $4.55 \mathrm{Dfl}$. and for overhead $5.15 \mathrm{Dfl}$. per working hour).

The time spent with patients in the control group was, unfortunately, not registered prospectively. This data had to be gathered retrospectively from the various home care agencies in our area. These agencies were only able to reproduce the time community nurses spent with these patients, not for the home help functionaries. Therefore, the time these home help functionaries had spent with these patients had to be estimated. In doing so, it was assumed that the ratio of 'community nursing time' to 'home help time' would be the same for the intervention and control group. Knowledge of the time spent with the patient by community nurses and home helpers in the intervention and by community nurses in the control group, made an estimation of the time spent by the home help with the patients in the control group possible. 


\section{CHAPTER}

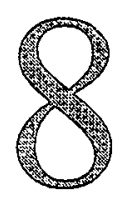

Final considerations 
Chapter 8

\begin{abstract}
In this chapter the main results of this study will be summarized. Some important methodological considerations will be discussed. Recommendations on the health care structure for terminal cancer patients (and other potential patient categories with chronic/complex health problems) are made. These recommendations will be based on the results of this thesis. Finally, suggestions for further research and professional training are put forward.
\end{abstract}




\section{Main results}

Patients with terminal cancer appear, in contrast to what most of them wish, ${ }^{1-3}$ to be frequently re-hospitalized. The main reasons for these re-hospitalizations are the inability to adequately palliate symptoms at home, the overburdening of informal caregivers, and a lack in coordination/continuity of care. ${ }^{4-9}$ To overcome these problems, home care programmes for terminal cancer patients have been developed in several countries. The effects of these home care programmes on the patients' quality of life and their rehospitalization were investigated in a systematic review of the literature (Chapter 2). A widespread search of several data bases on medical literature only revealed 9 studies describing the results of controlled investigations on this subject. Their methodological quality was moderate (median rating 62 on a 100 point scale). Significant positive results were found in 2 out of the 5 studies measuring satisfaction with care, in 3 out of the 7 studies measuring the physical - and in 1 out of the 6 studies measuring psychosocial dimensions of quality of life, and in 2 out of the 5 studies measuring re-hospitalization. Most studies that enabled team members to visit patients at home and/or that included regular team meetings, showed positive results. Considering these results it was concluded that decisive studies were still lacking.

In the Eindhoven region, an attempt was made to improve the supportive care for these terminally ill cancer patients by setting up an intervention programme. This programme was developed in the spirit of transmural care, ${ }^{10}$ in collaboration with the Catharina Hospital Eindhoven, and all the involved local primary and community health care organizations. This transmural home care intervention programme consisted of 4 main elements: a specialist nurse coordinator, a 24-hour telephone consultation service connected to a transmural home team, a home care dossier, and specific care protocols. All these elements intended to improve the coordination of care and thereby the continuity of care provided to these patients as well. This in turn may have positive consequences for both the re-hospitalization of patients, as well as their quality of life and that of their direct caregivers (i.e. that informal caregiver who was considered to be the patient's principal caregiver). These results could be achieved without incurring extra health care costs as a reduction in the costs for hospitalization may balance the extra costs made for the intervention and home care. The extra home care costs is based on the expectation that the intervention would result in less re-hospitalization. These research questions were investigated in this quasi-experimental study and the results are presented in the Chapters 3-7.

Terminally ill cancer patients living in Eindhoven were included in the intervention group (in total 79 patients) and those residing in the surrounding urbanized areas of Eindhoven in the control group (in total 37 patients). Patients in both groups received the standard care available in The Netherlands. ${ }^{11}$ Patients in the intervention group were offered the intervention programme as well. All patients were followed until death and their direct caregivers until 3 months after the patient's death.

In Chapter 3 the workload for professional caregivers and burden for the patients' 
direct caregiver, as well as caregiver satisfaction with the intervention programme, are analysed. This showed that the specialist nurse coordinator needed on average 7.5 hours on each patient case. The 24-hour telephone consultation service was contacted 100 times in total for the 79 patients in the intervention group. The transmural home team only needed to make 8 home visits to patients with problems. On average the patient's general practitioner visited the patient once a week. 'Intensive' community nursing care was necessary in $50 \%$ of cases. The average time spent per patient needing this kind of care was 40 minutes per day. The average daily time needed for standard community nursing was 44 minutes, and for home help 90 minutes. Patients and their various caregivers were (very) satisfied with the programme. The specialist nurse coordinator, and the 24-hour telephone consultation service were considered essential components of the intervention. It was concluded that transmural home care for terminal cancer patients seems feasible given its acceptable workload for the various caregivers.

The effects of the intervention programme on patient re-hospitalization, the patients' quality of life and their place of death are discussed in Chapter 4. Patient re-hospitalization was found to be significantly reduced (from 11.5 to 5.8 days during the terminal phase of their disease; $p<.01$ ), while at the same time significantly contributing to the patients' 'physical' functioning 4 weeks after starting the intervention. 'Psycho-social functioning' was not significantly influenced by the intervention. Furthermore, a higher percentage of patients in the intervention group died at home. This difference was almost significant ( 81 vs $67 \%$ of the patients; $p=.06$ ).

In Chapter 5 the effects of the intervention on the quality of life of the patients' direct caregivers are presented. Because patients were re-hospitalized less with transmural care, the care burden to direct caregivers in the intervention group may have been increased. ${ }^{2}$ This analysis revealed that, despite this possible increase in care burden, the direct caregiver's 'overall quality of life index' significantly improved 1 week after starting the intervention as well as 3 months after the patient had died.

The possible effects of the intervention on indicators of coordination of care and continuity of care are addressed in Chapter 6 . This study found the level of coordination of care, as indicated by task agreement among caregivers, to be poor. The various pairs of caregivers in the intervention group agreed only slightly more on care tasks (3.9 out of 10 tasks) than those in the control group (3.7). However, this poor agreement over care tasks did not seem to create care deficits as the disagreement was hardly ever such that every caregiver thought someone else would perform that specific task. The only significant differences found between the intervention and control group were that 'consultant specialist - specialist nurse coordinator' and 'hospital nurse community nurse' couples in the intervention group showed greater overall agreement. With regard to continuity of care, this study found that communication among caregivers, viewed as an indicator of continuity of care, was already quite adequate in both groups. This left little room for any improvement on this indicator of continuity of care by the intervention. The only significant improvement we found in this area, was that consultant specialists felt that other caregivers in the intervention group were better 
informed about patients. When considering satisfaction with care as an indicator for continuity of care, it was found to be high for both patients and their direct caregivers in both groups (no significant differences between the groups were found). In general it was concluded that this study showed slight positive effects of transmural care on the various indicators of coordination of care and continuity of care. That only slight positive effects were observed is an unexpected result when one considers the positive results the intervention had on re-hospitalization and patient - as well as direct caregiver quality of life. These positive results suggest an improvement in the care process and more specifically the coordination and continuity of care. Several explanations have been put forward for this unexpected result. First, the results on communication for the control group were already very high, thereby limiting the possibility of the intervention creating measurable 'positive' differences. Second, the way in which outcomes were measured (retrospective by means of pre-structured questionnaires) may have been too insensitive to detect possible changes. Furthermore, in this study communication was measured only in a quantitative and not in a qualitative manner. It may well be so, that especially the qualitative aspect of communication was improved by the intervention. This may be expected as the intervention programme enables caregivers to become better informed on the patient's actual health status. Lastly, observation biases, such as 'please your doctor' effects, subjects giving socially desirable answers, as well as contamination biases, may also partly explain these results. Thus, the mechanisms on how transmural care 'works' remain to be elucidated by further (qualitative) studies.

Finally, in Chapter 7, the effects of the programme when compared to standard care on the total health care costs were investigated. This analysis revealed significantly lower pharmaceutical (on average 579 vs. 957 Dutch Guilders [Dfl.] per patient; $p<$ .05 ) and re-hospitalization costs (on average 2,611 vs. 6,257 Dfl. per patient; $p<.01$ ) in the intervention group, whilst community nursing (on average 1,351 vs. $206 \mathrm{Dfl}$. per patient; $p<.001$ ) and home help costs (on average 3,911 vs. 770 Dfl. per patient; $p<$ .01) were significantly higher. No significant difference could be found for the total health care costs (on average 14,323 per patient in the intervention and 13,070 Dfl. per patient in the control group; $p=1.00$ ) between both groups. So, this study showed that transmural home care can substitute hospital care with community care without incurring extra costs.

In view of the overall results presented in Chapters 3-7, it may be concluded that:

- Transmural care of terminal cancer patients in the Dutch health care setting was feasible.

- It significantly reduced re-hospitalization of these patients.

- It contributed positively to the patients' quality of life as measured by 'physical functioning' 1 month after the start of the intervention.

- The patients' direct caregivers' quality of life, as measured by the 'overall quality of life index', was positively influenced by the intervention 1 week after starting the programme as well as 3 months after the patient's death. 
- The intervention had slight positive effects on the indicators of coordination and continuity of care, as applied in this study.

- The programme did not increase the health care cost of caring for these patients.

Thus, our study hypothesis as presented in Chapter 1, can now largely be affirmed (Figure 1).

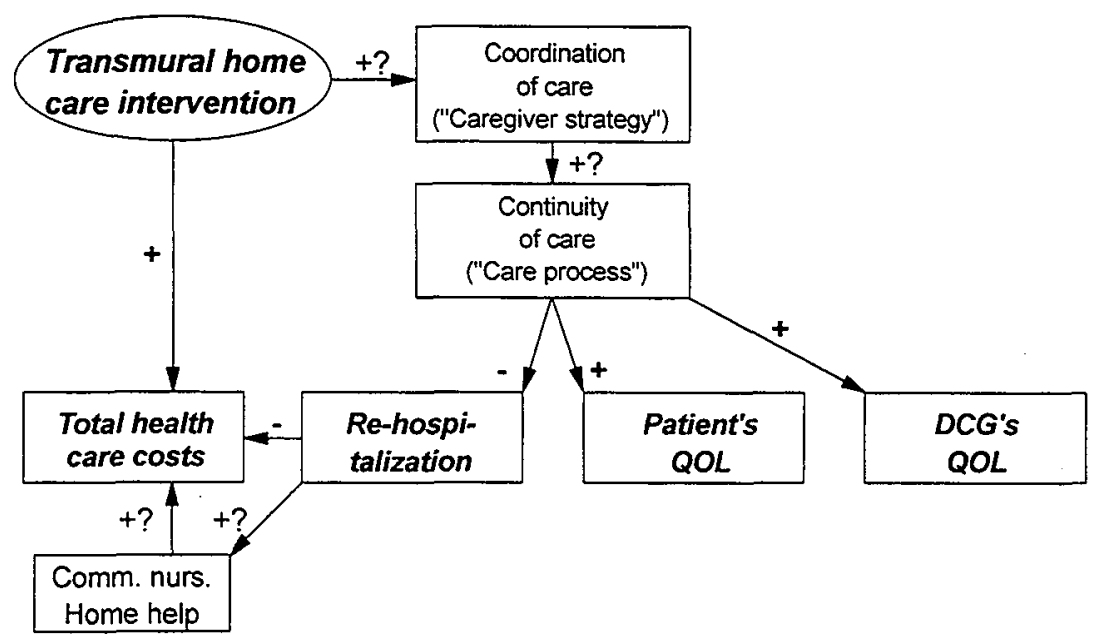

Figure 1 Final study results

$+=$ improves or increases, $-=$ decreases, $Q O L=$ quality of life, $D C G=$ direct caregiver, Comm. nurs. $=$ community nurse.

\section{Methodological considerations}

Today randomised (placebo) controlled trials are considered by many as the sole valid study design for testing the clinical effectiveness of interventions. ${ }^{13}$ This design is relatively easy to conduct in some studies, for example those investigating the effects of pharmaceutical interventions. In others, such as health care intervention studies, randomization may prove impossible because a 'placebo' intervention can in no way be introduced into the control group. The inability to blind patients and investigators to the intervention can pose another restriction..$^{14,15}$ Ethical or practical reasons may also hinder the introduction of a randomized design. ${ }^{15-17}$ Therefore, in those health care intervention studies where randomization proves impossible, other designs, such as quasi-experimental or randomised consent designs, are recommended. ${ }^{14-19}$ Although these kinds of designs may enable investigators to study interventions in this field of medicine, the main disadvantages of the designs (i.e. the chance of study bias influencing the final results) are greatly enhanced compared to randomized controlled trials. ${ }^{16,17,20}$

The study presented in this thesis made use of a quasi-experimental design because 
of unsolvable ethical and practical reasons. In view of the fact that patients living in the city of Eindhoven were allocated to the intervention group, and patients living in the surrounding urbanized areas to the control group, several biases may have theoretically influenced our results. Possible biases (of which the first two mentioned below are also valid for randomised controlled trials) which have to be accounted for are: ${ }^{14,16,17,20}$

- A selection bias because of eligible patients refusing to participate in the study, causing a final study group that is not representative of the group of patients on which the study intends to draw conclusions ('external validity' of the study).

- A selection bias because patients who agreed to participate in the study dropped-out of the study before it terminated.

- Confounding factors: Patient characteristics which may be of influence on the outcome variable of the study. If these characteristics are more prominent in one trial arm, they may result in an observed differences in outcome measures. With regard to this aspect, the different residential areas of the patients included in the study may form a confounding factor.

- A contamination bias because patients in one trial arm may have unintentionally received a treatment/intervention meant for the other trial arm.

- An observation bias because it may have been impossible to blind an intervention from patients and/or investigators.

These possible biases are discussed in the following paragraphs.

First: Could the results be influenced because of a selection bias created by a substantial percentage of patients refusing to participate in the study?

In other words, was the study group representative of the terminal cancer patient population? This question refers to the external validity of the study. In order to be able to answer this, a closer look was taken of all 153 patients asked to participate in this study (for the intervention group [IG] 95 and for the control group [CG] 58 patients). Of these patients, 37 could not be included in the study because they died before the intervention started (in the IG 15 and in the CG 12 patients). Only 1 patient eligible for the intervention and 9 patients eligible for the control group refused to participate in the study. As the refusal rate in the intervention group was very low and the final group of patients studied compared fairly well with those from the control group, it may be concluded that the higher refusal rate for the control group would probably not have lead to a selection bias at this level.

Furthermore, when considering the mean survival of our patients (101 days for the patients in the intervention group and 69 days for the patients in the control group), it may be concluded that our patients were indeed in the terminal phase of their disease. ${ }^{21,22}$ Only 2 patients in the intervention group survived considerably longer than was expected, considering their diagnosis. Even if these patients were discarded from the analyses, our final results remain unchanged.

Lastly, in view of the mortality rate due to cancer in the region, and the adherent population of the Catharina Hospital (See Chapter 1), a calculated maximum of 385 new 
terminal cancer patients could have been expected in the region during the study period. However, not all these patients would have necessarily been admitted to our hospital during the last 6 months of their life. Therefore, the actual number of patients eligible for the study must have been somewhere between 153 (the total number of patients that were asked to participate) and 385. Taking into account the low accrue of patients during the first period (due to treating physicians being insufficiently aware of the study), the final total of 153 patients being asked to participate in the study seems quite satisfactory.

\section{Second: Could a selection bias due to drop-out have influenced our final results?}

Because the drop-out rate in both groups was not significantly different, and the dropout patients matched the final study group population on initial patient characteristics, it is unlikely drop-out would have influenced our findings.

Third: Could, due to the quasi-experimental design of the study, confounding factors be responsible for the observed effects in the various outcome measures instead of the applied intervention programme?

In anticipation of this problem, we collected a broad spectrum of initial patient characteristics at the start of the study, and searched extensively for possible confounders. This data covered the patients' personal, disease and social network characteristics. Potentially confounding factors were included into our final regression analysis. It may then be concluded that our final results (i.e. a positive influence of the intervention programme upon re-hospitalization, the patients' and their direct caregivers' quality of life, and at least no extra health care costs incurred) are valid. Furthermore, the list of initial patient characteristics was so extensive that it seems unlikely that other possible confounders were missed.

Finally, it should be recognized that the different residential areas of the patients from the intervention and control group could have biased the final results. However, because the surrounding area of Eindhoven is highly urbanized and the accessibility to hospital (distance to the hospital has never been a major obstacle for either group of patients), and availability of home care services were equal in both groups, it is highly unlikely that these differences in residency could have posed a major confounder in this study.

\section{Fourth: Could our results be influenced by a possible contamination bias?}

As was observed in Chapter 5 the specialist nurse coordinator also paid some attention to patients in the control group. It should be appreciated that in this research setting, where terminal cancer patients form the subject under investigation, it was not always possible to withhold intervention facilities from all patients in the control group. This may have caused some contamination bias in this study. If that were the case, the observed results in this study would be an underestimation of the real effect rather than the opposite. 


\section{Fifth: Could an observation bias have influenced our final results?}

In order to minimize the chance of this happening, we tried to keep patients from the intervention group separated from those in the control group as much as possible. So patients in the control group were unaware of what patients receiving the intervention programme experienced. In order to prevent an observation bias by the 2 interviewers collecting data on the quality of life outcome measures, both were blinded to the study design. Furthermore, they were trained to interview these patients, used structured questionnaires and were in no way involved with the investigating group.

Despite these precautions an observation bias at patient and/or interviewer level cannot be completely excluded. The interviewers, by nature of their contact with the patients, could well have gained an idea of the actual study design. Also, patients in the intervention group were offered extra facilities (these extra facilities alone and the accompanying attention may have helped the patient feel better) whereas patients in the control group only received the standard care available. If such an observation bias was present, the greatest influence would be expected early on in the study (i.e. at T2 as patient enthusiasm about the study would be at its greatest) and positive results would more likely be found in the psychological dimensions of quality of life (as this observation bias in particular would be caused by the patient's subjective feelings). Because this study found positive results in the patients' 'physical' functioning (and not in their 'psychological' functioning) at $\mathrm{T} 3$ (and not at $\mathrm{T} 2$ ) and on their rate of re-hospitalization, it may be concluded that an observation bias is most unlikely to have caused the observed results. This problem of observation bias, however, will remain difficult to solve in these kinds of studies, because it is virtually impossible to offer patients in the control group a placebo intervention.

Some additional considerations may be made regarding the patient's quality of life analysis.

Firstly, one has to realize that the outcome measures used for the patient's quality of life analysis in this study, i.e 'physical' and 'psychosocial functioning', have a broader meaning than physical and psychosocial functioning in its strictest sense. In this study 'physical functioning' comprises the outcome scores: 'pain', 'somatic complaints', and 'Sickness Impact Profile'. 'Psychosocial functioning' comprises the outcomes scores: 'fear', 'loneliness', 'general well-being', and 'psychological complaints'.

Secondly, it may be argued that the number of patients who could be evaluated in the quality of life analysis was relatively small. Because regression analysis may become instable where low numbers of subjects are included, the positive effect found on the patient's 'physical functioning' by the intervention should be considered with some caution.

\section{Implications and recommendations}

A high percentage of patients in The Netherlands have multiple chronic health problems 
needing continuous care from both primary - and hospital based caregivers. ${ }^{23}$ Terminal cancer patients in particular belong to this group of patients. Because in The Netherlands, as in most countries, no regular facility exists in the present health care structure for the coordination of care for these patients, it is often inadequate, ${ }^{24.25}$ resulting in a fragmented health care. ${ }^{26}$ This in turn may jeopardize the continuity of care for these patients. ${ }^{6.26}$ We tried to improve coordination, and thereby continuity of care for these terminal cancer patients by setting up a transmural home care programme based in the hospital. The ultimate goal was to tailor the care needed by these patients in such a way that it would meet their individual needs. Every effort should be made to deliver care as an uninterrupted, continuous process, and wherever possible in the patient's home. It should be noted that the programme had no intentions of the hospital domineering the care of patients at home. On the contrary, it aimed to support the primary care team in retaining these patients within their own homes for as long as possible.

Considering the results of this thesis, some recommendations can be put forward regarding the way care should be organized for terminal cancer patients being simultaneously cared for by primary and hospital care teams. While giving these recommendations, it has also to be acknowledged that the patients studied in this thesis needed a great deal of hospital care. Under these circumstances it seems beneficial to organize the care provided for these patients as follows:

- The responsibility for the coordination of care should belong to that caregiver system of most importance for the patients, i.e. for terminal cancer patients the hospital is of most importance because patients may frequently need care from their consultant specialists in order to adequately palliate symptoms. For the coordination of tasks the hospital has to appoint a specialized functionary who: coordinates the discharge of these patients from hospital, arranges and tailors the care needed by patients at home to meet their individual needs, to monitor the care provision process, and to track down and solve possible shortcomings.

- In order to support the primary care team in caring for these patients in the community and to improve the continuity of care, a 24-hour telephone consultation service should be installed in the hospital. If problems arise at home, primary caregivers, patients and their informal caregivers should be able to contact this service for advice without obstacles (as in this programme, without interference of a telephone operator). In order to be able to give adequate advice, the staff manning this service should have knowledge of the patient's disease (as here, where a copy of the patient's home care dossier is available). For medical questions it should be possible to contact a consultant specialist immediately.

- If high technology treatment modalities (e.g. epidural pain analgesia or intravenous medication) is supplied to patients at home, the hospital should retain responsibility for the service in case problems arise with these applications. This requires the installation of a transmural home team being able to give a 24 hour service.

- Coordination of care among caregivers should be further improved by the implementation of a patient dossier containing all essential medical and nursing informa- 
tion. This dossier should be used by - and easily accessible for all caregivers involved. In this programme this dossier was kept at the patient's home. Considering today's communication possibilities, an electronic dossier accessible for all caregivers involved may also be an alternative. ${ }^{27}$ This (electronic) dossier, however, cannot be implemented without special precautions to maintain patient privacy. Legislation in The Netherlands, as in most other countries, requires medical information to be kept inaccessible to unauthorized parties (i.e. other than the caregivers involved) unless patients give their written consent.

- Specific multidisciplinary care protocols for specialized (high-technological) treatment modalities should be drawn up. These protocols should standardize and support caregivers when using specific skills and actions. By drawing up these protocols multidisciplinary coordination of care will be systematically improved.

- As this study showed that task agreement among caregivers (which may be considered as an indicator for the level of coordination of care) was poor, appropriate measures should be taken in order to enhance this. In our view measures for this should be taken at an administrative, health care policy level as well as at a local operational level.

At the administrative, health care policy level we advise the discussion of this problem in a meeting on this topic involving all the various professional caregiver organizations. The goal of this meeting should be to make an inventory of the various tasks which may have to be performed for these patients and to reach a consensus on which tasks should be assigned to which particular professional caregiver.

At the local operational level a possible solution to this task agreement problem would be for the care coordinator to make an inventory of the tasks that should be performed on a particular patient, and assign these to the various appropriate caregivers at the beginning of the care process. The coordinator will have to notify the various caregivers, and anticipate potential future misunderstandings. This should be noted in the patient's dossier.

- Where the level of intensity of basic nursing cannot be sufficiently met by the informal and professional caregivers, the integration of a palliative care unit within a nursing home would meet this need. If the patient was cared for on such a unit, it would in no way hinder the use of the programmes beneficial facilities.

By implementing these recommendations the quality of care (considering the positive results of our intervention programme on re-hospitalization and most probably on the patients' and their direct caregivers' quality of life too) provided to terminally ill cancer patients will improve. These recommendations may also be of benefit for the quality of care provided to patients with other complex and chronic health care problems. A particularly positive element to these recommendations may be that they do not interfere with the existing health care structure in The Netherlands. Thereby the chance of a successful implementation of such a programme is considerably enhanced.

In view of our experiences from this study, other important prerequisites for the 
successful implementation of such programmes are:

- All (professional) health care organizations should be involved in the development of the programme from the very start, and all should approve of the final version of the programme.

- The professional role of each caregiver in the care process should be fully acknowledged by all.

- The possible 'extra' care burden to caregivers should be acceptable.

- Appropriate incentives for each caregiver involved should be made available by the responsible health care politicians and health care insurance companies.

This last prerequisite may represent an important barrier for the further implementation of this kind of transmural care. The Dutch health care system is financed in such a way that hospitals, consultant specialists, general practitioners, and home care services all have separate financing structures with fixed budgets. This system therefore contains practically no financial incentives for transmural care. For instance, general practitioners, who receive a capitated fee for most patients are often better off referring complex and chronically ill patients to hospitals. Hospitals may generate empty beds by providing transmural care, which may lead to a downward adjustments of their budgets. Lastly, home care agencies already complaining about their too tight and fixed budgets, will not be receptive to having to provide care for a new group of patients.

One way of solving this incentive problem, without substantially transforming the Dutch health care financing system, would be to award hospitals budgets that accommodate the costs they would have to make for those patients in the standard care situation. These budgets would have to be fixed for several years. In this situation hospitals may then be able to benefit from programmes that will reduce re-hospitalization of patients. On the other hand, however, these programmes would probably generate an additional workload for other (primary care based) caregivers. As it would be in the hospitals' interest for these programmes to succeed, they are more likely to agree to recompense this extra workload for the primary care teams from their own budgets.

In our region, the first steps on this trajectory have already been undertaken by the adjustment of a fixed budget for the hospital (within the framework of the so-called 'Zorgvernieuwing en Bekostiging Ziekenhuiszorg' [Care Innovation and Financing Hospital Care] project in our region), by the regional health care insurance companies.

If such transmural care programmes are to be implemented on larger scales this may also have several implications for the education and training programmes of physicians (general practitioners as well as consultant specialists), hospital - and community nurses.

First, it may be advisable to improve the general practitioners' knowledge of today's (high technology) palliative treatment possibilities, and medical specialists' knowledge of the (high technological) treatment possibilities for patients at home. Practical courses in the community for physicians training to become consultant specialist, and practical courses in hospitals for physicians training to be general practitioners, may be set up. This last item has recently been incorporated into the Dutch training programme for general practitioners. 
Second, nurse training institutions should establish a post-graduate course for the function of 'specialist nurse coordinator' (also known as 'transfer nurse') because, at present, no course exists for this functionary in The Netherlands.

Third, as with consultant specialists and general practitioners, training programmes for hospital nurses and community nurses should incorporate courses that enable them to gain knowledge about each others professions and care capacity. Furthermore, during the 'community nursing' course, adequate attention should be paid to the various 'hightechnology' intervention techniques now available to patients at home.

\section{Future research}

Although this thesis answered some of the questions related to the effects of transmural care, many others remain unanswered. Future research should be directed at these unresolved items:

- What is the effect of transmural care, compared to standard care, on the workload of community nurses and home helpers?

Our study only gave some insight into this aspect. In Chapter 7 it was shown that costs for community nursing and home help were substantially higher for patients in the intervention group. This suggests that the care burden for these caregivers would be heightened by the transmural care intervention programme. However, because costs for community nursing and home help were gathered prospectively in the intervention group, whereas costs for community nursing were retrieved retrospectively and costs for home help had to be estimated in the control group, it was suspected that the observed differences on these costs between the 2 groups would more likely to be an overestimation of the real difference. Therefore, no definite conclusions may be drawn on this from our results.

- What is the effect of transmural care on costs when observed from the societal perspective?

This study revealed that the total health care costs for patients in the intervention group were, at least, no higher when compared to those costs made for patients receiving standard care. Because costs for community nursing in the control group had to be gathered retrospectively and costs for home help in this group had to be estimated, the difference observed between the intervention and control group may have been an overestimation of the real difference. If so, transmural care may well turn out to be cheaper than standard care when considering health care costs alone. On the other hand, costs should ideally have been looked at from the societal perspective. ${ }^{28}$ In our study this turned out to be impossible. An important cost aspect which may thereby have been missed in our study is the income loss of informal caregivers. Although it will be difficult to monitor all costs (which will be necessary when performing an economic health care analysis from the societal perspective), future research on this aspect should never- 
theless be aimed at comparing costs from a societal perspective.

- By what mechanism does transmural care cause its positive effects both on rehospitalization as well as the patients' and their direct caregivers' quality of life? This study failed to answer this question. This may have been due to the use of inappropriate outcome parameters. Future research on this area will have to be prospective and make use of appropriate outcome parameters for coordination and continuity of care (quantitative as well as qualitative).

- What is the effect of transmural care in other patient categories with chronic and complex health problems, e.g. chronic obstructive pulmonary disease, congestive heart failure and diabetes mellitus?

In case quality of life is also considered an outcome measure in these studies, from our experience (where many patients dropped-out because of overburdening by the extensive quality of life questionnaires we used), we recommend the use of a disease specific and well validated quality of life questionnaire, which can be filled in by patients within 15-30 minutes.

- What are the effects of intervention programmes aimed at improving task agreement among caregivers, on the patient's quality of life, re-hospitalization, costs, patient and caregiver satisfaction?

As our study showed that task agreement among caregivers was poor, an intervention aimed at improving this may be beneficial to patients. 


\section{References}

1. McWhinney IR, Bass MJ, Orr V. Factors associated with location of death (home or hospital) of patients referred to a palliative care team. Can Med Assoc J 1995;152:361-7.

2. Brown D, Roberts JA, Elkins TE, Larson D, Hopkins M. Hard choices: the gynecologic cancer patient's end-of-life preferences. Gynecol Oncol 1994;55:355-62.

3. Townsend J, Frank AO, Fermont D, Dyer S, Karron O, Wallgrove A, et al. Terminal cancer care and patients' preference for place of death: a prospective study. BMJ 1990;301:415-7.

4. Jones RV, Hansford J, Fiske J. Death from cancer at home: the carers' perspective. $B M J$ 1993;306:249-51.

5. Wilkes E. Dying now. Lancet 1984;i:950-2.

6. O'Hare PA, Yost LS, McCorkle R. Strategies to improve continuity of care and decrease rehospitalization of cancer patients: a review. Cancer Invest 1993;11:140-58.

7. Conkling VK. Continuity of care issues for cancer patients and families. Cancer 1989;64:290-4.

8. Herd EB. Terminal care in a semi-rural area. Br J Gen Pract 1990;40:248-51.

9. Johansen $\mathrm{H}$, Nair C, Bond J. Who goes to the hospital? An investigation of high users of hospital days. Health Rep 1994;6:253-77.

10. Kruyt J, Slingerland P. Transmurale zorg in de praktijk. Tussen thuis en ziekenhuis [Transmural care in practice. Between home and hospital]. Utrecht: NIZW Uitgeverij, 1996.

11. Schrijvers AJP. Health and Health Care in the Netherlands. A critical Self-assessment by Dutch Experts in the Medical and health Sciences. Utrecht: De Tijdstroom B.V. 1997.

12. McMillan SC. Quality of life of primary caregivers of hospice patients with cancer. Cancer Pract 1996;4:191-8.

13. McQuay H, Moore A. Need for rigorous assessment of palliative care. Although difficult, randomised controlled trials are mandatory. BMJ 1994;309:1315-6.

14. Knottnerus JA. Gezondheidszorg onderzoek in extramurale settings [Health care research in an extra-mural setting]. In: ten Have HAMJ, Engberts DP, Kalkman-Bogerd LE, Kimsma GK, Jensma-Nieuwpoort ACB, eds. Ethiek en recht in de gezondheidszorg. Deventer: Kluwer, 1995;151-93.

15. Knottnerus JA, Dinant GJ. Medicine based evidence, a prerequisite for evidence based medicine. Future research methods must find ways of accommodating clinical reality, not 
Chapter 8

ignoring it. $B M J$ 1997;315:1109-10.

16. Polit DF, Hungler BP. Nursing research. Principles and methods. Philadelphia: J.B. Lippincott company, 1995.

17. Cook TD, Campbell DT. Quasi-experimentation. Design \& analysis issues for field settings. Boston: Houghton Mifflin Company, 1979.

18. Black N. Why we need observational studies to evaluate the effectiveness of health care. $B M J 1996 ; 312: 1215-8$.

19. McWhinney IR, Bass MJ, Donner A. Evaluation of a palliative care service: problems and pitfalls. $B M J 1994 ; 309: 1340-2$.

20. Tabachnick BG, Fidell CS. Using multivariate statistics. New York: HarperCollins College Publishers, 1996.

21. Reuben DB, Mor V, Hiris J. Clinical symptoms and length of survival in patients with terminal cancer. Arch Intern Med 1988;148:1586-91.

22. Wachtel T, Allen-Masterson S, Reuben D, Goldberg R, Mor V. The end stage cancer patient: terminal common pathway. Hosp $J$ 1988;4:43-80.

23. Knottnerus JA, Metsemakers J, Hoppener P, Limonard C. Chronic Illness in the community and the concept of 'social prevalence'. Fam Pract 1992;9:15-21.

24. Courtens AM. Kenmerken van zorg en kwaliteit van leven bij patienten met kanker [Characteristics of care and quality of life in cancer patients; Thesis]. Maastricht: Universitaire Pers Maastricht, 1993.

25. de Witte LP. After the rehabilitation centre. A study into the course of functioning after discharge from rehabilitation [Thesis]. Amsterdam/Lisse: Swets \& Zeitlinger, 1991.

26. Philipsen $\mathrm{H}$, Stevens FCJ. Modernization, rationality, and continuity of care: Theoretical concepts and empirical findings. Sociological Focus 1997;30:189-204.

27. Hampson JP, Roberts RI, Morgan DA. Shared care: a review of the literature. Fam Pract 1996;13:264-79.

28. Drummond MF, O'Brien B, Stoddart GL, Torrance GW. Methods for the economic evaluation of health care programmes. Oxford: Oxford University Press, 1997. 


\section{CHAPTER}

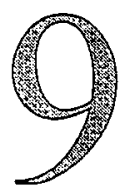

Summary 
The purpose of this thesis was to investigate the effects of a transmural home care programme for terminal cancer patients.

In Chapter 1 an introduction to the care problem for terminal cancer patients is given. Despite the wishes of most cancer patients to spend as much time as possible in their own intimate homes, these patients appear to be re-hospitalized frequently and often die in hospital as well. Reasons for these re-admissions are the inability to palliate symptoms at home, overburdening of direct caregivers (i.e. that informal caregiver thought to be the patient's principal caregiver), and poor coordination/continuity of care.

We tried to overcome these problems by introducing a transmural home care programme for these patients. The main elements of this intervention were: a specialist nurse coordinator, a 24-hour telephone consultation service in the hospital connected to a transmural home team, a home care dossier, and specific care protocols. All these elements intended to improve the coordination of care provided for these patients, and in doing so, hopefully improve the continuity of care as well. By improving the coordination and continuity of care it was hypothesized that this programme, compared to standard care, would lower the re-hospitalization of patients, without negative effects on either the patients' or their direct caregivers' quality of life, nor on the total health care costs.

Due to ethical and practical reasons the study design had to be quasi-experimental (non-randomized controlled). Patients meeting the inclusion criteria (incurable cancer, estimated prognosis of less than 6 months, older than 18 years, fully informed of their disease, willing to sign an informed consent, being admitted to the multidisciplinary oncology ward of our hospital) and living in Eindhoven were allocated to the intervention group. The other patients living in the urbanized surroundings of Eindhoven were allocated to the control group. The control group received the standard care available in The Netherlands. This includes care from their general practitioner, community nurse, home help, meals on wheals and so on. If necessary, community nursing can be provided around the clock ('intensive' community nursing). All patients were followed until death and their direct caregivers until 3 months after the patient's death.

In Chapter 2 the results of a systematic review of the literature on the effects of home care programmes for terminal cancer patients upon their quality of life and rehospitalization, are presented. In total, only 9 prospective controlled studies were identified which could be included in our analysis. Their methodological quality was moderate. The effects of these programmes on the patients' quality of life and their re-hospitalization were inconsistent. There was a tendency for those programmes which included team members able to visit patients at their homes, and hold regular team meetings, to show positive results. It was concluded, that in view of these findings and the huge problem of terminal cancer care to society, further properly designed studies were urgently needed.

Chapter 3 describes the care process and workload the intervention programme imposed upon the various caregivers. Patient and caregiver satisfaction with the programme was also examined. The mean survival time of the 79 patients included in 
the intervention group was 101.2 days, of which an average of 5.8 days were spent in hospital. For her care coordinating tasks the specialist nurse coordinator spent an average of 7.5 hours for each patient. The 24-hour telephone service was contacted 100 times in total during the whole care process, and the transmural home team was asked to visit patients in their homes on only 8 occasions. On average the patient's general practitioner visited the patient about once a week. The average daily time needed for community nursing and home help was 44 and 90 minutes respectively. Patients and their various caregivers judged the intervention programme (very) positively.

In Chapter 4 the results of the intervention on the patients' re-hospitalization, place of death and quality of life is presented. The patients in the intervention group (79 patients) underwent significantly less re-hospitalization (5.8 vs. 11.5 days), compared to the control group (37 patients) during the terminal phase of their illness. The intervention also had a significantly positive effect on the patients' 'physical functioning' 4 weeks after their discharge from hospital (discharge being the starting point of the intervention). The patients' 'psycho-social functioning', however, was not significantly influenced by the intervention programme. In the intervention group $80.8 \%$ of the patients died at home. This percentage was $64.9 \%$ in the control group. This difference was nearly significant $(p=.06)$

The effects of the intervention upon the patients' direct caregivers' quality of life is demonstrated in Chapter 5. Although these direct caregivers had to take care of patients for a greater part of his/her remaining life, the intervention appeared to have a significantly positive effect on the caregivers' 'overall quality of life index' 1 week after the patient was discharged from hospital and 3 months after the patient had died. Four weeks after the patients' discharge, the same tendency was observed. However, this did not reach statistical significance, possibly due to the low number of direct caregivers who could be analysed at that time point.

Chapter 6 describes the effects of the transmural intervention programme on indicators of coordination of care and continuity of care. Slight improvements were found on the indicators of coordination and continuity of care applied in this study.

The level of task agreement among caregivers, considered an indicator of coordination of care, was poor. The various couples of caregivers in the intervention group showed little more agreement about care tasks (3.9 out of 10 tasks) than those in the control group (3.7). This poor agreement on care tasks, however, did not seem to lead to care deficits. The disagreement was hardly ever such that every caregiver thought someone else would have to perform that specific task. The only significant differences found between the intervention and control group were that 'consultant specialist specialist nurse coordinator' and 'hospital nurse - community nurse' couples in the intervention group showed greater overall agreement.

With regard to communication, being considered an indicator of continuity of care, this study found that communication among caregivers in both groups was already most adequate. This left little room for any improvement by the intervention on this indicator of continuity of care. The only significant improvement we found was that the consultant 
specialists were of the opinion that other caregivers in the intervention group were better informed over the patients.

When considering satisfaction with care as an indicator of continuity of care, this was high for both patients and their direct caregivers in both groups (no significant differences between the groups).

Several methodological reasons were put forward to explain why only slight effects on indicators of coordination and continuity of care were observed. It was concluded that future prospective and qualitative studies will be needed to further elucidate the possible effects of transmural care on coordination and continuity of care.

Whether the intervention would induce extra health care costs was the aim of the study presented in Chapter 7. In this analysis real costs rather than costs refunded by insurance companies were considered as much as possible. The intervention was shown to significantly lower costs for drugs and hospital admissions. On the other hand it increased the costs for standard community nursing and home help. Because costs for community nursing and home help were collected prospectively in the intervention group, whereas in the control group community nursing costs had to be retrieved retrospectively and home help had to be estimated (as these were not filed by the various agencies), we speculated that this observed difference might have been an overestimation of the real difference. Nevertheless, the average total health care costs made for each patient in the intervention group was not significantly different from that in the control group. The average total costs for the intervention itself were 598 Dutch guilders per patient.

In Chapter 8 we have summarized our main results and discussed some of the methodological problems of a quasi-experimental intervention study. The main conclusions of this study were as follows:

- This transmural home care programme for terminal cancer patients significantly reduces patient hospitalization (cf. most patients wishes);

- The programme most probably has a positive effect on the patients' and their direct caregivers' quality of life; and

- The programme does not incur extra health care costs.

Therefore, we recommend that such programmes should be installed in every hospital and related primary care teams dealing with terminal cancer patients. Recommendations for setting up such programmes are given as well as for future scientific research and professional training. 


\section{CHAPTER}

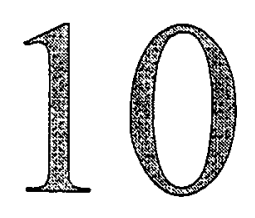

\section{Samenvatting}


Dit proefschrift beschrijft de resultaten van onderzoek naar de effecten van een transmuraal thuiszorgprogramma voor terminale kankerpatiënten.

In Hoofdstuk 1 wordt een overzicht gegeven van (zorg-)problemen die zich bij terminale kankerpatiënten kunnen voordoen. Hoewel de meeste patiënten het liefst zo lang mogelijk in hun eigen vertrouwde thuissituatie verzorgd willen worden en indien mogelijk ook thuis willen sterven, worden ze toch vaak ge(re-)hospitaliseerd en overlijden zij in een ziekenhuis en niet thuis. Redenen voor opname van deze patiënten in een ziekenhuis zijn: (1) onvoldoende mogelijkheden in de thuissituatie om de klachten van patiënten adequaat te palliëren, (2) overbelasting van de informele mantelzorg, en/of (3) inadequate coördinatie/continuïteit van zorg.

Door de implementatie van een transmuraal thuiszorgprogramma voor deze patiënten hebben we getracht deze problemen op te lossen. De belangrijkste elementen van dit interventieprogramma waren:

(1) een thuiszorgcoördinator, die het ontslag van patiënten uit het ziekenhuis voorbereidde en die zorg voor de patiënt thuis regelde die hij/zij nodig had,

(2) een 24-uurssteunpunt gekoppeld aan een transmuraal team,

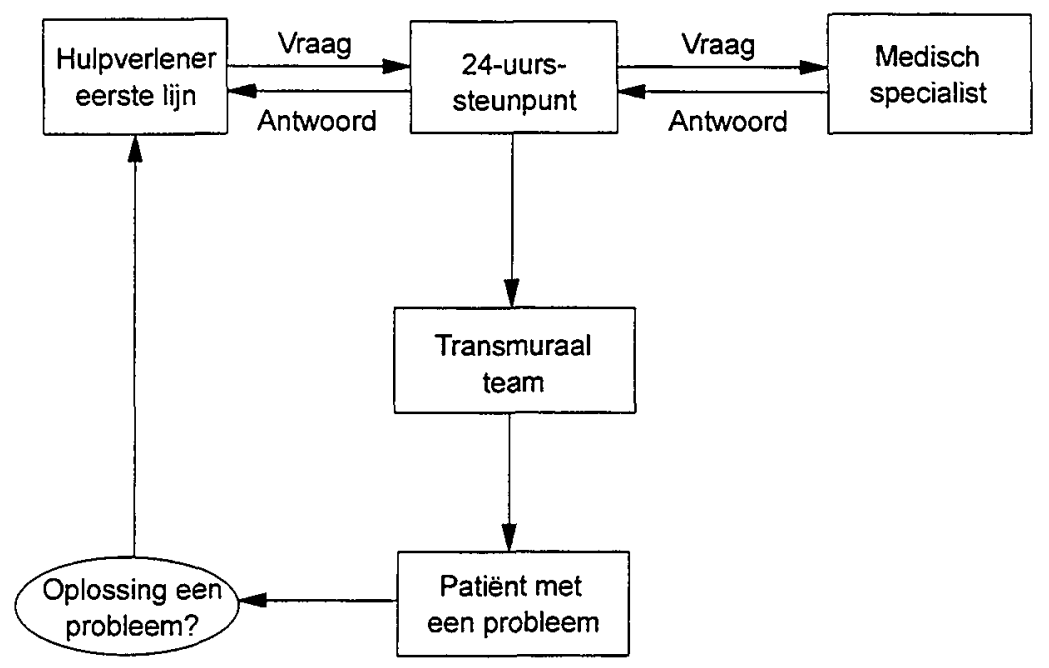

\section{Organisatiestructuur van het 24-uurssteunpunt en transmuraal team}

Bij zorgproblemen in de eerste lijn konden hulpverleners uit deze eerste lijn eventueel contact opnemen met het 24-uurssteunpunt. Bij verpleegkundig technische problemen konden ziekenhuisverpleegkundigen van het transmurale team hulpverlening thuis bieden.

(3) een thuiszorgdossier, waarin alle essentiële medische, verpleegkundige en overige zorggegevens waren opgenomen, en

(4) zorginhoudelijke protocollen (bijvoorbeeld protocollen met betrekking tot infuusbehandeling of epidurale pijnbestrijding thuis). 
Al deze elementen hadden tot doel de coördinatie van zorg en daardoor ook de continuïteit van zorg voor de patiënten te verbeteren. Er werd verondersteld dat dit interventieprogramma de coördinatie en de continuïteit van zorg zou verbeteren. Hierdoor zou, vergeleken met de reguliere zorg, de re-hospitalisatie van de patiënten kunnen verminderen, zonder dat dit negatieve effecten zou hebben voor de kwaliteit van leven van deze patiënten en hun belangrijkste informele verzorgers. Omdat verwacht werd dat de kosten voor hospitalisatie zouden dalen, maar dat de kosten voor thuiszorg door het programma zouden toenemen, werd aangenomen dat de totale kosten voor de gezondheidszorg niet zouden toenemen.

Om ethische en praktische redenen bleek alleen een quasi-experimentele (nietgerandomiseerde, gecontroleerde) onderzoeksopzet mogelijk te zijn. Patiënten die voldeden aan de inclusiecriteria (niet meer te genezen vorm van kanker, geschatte levensduur van 6 maanden of minder, ouder dan 18 jaar, volledig geïnformeerd over de diagnose, bereid een 'informed consent' te tekenen en opgenomen op de multidisciplinaire afdeling Oncologie van het Catharina Ziekenhuis Eindhoven) en die woonachtig waren in Eindhoven werden geïncludeerd in de interventiegroep. De patiënten die woonachtig waren in de verstedelijkte omgeving van Eindhoven werden geïncludeerd in de controlegroep. Deze patiënten kregen de gebruikelijke reguliere zorg (inclusief de wijkverpleging, gezinszorg, terminale thuiszorg, et cetera). Alle patiënten werden gevolgd tot hun overlijden. De belangrijkste informele verzorgers werden gevolgd tot 3 maanden na het overlijden van de patiënt.

In Hoofdstuk 2 worden de resultaten van literatuuronderzoek betreffende de effectiviteit van thuiszorgprogramma's voor terminale kankerpatiënten met betrekking tot rehospitalisatie en kwaliteit van leven besproken. In totaal werden slechts negen prospectieve gecontroleerde studies gevonden, die geïncludeerd konden worden in de analyses. De methodologische kwaliteit van deze studies bleek matig. De effecten die de, in deze onderzoeken beschreven, programma's hadden op de re-hospitalisatie en de kwaliteit van leven van de patiënten bleken niet eenduidig te zijn. De programma's waarbij het voor de teamleden van de thuiszorgprogramma's mogelijk gemaakt was om patiënten thuis te bezoeken en/of die regelmatig multidisciplinaire teambesprekingen hadden geïncorporeerd in hun programma, bleken vaker positieve effecten te vertonen dan situaties met reguliere zorg. Gezien deze bevindingen en de omvang van het zorgprobleem voor terminale kankerpatiënten, besluit dit hoofdstuk met de conclusie dat verder goed opgezet onderzoek naar de effecten van deze thuiszorgprogramma's dringend noodzakelijk is.

Hoofdstuk 3 beschrijft het zorgproces en de werkbelasting voor de verschillende professionele zorgverleners in het transmurale thuiszorgprogramma. De tevredenheid van de patiënten en hun zorgverleners werd tevens onderzocht. Van 1 januari 1994 tot 1 februari 1995 werden 79 patiënten geïncludeerd in het transmurale thuiszorgprogramma. De gemiddelde overleving van deze patiënten was 101 dagen, waarvan zij gemiddeld 5,8 dagen in een ziekenhuis waren opgenomen. De thuiszorgcoördinator besteedde gemiddeld 7,5 uren per patiënt aan haar coördinerende taken. Gedurende het gehele 
zorgproces namen patiënten en hun zorgverleners honderd maal contact op met het 24uurssteunpunt en heeft het transmurale team acht huisbezoeken bij patiënten afgelegd in verband met verpleegkundig technische problemen. De huisarts bleek gemiddeld één huisbezoek per week bij deze patiënten af te leggen, terwijl wijkverpleegkundigen en gezinshulpen de patiënt gedurende dagelijks gemiddeld respectievelijk 44 minuten en 90 minuten hulp boden. De patiënten en hun verschillende verzorgers beoordeelden het thuiszorgprogramma over het algemeen als (erg) positief.

In Hoofdstuk 4 worden de effecten van het thuiszorgprogramma op re-hospitalisatie, plaats van overlijden en kwaliteit van leven van de patiënten gepresenteerd. De 79 patiënten in de interventiegroep bleken gedurende de rest van hun leven gemiddeld significant minder gehospitaliseerd te zijn dan de 37 patiënten in de controlegroep (5.8 vs. 11.5 dagen). De interventie droeg vier weken na ontslag van de patiënten uit het ziekenhuis (het moment waarop het transmurale thuiszorgprogramma kon beginnen) ook significant bij tot het 'fysiek functioneren' van deze patiënten. Het 'psycho-sociaal functioneren' van de patiënten bleek echter niet significant beïnvloed te worden door de interventie. In de interventiegroep bleek bijna 81 procent van de patiënten thuis te overlijden. In de controlegroep was dit percentage 65 . Dit verschil bleek net niet significant te zijn $(p=.06)$.

De effecten van de interventie op de kwaliteit van leven van de belangrijkste informele verzorger van de patiënt worden besproken in Hoofdstuk 5 . Hoewel deze verzorgers de patiënten van de interventiegroep gedurende een langere tijd verzorgden, bleek dat de interventie positief bijdroeg aan de 'overall kwaliteit van leven' van deze verzorgers één week nadat de patiënt uit het ziekenhuis was ontslagen en drie maanden nadat de patiënt was overleden. Dezelfde trend werd gezien vier weken na ontslag van de patiënt uit het ziekenhuis.

Hoofdstuk 6 beschrijft de effecten van het transmurale thuiszorgprogramma op indicatoren van coördinatie en continuïteit van zorg. In deze studie werden slechts geringe verbeteringen gevonden. Het niveau van de taakovereenstemming tussen de verschillende zorgverleners, één van de indicatoren van coördinatie van zorg, was gering. De verschillende koppels zorgverleners in de interventiegroep bleken gemiddeld over iets meer zorgtaken overeenstemming te hebben dan die in de controlegroep (overeenstemming over 3,9 versus 3,7 zorgtaken uit een set van 10 zorgtaken). Deze geringe overeenstemming leidde zelden tot hiaten in de uitvoering hiervan, aangezien de onenigheid met betrekking tot het verrichten van de diverse zorgtaken zelden zodanig was dat de zorgverleners van mening waren dat anderen dan zijzelf deze specifieke taak zouden moeten verrichten. Het enige significante verschil dat ten aanzien van de taakovereenstemming gevonden werd, was dat de koppels 'specialist - thuiszorgcoördinator' en 'ziekenhuisverpleegkundige - wijkverpleegkundige' in de interventiegroep gemiddeld een hogere mate van taakovereenkomst aangaven dan deze koppels in de controlegroep.

De communicatie, een indicator van de continuïteit van zorg, bleek optimaal tussen de betrokken zorgverleners in de beide groepen. De potentiële bijdrage van de interventie was hierdoor beperkt. De enige significante verbetering die op dit gebied gevonden 
werd, was dat de ziekenhuisspecialisten van mening waren dat de andere zorgverleners in de interventiegroep, vergeleken met die uit de controlegroep, beter geïnformeerd waren over de omstandigheden van de patiënt.

De 'tevredenheid over de zorg', een tweede indicator voor de continuilteit van zorg, was hoog bij zowel de patiënten als hun verzorgers in beide groepen. Tussen de beide groepen werden geen significante verschillen gevonden.

In dit hoofdstuk zijn verscheidene methodologische overwegingen besproken ter explicatie van de bevinding dat slechts geringe effecten gevonden werden ten aanzien van coördinatie en continuïteit van zorg. Geconcludeerd werd dat nadere prospectief opgezette studies en ook kwalitatieve onderzoeken gewenst zijn om de mogelijke effecten van transmurale zorg op coördinatie en continuïteit van zorg te registreren.

Hoofdstuk 7 behandelt de effecten van de interventie op de kosten van de gezondheidszorg. In de analyse werd uitgegaan van de werkelijk gemaakte kosten en niet van de kosten zoals deze vergoed werden door de ziektekostenverzekeraars. De kosten voor medicamenten en voor ziekenhuisopnames waren in de interventiegroep significant lager. De kosten voor wijkverpleging en gezinshulp waren in deze groep echter significant hoger. Omdat de kosten voor wijkverpleging en gezinshulp in de interventiegroep prospectief waren gemeten, terwijl de kosten voor wijkverpleging in de controlegroep retrospectief waren verzameld en de kosten voor gezinshulp in deze groep zelfs geschat moesten worden (omdat de verschillende thuiszorgbureau's deze gezinshulp in de onderzoeksperiode niet geregistreerd hadden), hebben we verondersteld dat het gevonden verschil in kosten voor de wijkverpleging en gezinszorg waarschijnlijk een overschatting is van het werkelijke verschil. Niettegenstaande deze mogelijke overschatting, werd er geen significant verschil gevonden in de gemiddelde totale kosten voor de gezondheidszorg voor de patiënten uit de interventiegroep en de controlegroep.

In Hoofdstuk 8 worden de gevonden resultaten samengevat en worden de methodologische problemen van een quasi-experimenteel onderzoek bediscussieerd. De belangrijkste conclusies die getrokken konden worden waren:

- Het, in dit proefschrift beschreven, transmurale zorgprogramma voor terminale kankerpatiënten vermindert de hospitalisatie van deze patiënten (conform de wens van de meeste patiënten);

- Het programma beïnvloedt waarschijnlijk de kwaliteit van leven van deze patiënten en hun belangrijkste informele verzorgers positief; en

- Het programma leidt niet tot een verhoging van de kosten van de gezondheidszorg.

Daarom wordt geadviseerd dergelijke programma's te implementeren in ieder ziekenhuis en de daaraan gerelateerde complementaire eerstelijnsgezondheidszorg. Aanbevelingen voor het opzetten van dergelijke programma's worden gegeven en tevens voor verder wetenschappelijk onderzoek en onderwijs. 


\section{Dankwoord}

Allereerst dient in dit dankwoord vermeld te worden dat dit proefschrift niet had kunnen verschijnen zonder de bereidwillige medewerking van alle patiënten en hun informele verzorgers, die aan dit onderzoek hebben deelgenomen. Hoewel zij de moeilijkste periode in hun leven doormaakten, waren zij toch bereid aan dit onderzoek mee te doen, alle vragenlijsten in te vullen en hun medewerking te verlenen aan verschillende interviews. Bijzonder veel dank ben ik dan ook aan hen verschuldigd.

Dat de totstandkoming van dit proefschrift in meerdere opzichten de resultante is van een 'transmuraal' project, moge tevens blijken uit de lijst van overige personen en instanties, allen van groot belang bij de voltooiing ervan. Mijn dank gaat naar hen allen uit.

- Prof.dr. H.F.J.M. Crebolder, promotor. Beste Harry, de wijze waarop jij mij hebt begeleid was zeer inspirerend. De snelheid waarmee jij telkens weer de verschillende conceptartikelen kon doomemen en de wijze waarop jij daarbij telkens weer de puntjes op de ' $i$ ' kon zetten, bewonder ik zeer. Onze 'thuiszorgvergaderingen', die meestal bij jou thuis plaatsvonden, begin ik nu al een beetje te missen. Ik hoop dat we deze bijzonder prettige samenwerking in de toekomst verder kunnen uitbouwen.

- Dr. L.P. de Witte, co-promotor. Beste Luc, jij doorzag vanaf het begin het belang van dit onderzoek en liet je niet uit het veld slaan door het quasi-experimentele design ervan. Integendeel, jij zag het als een uitdaging om, rekening houdend met de 'handicaps' van het design, de effecten van dit transmurale thuiszorgprogramma toch op een wetenschappelijk verantwoorde wijze te analyseren. Veel heb ik daarvan geleerd. Hopelijk kunnen ook wij deze vruchtbare samenwerking, welke in alle opzichten zeer plezierig is geweest, in de toekomst verder voortzetten.

- De leden van de beoordelingscommissie, prof.dr. C. Spreeuwenberg, dr. J.P.M. Diederiks, prof.dr. H.F.P. Hillen, prof.dr. E. Schadé en prof.dr. A.J.P. Schrijvers dank ik voor de aandacht die zij aan dit proefschrift besteed hebben en hun opbouwende kritiek, waarvan ik dankbaar gebruik gemaakt heb.

- De projectgroep 'Thuiszorg voor de Kankerpatiënt'. In deze projectgroep namen deel vanuit de eerste lijn vertegenwoordigers van de District Huisartsen Vereniging (mevr.drs. A. Lucas en drs. P. Bertholet), de Stichting Thuiszorg Eindhoven (mevr. J. van der Wel), de 'stadsapothekers' (drs. P.P.J. Batenburg) en de Stichting Vrijwilligers Thuiszorg (mevr. M. Vonck $\dagger$ ). De tweede lijn was vertegenwoordigd middels vertegenwoordigers van de medische staf (drs. R.M. Schipper, voorzitter van de projectgroep, dr. W. Breed, en dr. H.H.M. Korsten), de ziekenhuisapotheek 
(mevr.drs. T. Veenbaas), de ziekenhuisverpleegkundigen (de heer J. van Gassel), de thuiszorgcoördinator (mevr. E.M.A.C. Gubbels), de secretaresse Oncologie (mevr. C. Vogels), de projectmanager (P. Leijten) en de wetenschappelijk begeleider van het project (drs. J.P.H. Biezemans). Tot slot was het Intergraal Kankercentrum Zuid middels mevr. T. van der Pol vertegenwoordigd in de projectgroep. Deze projectgroep heeft het zorgmodel ontwikkeld en de implementatie ervan begeleid. Zonder iemand tekort te willen doen zijn bij de implementatie van het project van eminent belang geweest drs. R.M. Schipper (voorzitter van de projectgroep), drs. J.P.H. Biezemans, mevr. E.M.A.C. Gubbels en mevr. J. van der Wel. Beste Rob, Hans, Els en Jopie, zonder jullie inbreng had dit project nooit kunnen slagen. Een speciaal woord van dank dient daarbij uit te gaan naar mijn 'maatje' Rob, die mij enthousiast gemaakt heeft voor de wetenschappelijke uitwerking van dit project. Zonder hem had ik waarschijnlijk nooit de kans gekregen om aan dit onderzoek te beginnen.

- De landelijke begeleidingscommissie. Deze commissie, ingesteld door de Nationale Commissie Chronisch Zieken (NCCZ), heeft als klankbord gefunctioneerd voor de projectgroep. Vooral in het begin heeft de projectgroep vele waardevolle suggesties van de commissie mogen ontvangen.

- Mevr.drs. J.C.M. van Haastregt. Beste Jolanda, een betere 'research assistent' had ik me niet kunnen wensen. Altijd kon ik met vragen bij jou terecht en altijd was je bereid om nog 'even' iets uit te zoeken. Deze lijn is in de eindfase van het onderzoek voortgezet door je opvolgster, mevr.drs. I.W.C.J. Nooyen. Jolanda en Ingrid bedankt voor jullie steun en geduld met mij.

- Dr. A.J.H.A. Ament. Beste André, mede dankzij jou, is een belangrijk onderdeel van dit proefschrift, de kostenanalyse, voltooid. Dank voor jou steun hierbij.

- Mijn 'Maten'. Beste Harm, Kees, Rob, en Jacques, jullie stimuleerden mij en gaven mij daar waar nodig de ruimte om dit proefschrift te voltooien. Het is voor mij een waar voorrecht om deel uit te mogen maken van 'onze' maatschap.

- De interviewers, R. Smeets en mevr. B. de Beer. Zij hebben de interviews met betrekking tot kwaliteit van leven bij de patiënten en hun informele verzorgers afgenomen. De nauwgezetheid en het geduld waarmee zij zich van deze taak gekweten hebben verdient veel waardering.

- Mevr. C. Gubbels (secretaresse van de afdeling pulmonologie) en mevr. C. Vogels (secretaresse afdeling Multi-Disciplinaire Oncologie). Het zal eenieder duidelijk zijn dat het schrijven van dit proefschrift ook veel extra werk heeft opgeleverd voor mijn 'eigen' secretaresse. Beste Carola, altijd als ik weer eens stukken kwijt was, wist jij ze wel weer te vinden en met mijn pietluttigheid bij het verzenden van 
artikelen heb je altijd veel geduld gehad. Mijn dank daarvoor.

Beste Corrie, jij zorgde dat de overdrachten en de diensten van diegenen die bij het project betrokken waren altijd gesmeerd liepen. Zonder jou zou de continuiteit in de zorgverlening zeker schade hebben opgelopen.

- De discussies met dr. G.P.L.A. van den Broek (geneesheer directeur van het Catharina Ziekenhuis) en dr. J.-W.W. Coebergh (epidemioloog, IKZ) met betrekking tot de resultaten van dit proefschrift en de verdere implementatie daarvan waren altijd zeer inspirerend. Beste Frits en Jan-Willem, dank voor jullie altijd opbouwende commentaar.

- G. van Dael. Beste Guy, de lay-out ziet er, zoals gebruikelijk bij jou, weer fantastisch uit.

- Mevr. E. Moors en mevr. M. van Leeuwen, de bibliothecaresses. Beste Els en Marianne, veel dank voor het verzamelen van de door mij benodigde literatuur.

- En verder iedereen die bij dit project betrokken is geweest en zich hiervoor heeft ingezet, zoals de verpleegkundigen van de afdeling MDO en het 'Transmuraal team' hebben bemand, de specialisten, de huisartsen, de wijkverpleegkundigen, de gezinsverzorgers, de vrijwilligers, et cetera.

- S. Cardiff heeft het Engels gecorrigeerd. Dear Shaun, thank you very much indeed for your numerous corrections. These were most helpful and appreciated.

- Mevr.drs. D.M.M. Fleuren, mijn buurvrouw, heeft mijn Nederlands gecorrigeerd. Beste Dorine, zoals je gemerkt hebt is Nederlands nooit mijn beste vak geweest op de middelbare school. Dank voor je correcties.

- Mijn paranimfen, drs. H.Ch. Gooszen en dr.ir. H.A. Fleuren, CFPIM. Beste Harm en Hein, dank dat ik van jullie organisatorische talenten gebruik mocht maken.

- Mijn ouders dank ik voor de gelegenheid die zij mij gaven om te studeren, voor hun liefde, steun en vertrouwen.

- Lieve Anneke, vaak zeg je dat je niets aan dit proefschrift hebt bijgedragen. Ik weet echter wel beter! Zonder jouw onvoorwaardelijke liefde en steun had ik dit proefschrift nooit kunnen afronden.

- Lieve Michiel en Wouter, eindelijk is deze 'kjompoeter' (= computer waarop dit proefschrift geschreven is) dan voor jullie. Lieve Anne Marije, eindelijk kan papa jou ook adequate 'thuiszorg' geven. 


\section{Bibliography}

- Smeenk FWJM, Gooszen HCh, Pieterman H, Jürgens PhJ. The value of MRI compared to computer tomography in the evaluation of intrathoracic lesions [Abstract]. Eur Respir J 1988;1:301s.

- Smeenk FWJM, Gooszen HCh, Pieterman H, Jürgens PhJ. De waarde van MRI van de thorax in vergelijking met de CT bij de evaluatie van ruimte innemende processen in de thorax [Abstract]. Ned Tijdschr Geneeskd 1989;133:324-5.

- Smeenk FWJM, Serlie J, van der Jagt EJ, Postmus PE. Bullous degeneration of the left lower lobe in a heroin addict. Eur Respir J 1990;3:1224-6.

- Smeenk FWJM, Twisk SPM, Berreklouw E, Gooszen HCh, Postmus PE. Dyspnea after pneumonectomy. Eur Respir J 1991;4:243-5.

- Ziora D, Jansveld CAF, Smeenk FWJM. Peak Expiratory Flow (PEF) versus spirometry in patients with airflow obstruction during a single clinical visit. Eur Respir Soc, Wenen, Sept 1992.

- Smeenk FWJM, Postmus PE. Interatrial right to left shunting developing after pulmonary resection in the absence of elevated right sided heart pressures. Chest 1993;10:528-31.

- Jansveld CAF, Smeenk FWJM, Schipper RM, Arends B-J, Lanting A. No relationship between smoking habits and irregular opacities on the chest roentgenogram of 104 patients from general practice [Abstract]. Chest 1993;103:174S.

- Smeenk FWJM, Jongerius CM. Beeldvormende technieken voor de diagnostiek van longziekten. Historisch overzicht vanaf 1913. Pulmoscript 1993;4:37-43.

- Ziora D, Jansveld C, Smeenk $F$. Usefulness of peak expiratory flow measurements in assessing the degree of bronchial obstruction in patients with chronic obstructive lung diseases. Pneumonol Alergol Pol 1994;64:35-40.

- Steenhuis LH, Tjon a Tham RTO, Smeenk FWJM. Hernia van Bochdalek, een zeldzame oorzaak van empyema thoracis [Abstract]. Ned Tijdschr Geneeskd 1994;138:269.

- Steenhuis LH, Tjon aTham RTO, Smeenk FWJM. A rare cause of empyema thoracis. Eur Respir $J$ 1994;7:204-206. 
- Smeenk FWJM, Postmus PE. Interatrial Right-to-Left shunting in the absence of elevated right-sided heart pressures after major trauma. Chest 1994;105:1614.

- Smeenk FWJM, Klinkhamer P, Jansz AJ, Breed W, Jansveld CAF. Opportunistische infecties van de long: Mogelijk een bijwerking van inhalatie corticosteroïden? Ned Tijdschr Geneeskd 1996;104:94-8.

- Smeenk FWJM. De uitvoering van de consensus 'Diagnostiek longembolie' in de praktijk [Letter]. Ned Tijdschr Geneeskd 1996;140:389.

- Smeenk FWJM, Jansen J, van den Bosch M, Schönberger JPAM, Postmus PE. Effects of four different methods of sampling arterial blood and storage time on gas tensions and shunt calculation in the 100\% oxygen test. Eur Respir J 1997;10:9103.

- Smeenk FWJM, Klinkhamer P, Jansz AJ, Breed W, Jansveld CAF. Additional experiences with corticosteroids in COPD [Letter]. Chest 1997;112:1715-6.

- Smeenk FWJM, de Witte LP, Crebolder HFJM. Good terminal care and its importance for informal caregivers. Nurs Res 1998;47:8-9. 


\section{Curriculum vitae}

De auteur is geboren op 10 juni 1958 te Ittersum (destijds gemeente Zwollerkerspel). In 1972 verhuisde hij naar Staphorst. Zijn Atheneum-B-diploma behaalde hij in 1976 aan de Rijksscholengemeenschap te Meppel. In datzelfde jaar begon hij met zijn studie Geneeskunde aan de Rijksuniversiteit te Groningen (RUG). Van 1979 tot 1981 was hij student-assistent bij de Vakgroep Longziekten. Onder leiding van prof.dr. N.G.M. Orie, en later prof.dr. H.J. Sluiter $\dagger$ deed hij onder andere onderzoek naar het 'slaapapneusyndroom'. Aan de RUG behaalde hij in 1981 zijn doctoraalexamen en in 1983 zijn artsexamen. In datzelfde jaar begon hij met zijn basisopleiding Interne Geneeskunde in het ziekenhuis 'De Weezenlanden' te Zwolle (opleider dr. J.A. ten Bokkel Huinink). Op 1 januari 1986 begon hij met de opleiding tot 'Arts voor Longziekten en Tuberculose' in het Catharina Ziekenhuis te Eindhoven (opleider dr. C.A.F. Jansveld). Deze opleiding werd met succes afgesloten op 1 januari 1989. Vanaf die tijd tot 1 mei 1989 was hij 'chef de clinique' in diezelfde kliniek en van 1 mei 1989 tot 1 mei 1990 was hij als longarts verbonden aan het Academisch Ziekenhuis te Groningen. Vanaf 1 mei 1990 is hij opgenomen in de maatschap 'Longziekten' van het Catharina Ziekenhuis te Eindhoven (dr. C.A.F. Jansveld, H.Ch. Gooszen, R.M. Schipper en per 1 januari 1998 J.P.H.M. Creemers). Vanaf 1995 is hij plaatsvervangend opleider.

Het in dit proefschrift beschreven onderzoek 'Transmural care of terminal cancer patients' heeft plaatsgevonden in de 'adherentie' regio van het Catharina Ziekenhuis te Eindhoven. Het thuiszorgprogramma was gestationeerd in dit ziekenhuis. Het onderzoek vond plaats van 1994-1995. Na deze onderzoeksfase is het thuiszorgprogramma volledig geïmplementeerd in het ziekenhuis.

De auteur is getrouwd met Anneke Witziers. Zij hebben drie kinderen: Michiel (8 jaar), Wouter ( 7 jaar) en Anne Marije ( 4 jaar). 